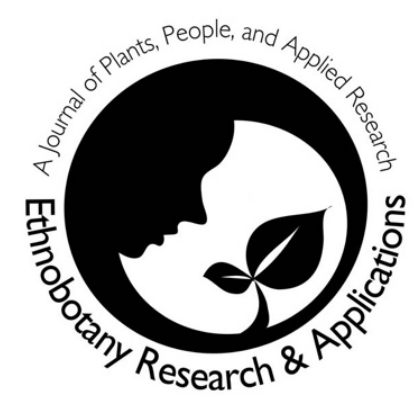

\title{
The subalpine and alpine vegetation of the Georgian Caucasus - a first ethnobotanical and phytosociological synopsis
}

\author{
George Nakhutsrishvili, Ketevan Batsatsashvili, Rainer W. \\ Bussmann, Inayat Ur Rahman, Robbie E. Hart and Shiekh \\ Marifatul Haq
}

\begin{abstract}
Correspondence
George Nakhutsrishvili ${ }^{1}$, Ketevan Batsatsashvili ${ }^{2}$, Rainer W. Bussmann ${ }^{3 *}$, Inayat Ur Rahman ${ }^{4}$, Robbie E. Hart ${ }^{5}$, Shiekh Marifatul Haq ${ }^{6}$

${ }^{1}$ Focal Point, Caucasus Plant RLA, Institute of Botany, Ilia State University, Tbilisi, Georgia, makgeorg@gmail.com ${ }^{2}$ Focal Point, Caucasus Plant RLA, Institute of Botany, Ilia State University, Tbilisi, Georgia, ketevan.batsatsashvili@iliauni.edu.ge

${ }^{3}$ Department of Ethnobotany, Institute of Botany and Bakuriani Alpine Botanical Garden, Ilia State University, Tbilisi, Georgia, rainer.bussmann@iliauni.edu.ge

${ }^{4}$ Department of Botany, Hazara University, Mansehra 21300, KP, Pakistan, hajibotanist@outlook.com

${ }^{5}$ William L. Brown Center, Missouri Botanical Garden, St. Louis, USA, robbie.hart@mobot.org

${ }^{6}$ Wildlife Crime Control Division, Wildlife Trust of India, Noida, India. snaryan17@gmail.com

*Corresponding Author: rainer.bussmann@iliauni.edu.ge
\end{abstract}

Ethnobotany Research and Applications 23:12 (2022)

\section{Research}

\begin{abstract}
Background:The Republic of Georgia is part of the Caucasus biodiversity hotspot, and human agricultural plant use dates back at least 6000 years. Over the last years lots of ethnobotanical research on the area has been published. In this paper we analyze the use of food plants in the $80 \%$ of Georgia not occupied by Russian forces. We hypothesized that, (1) given the long tradition of plant use, and the isolation under Soviet rule, plant use both based on home gardens and wild harvesting would be more pronounced in Georgia than in the wider region, (2) food plant use knowledge would be widely and equally spread in most of Georgia, (3) there would still be incidence of knowledge loss despite wide plant use, especially in climatically favored agricultural regions in Western and Eastern Georgia. The alpine vegetation of the Caucasus hotspot has fascinated botanists for centuries. Given the very complicated biogeographic setting, a concise classification of vegetation communities has however eluded science so far.
\end{abstract}

Methods: The present work, based on 619 plots, is the first study to attempt a concise phytosociological classification. Even given this large number of samples a complete vegetation classification still proved difficult, and more releveés are needed for a detailed assessment, following Braun-Blanquet. It is also the first attempt to give an overview on plant uses in the alpine and subalpine areas of Georgia.

For plant uses we employed the very large dataset that we gathered all over Georgia interviewing over 300 participants from 2014-2019. For the vegetation analysis we employed traditional phytosociological table work combined with an analysis based on species composition, coverage and abiotic factors using "R", compared to a 
classification using "Twinspan". From 2013 to 2019 we also interviewed over 380 participants in all regions of Georgia not occupied by Russian forces. All interviews were carried out in the participants' homes and gardens by native speakers of Georgian and its dialects (Imeretian, Rachian, Lechkhumian, Tush, Khevsurian, Psavian, Kakhetian), other Kartvelian languages (Megrelian, Svan) and minority languages (Ossetian, Ude, Azeri, Armenian, Greek).

Results: We found that 183 species of the subalpine and alpine flora, representing about $33 \%$ of all species encountered in the vegetation survey, had reported uses. The usage of alpine and subalpine species in Georgia is mostly focused on the use of the plants as fodder, which is unsurprising. Green crops such as Lactuca sativa, Phaseolus vulgaris, Ocimum basilicum, Mentha x piperita, Allium cepa, and Artemisia dracunculus are grown virtually everywhere. Cucurbita pepo, Cucumis sativus, Solanum melongena, and Zea mays, all introduced species, were discovered to be popular elements in local cuisine. In human and veterinary medicine, however, Matricaria chamomilla, Berberis vulgaris, and Juniperus hemisphaerica are still used. There are two vegetation classes: Bromopsis variegatae - Festucaetea ovinea (Class. nov.) (Subalpine pastures), which has seven orders and twelve alliances/eighteen associations, and Sympoholoma graveolensis - Saxifragetea exaratae (Class. nov.) (Alpine pastures), which has one order, two alliances, and four associations.

Conclusions: The alpine and subalpine vegetation of the Greater Caucasus and its uses were assessed in detail for the first time, highlighting the still existing gaps in both phytosociological and ethnobotanical work. Given the establishment of borders in post-soviet independence, it will be interesting to see how long this original crosscultural knowledge will remain, given that the actual use of the traditional knowledge, as well as cross-border high altitude pastoralism are declining.

Keywords: Republic of Georgia, Caucasus, Traditional Knowledge, Knowledge loss, Food plants, Conservation, Vegetation analysis, vegetation communities, syntaxonomy

\section{Background}

Natural plant communities are being threatened by environmental changes such as global warming and increased human activity (Malhi et al., 2020). Alpine and subalpine ecosystems with cool climatic conditions are thought to be sensitive to environmental changes (Takahashi, 2018). Georgia's high altitudes are abundant in plant life, with a genetic diversity of medicinal plants, a wild relative of crop plants, and other economically important species. These fragile ecosystems are marked by rareness of topography, climate, soil, vegetation, and ethno-culture (Payal et al., 2020). As a result, a strategy for evaluating the relationship between vegetation and environmental conditions is to analyze plant species and classify them ecologically (Altaf et al., 2021). Traditional data regarding traditional use of natural resources and effective biodiversity protection can be found in ethnobotanical studies (Haq et al., 2021).

Georgia is situated between latitudes $41^{\circ}$ and $44^{\circ} \mathrm{N}$, and longitudes $40^{\circ}$ and $47^{\circ} \mathrm{E}$, with an area of $69700 \mathrm{~km}^{2}$, with $20 \%$ of the country currently occupied by Russian troops. Georgia politically associates with the European Union and takes part in all major programs of European development and cooperation. Georgia can be defined as a transcontinental country on the divide between Asia and Europe, with its larger part located south to this divide (i.e., in Asia) and smaller but strategically important parts (e.g., Khevi, Piriketi Khevsureti,) located north of the continental divide (i.e., in Europe) (Bussmann et al. 2014).

The uplift of the Georgian Caucasus started in the late Oligocene and shares the same structural characteristics as the younger mountains of Europe. The Greater Caucasus mostly includes Cretaceous and Jurassic rocks, interspersed with Paleozoic and Precambrian formations in higher regions. Hard, crystalline, metamorphosed rocks like schist and gneisses, as well as pre-Jurassic granites are found in the western part, while softer, Early and Middle Jurassic clayey schist and sandstones in the eastern part. The foots of the Greater Caucasus are built of younger limestone, sandstones, and marls. The Lesser Caucasus in contrast is predominantly formed of Paleogene rocks interspersed with Jurassic and Cretaceous formations. The youngest geological structures of Georgia are represented by the vast volcanic plateaus in the southern part of the country. These divisions lead to an extremely complex terrain with pronounced climatic gradients: (1) the mountains of the greater Caucasus with peaks over $5000 \mathrm{~m}$ (Shkara, Babis Mta, Chanchakhi, etc.); (2) the inter-mountain plains between the Greater and Lesser Caucasus mountains; (3) the mountains of the Lesser Caucasus with peaks rarely exceeding 3000 m (Mepistskaro, Kheva, Shavi Klde, Kanis Mta, Arsiani); (4) the Volcanic plateau of the Southern Georgia with elevations from 1300-2200 m. (Nakhurtsrishvili 1999, 2021; Zazanashvili et al. 2000). 
Georgia's climate is influenced by its location in the warm temperate zone stretching from the Black to the Caspian Seas, and the complexity of its terrain. Georgia has a coastline of $330 \mathrm{~km}$ with warm climate, the mean temperature reaching $4-7^{\circ} \mathrm{C}$ in January and $22-23^{\circ} \mathrm{C}$ in July, and high precipitation (1500-2000 mm annually). The warm oceanicsubtropical climate can be found only at lower elevations (less than $650 \mathrm{~m}$ ); in more elevated terrains and to the north and east the climate becomes moderately warm. The Greater Caucasus bars cold air from the north, while warm and moist air from the Black Sea spreads easily into the coastal lowlands until reaching the Likhi range, which partly impedes further westward movement of the warm and moist air. In central Georgia precipitation in mountains can be twice that in the plains. In the mountains weather conditions change to cool and wet quite steeply with increasing elevation and above $2100 \mathrm{~m}$ the environment becomes sub-alpine and alpine, with permanent snow and ice above 3600 m (Nakhurtsrishvili 1999, 2021; Zazanashvili et al. 2000).

\section{The alpine and subalpine vegetation of the Caucasus}

High-mountain vegetation (from the viewpoint of botany) comprises the area situated above the climatic limit of trees. They are characterized by specific environmental conditions: high solar radiation, high ratio of ultraviolet radiation, low temperature, and low atmospheric pressure, (Larcher 1980, Körner 2003). The mean temperature of the growth period is generally $6-8{ }^{\circ} \mathrm{C}$ (Körner et al. 2003, Körner and Paulsen 2004, Pauli et al. 2011).One of the most pronounced peculiarities of life above the forest limit is the diversity of micro-habitats across even a very small area (Körner 2004, Nakhutsrishvili et al. 2011, 2012, 2017).

Georgia's climate is determined by its location within a warm temperate zone between the Black and Caspian Seas, and the complexity of its terrain in which mountain ranges and their orientation play an important part. The coastline of Georgia is $330 \mathrm{~km}$ long and the climate of the coastal zone is warm: the mean temperature is $4-7^{\circ} \mathrm{C}$ in January and $22-23^{\circ} \mathrm{C}$ in July. Precipitation is abundant (1500-2000 mm annually), especially in the southern part. At the same time, The Greater Caucasus mountains bars cold air from the north, while warm and moist air from the Black Sea spreads easily into the coastal lowlands from the west till the range of Likhi, which partly impedes further westward movement of the warm and moist air waves. In central Georgia, precipitation in mountains can be twice of that in the plains. Likewise, in the west the warm oceanic-subtropical climate can be found only at lower elevations (less than $650 \mathrm{~m}$ ), in more elevated terrains and also to the north and east the climate becomes moderately warm. In the mountains weather conditions change to cool and wet quite steeply with increasing elevation and above $2100 \mathrm{~m}$ the environment becomes subalpine and alpine, permanent snow and ice are found above $3600 \mathrm{~m}$ (Nakhutsrishvili et al. 2017).

The debate on the origin of the alpine flora of the Caucasus has been going on for over a century. Kuznetsov (1915) hypothesized that the alpine flora of the Caucasus did not develop uniformly over time, and that many alpine plants existed as far back as the Miocene, but the bulk of the alpine flora arrived later from descendants of the forest vegetation. In his opinion, in the glacial periods, the alpine flora of the Caucasus became enriched due to the intensive exchange with the mountains of Europe and Asia. Kuznetsov (1915) dated the alpine flora to the Sarmatian, considering it essentially autochthonous. Medvedev (1915) suggests assemblages that were close to the modern tall herbaceous vegetation or to Rhododendron thickets with ancient Tertiary elements. The modern alpine vegetation, in his opinion, appeared only during the Pleistocene. Fedorov $(1942,1952)$ suggested that the alpine flora falls in three groups: arcto-alpine, alpine Caucasian and alpine Eurasian, of which only the first group is migratory. Maleev (1948) believed that the basis of modern subalpine and alpine meadows mostly established in glacial periods as a result of boreal invasions. Takhtajan (1946) recognized low stature grassland (alpine swards), pteridophytes and chionophile species. In his later work, Takhtajan (1957) suggested that moderate (alpine) and cryophilus (arctic) floras were of low-latitude mountain origin. Kharkevich (1954) advocated a migration hypothesis for the formation of the alpine flora of the Caucasus in the Quaternary Age. Popov (1949) believed that the alpine flora of the Caucasus was autochthonous. Elenevskyi $(1964,1965)$ dated subalpine and alpine meadows of the Lesser Caucasus to Quaternary formations, while subalpine tall herbaceous vegetation as well as Rhododendron thickets and rock vegetation should be dated back to Tertiary formations. Most Caucasian botanists regarded the highmountain flora of the region to consist chiefly of the boreal and arctic-alpine elements, which radiated into this area during the Pleistocene. Later, the most typical representatives of the Caucasian alpine flora were believed to be of autochthonous origin (Fedorov 1952, Kharadze 1960), and that the alpine flora of today is of Tertiary origin, developed during the Quaternary. In his analysis of Caucasian endemism, Grossheim (1936) indicated that the area of the Greater Caucasus was a region for "processing" of migrating boreal and Asia Minor elements (Nakhutsrishvili et al. 2017). 
The most conservative paleoendemic of the Caucasian high mountains have remained in the central parts and at the boundaries of the ice shield. In the Alps and Carpathians, as well as in the Caucasus, the highest mountain ranges served as refugia for the pre-glacial ancient endemic flora (Kharadze 1960). Both progressive and conservative paleoendemic can be found in the highest part of the Caucasian mountains. Conservative paleoendemic are exemplified by Sredinskya grandis, Campanula mirabilis, C. ossetica, Edraianthus owerinianus, Symphyandra lezgina. Progressive paleoendemic species include, Charesia akinfiewii, Petrocoma hoefftiana and Symphyandra pendula (Nakhutsrishvili et al. 2017).

The contribution of Zernov 2006) is the most recent publication that covers the history of the flora of the Caucasus, particularly of the North-West Caucasus. According to this author, most of the species of the alpine flora of the Caucasus were developed from pre-pleistocene migrants. Their penetration into the Caucasus took place partially from the north, partially from the south, from South-West Asia. Agakhanjanz and Breckle 2002) concluded that endemism is strongly influenced by the presence or absence of forest belts and thus the climatic conditions in the migratory belts. These belts are, however, often fragmented by riverbeds, erosion gullies, rock-cliffs, which altogether would permit by passing the forest belts (Nakhutsrishvili et al. 2017).

Kharadze (1960) outlined that the following species are originating from Daghestan, e.g., Stipa daghestanica, Thymus daghestanicus, Heliotropium styligerum. She also suggests that the limestone mountain massifs of the North Caucasus participated in the formation of the pre-Pleistocene xerophytic flora. The example of such mountains is the limestone Rocky Range of the Great Caucasus. Autochthonous hemi xerophytic elements are well represented in the areas of the Central Caucasus from the mid-montane to the subnival belts. Among the distant relations of the Caucasian oreo xerophytic flora, the connections with those of the Mediterranean and Asia Minor should be mentioned. Ancient connections with the Mediterranean flora have been found in the following genera: Astragalus, Nepeta, Scabiosa, Symphyandra, Edraianthus. The Late Pliocene is the period of the most ancient migrations from Asia Minor to the Caucasus. Radiation of these elements into the Caucasus was associated with the xeric periods of the Pleistocene and also of the Holocene (Grossheim 1936, Kharadze 1960.Autochthonous oreo xerophytic species of the Caucasus reveal ancient connections with those of the flora of Asia Minor. Examples are Salvia canescens var. daghestanica, Betonica nivea, species of Silene, Astragalus, Scutellaria, Ziziphora, etc. Members of the autochthonous subnival flora of the Caucasus are also regarded as xerophytic elements of the humid flora of the same region (Kharadze 1960).

The high elevations of the Caucasus display a great variety of life forms, which can be explained for by their evolution under different climatic conditions, strong relief, and habitat fragmentation, and also by the situation of the Caucasus at the crossway of contrasting physical and biogeographical regions (Nakhutsrishvili et al. 2017). The resultant postglacial vegetation has its roots in the Oligocene during which all high-elevation vegetation belts were represented. The first treeless formations, grass and shrubs appeared in the Upper Miocene. The vegetation has changed dramatically during glacial periods. Warm microhabitats in sheltered locations have contributed to the survival even during very cold periods. The postglacial xerophilization in the Caucasus resulted in a degradation of the meso thermophilous forest vegetation (Shatilova et al. 2011). The oroxerophilous vegetation of Irano-Turanian origin radiated into the Central Caucasus through narrow rocky gorges. This vegetation includes cushion-like tragacanths such as Astragalus denudatus and forbs like Artemisia splendens, A. sosnovskyi, Silene linearifolia, Astragalus kazbekii, etc. A similar type of vegetation formed under dry continental conditions in the Eastern Caucasus. A number of herbaceous species and communities arrived in the Central Caucasus in the post-glacial period. Of these, the Astragalus bungeanus aggregate, is a characteristic element of the mountain-steppe landscapes with phryganoid vegetation and Scutellaria leptostegia which is typical for the tragacanth vegetation of the subalpine belt of the Tergi gorge (Kharadze 1948a). Other examples of these postglacial elements are Campanula alliariifolia which inhabits talus, often at the edge of birch forests (Grossheim 1948) and particularly Carex buschiorum (a species closely related to $C$. humilis), which became a typical element of postglacial subalpine pine (Pinus kochiana) forests on glacial moraines. Today $P$. kochiana is only found on granite outcrops of the Daryali canyon (the Central Caucasus.As a result of the postglacial warming, birch (Betula litwinowil) forests became strongly reduced, and are now largely confined to park-type woodland and dwarf shrubs ('Krummholzt) in the subalpine belt (Tumadjanov 1947, Gulisashvili 1964, Dolukhanov 2010, Nakhutsrishvili et al. 2017).

Thermophilous meadows with steppe species such as Festuca ovina, F. valesiaca, Bromopsis riparia, Carex buschiorum, Pulsatilla violacea and others became abundant. Kobresia species reached the highlands of the 
Caucasus from the mountains of Central Asia and are found primarily on the wind-swept crests or ridges. The wide distribution of the Festucetum variae type of grassland in the highlands of the Caucasus is also associated with the postglacial xerothermic period (Kimeridze 1965a,b, Nakhutsrishvili 2013, Pysek, Srütek 1989).

\section{Floristic Overview of Vegetation Belts}

In the eastern part of the Central Caucasus, over 1300 species of vascular plants have been recorded (Sakhokia and Khutsishvili 1975, Ketskhoveli et al. 1971-2011.Among them 0.5\% are gymnosperm species, $79.2 \%$ dicots, and $20.3 \%$ monocots (Grossheim 1936). These are: Asteraceae, Lamiaceae, Rosaceae, Caryophylaceae, Boraginaceae, Fabaceae, Liliaceae (in decreasing species richness. The region is especially rich in endemic species (Grossheim 1936, Schatz et al. 2014). The presence of 6 Caucasian endemic genera (out of 11) also underlines the richness of the flora of this region. e.g. Agasyllis, Symphyoloma, Cladochaeta, Dolichorrhiza, Trigonocaryum, and Pseudovesicaria. The highest number of endemic species appears in the genus Campanula (16 species), followed by Alchemilla and Astragalus with at least 10 species each. The genera Thymus, Delphinium, Onobrychis, and Isatis exhibit a 100\% region-specific endemism (Gagnidze 2000, 2005, Nakhutsrishvili 2013, Nakhutsrishvili et al. 2017).

In the vegetation overview we adopt the classical biogeographic zonation of mountain belts by elevation as there are (from low to high): colline, lower montane, mid montane, upper montane, treeline ecotone, alpine (sometimes separated into a lower, mid and upper belt), subnival and nival. However, in addition, the term 'subalpine', often abandoned for its difficult definition (Löve 1970, Körner 2003), will play a prominent role here. For some authors, subalpine is a synonymous to the treeline ecotone, i.e. the belt of gradual opening of the upper montane forest towards the tree limit. For others, this is a synonymous to the upper montane zone. The difficulty in the central Caucasus is that the natural montane forest ecosystems are largely gone and became replaced by vast terrain with many types of different grassland, thus, extending the alpine grassland formation downhill, both by appearance as well, as in some cases, by taxonomy. Hence, traditionally, 'subalpine' has been applied to much of the open land between the potential natural position of the climatic treeline at ca. $2500 \mathrm{~m}$ and sloping down to 1900-2000 m elevation. From an ecological point of view there are good reasons for such a wider application of 'subalpine', given the short stature vegetation shares many of the common features of alpine vegetation, such as a warmer microclimate, strong influence of exposure and microtopography and many common taxa that are found both in the alpine and below alpine grassland. The term upper montane will thus, be restricted to patches of high elevation woodland. In addition, at each elevation, moisture conditions may vary from aquatic, to wet, moist, mesic, dry and very dry, addressed as xerophytic or, because of the mountain position, as oroxerophytic. Notably, that horizontal graduation of life conditions is more pronounced at low elevation, still significant in the subalpine belt, but vanishes at upper alpine and nival elevations, where moisture conditions become more similar across the Caucasus range (Nakhutsrishvili et al. 2017).

\section{Materials and Methods}

\section{Ethnobotanical studies}

From 2013 to 2019 we interviewed over 380 participants in all regions of Georgia not occupied by Russian forces on their general plant use, recording over 32000 individual uses. The analyses of all uses have been published in a variety of papers (Batsatsashvikli et al. 2020a,b; Bussmann 2017; Bussmann et al. 2016a,b,c, 2017a,b,c, 2018, 2020a,b,c,d,e,f,g,h,i,j,k; 2021a,b,c). However, of all uses over 19800 mentions were of food plants, which is why we regarded it as prudent to present a separate analysis of these. Interviews using semi-structured questionnaires were conducted after obtaining the oral prior informed consent of the participants (Mir et al., 2021; Hassan et al., 2021), which were selected by snowball sampling, trying to reach gender balance, and representing different age-groups. Most participants were however over 50 years old, as interviews targeted remote villages where only very few younger people remain. All interviews were carried out in the participants' homes and gardens by native speakers of Georgian and its dialects (Imeretian, Rachian, Lechkhumian, Tush, Khevsurian, Psavian, Kakhetian), other Kartvelian languages (Megrelian, Svan) and minority languages (Ossetian, Ude, Azeri, Armenian, Greek). Plants grown in home gardens were used as prompts, while wild-collected species were free listed. We classified species as "garden" when they were grown/collected in cultivated areas, and as "forest / wild-collected" when growing and harvested in the wild. We maintained the distinction of "forest" and "garden" because it was used in our previous publications from the region (Batsatsashvikli et al. 2020a,b; Bussmann 2017; Bussmann et al. 2016a,b,c, 2017a,b,c, 2018, 2020a,b,c,d,e,f,g,h,i,j,k; 2021a,b,c), to maintain consistency. In contrast to many other countries Georgia benefits from a complete flora (Flora of Georgia Committee 1941-1952, 1971-2011; Gagnidze 2005; Makashvili 1952-1953, 1991) and a broad inventory of vernacular names in all languages (Makashvili 1991). Species were 
identified directly in the field, using this literature, and vouchers collected and deposited in the National Herbarium of Georgia (TBI). The nomenclature of all species follows www.tropicos.org, under APGIII (Angiosperm Phylogeny Group 2009). Collection permits were provided through the Institute of Botany, Ilia State University, Tbilisi.

\section{Vegetation Sampling and plant identification}

A total of 619 plots with sizes from $5 \mathrm{~m}^{2}$ (rock vegetation) to $25 \mathrm{~m}^{2}$ (alpine herb communities) and $100 \mathrm{~m}^{2}$ (alpine pastures) were selected. For each sampling site, altitude, slope, and exposition were noted, and relative values of density, frequency, cover and Importance Value (IV) were calculated following the methodology of Curtis and McIntosh (1950), Son et al. 2019), Tian et al. 2018). Geographic coordinates were recorded for each sampling site. The expositions of the mountains i.e., east (E), west $(\mathrm{W})$, south $(\mathrm{S})$ and north $(\mathrm{N})$ were determined with the help of clinometer, latitude, longitude, and altitude using geographical positioning system (GPS). Plant specimens were collected, and standard herbarium vouchers prepared (ljaz et al., 2018). All the specimens were identified with the help of Flora of Georgia (Flora of Georgia Committee, 1941-52, 1972-2011, Gagnize 2005) and other available literature citations required. Scientific names of plant species were cross-checked and updated through www.theplantlist.org and www.tropicos.org. The plant specimens were deposited in the National Herbarium of Georgia.

\section{Statistical analyses}

All the collected data of plant species and other environmental variables data were used to find out the relationship among them (Mayor et al., 2017, Smilauer and Jan, 2014). Matrixes of IV data of all the recorded plant species (563 species) from 619 plots were used in the analyzes. The analyses were carried using PCORD 5.0 and RStudio 4.0.0 (R Core Team, 2020) at $5 \%$ of probability.

\section{Species area curves (SAC) and Two-way indicator species analysis (TWINSPAN)}

Species area curves $(S A C)$ were produced using PC-ORD to check the efficiency of the sampling effort, where plant abundance data with Sorensen distance values were used to create species area curves. For the classification of the recorded plant species (563 species) and 619 plots, Two-way indicator species analysis (TWINSPAN) were processed using PC-ORD version 5.0 to identify the major plant communities (Haq et al., 2017, Hill, 1979, Rahman et al., 2020). These analyses were based on patterns of similarity via Sorenson Distance Measurements using Wards Linkage Method (Greig-Smith, 1983) and IV (Rahman et al., 2020). Similarly, the plant communities were named on the basis of dominant species (Lou et al., 2016, Son et al., 2019, Song et al., 2015, Tian et al., 2018).

\section{Community association and species composition}

We visualized floristic relation among communities and environmental variables through a Principal Component Analysis (PCA) (Terzi et al., 2019) using the package "vegan" (Oksanen et al., 2019). To observe which environmental variable (altitude and different aspects) significantly influence communities' distribution in the ordination, we used the "envfit" function with 999 permutations using the package "vegan". The vectors projected on points had a maximum correlation with corresponding environmental variables.

To evaluate a potential variation in species composition between plant communities, we applied a Permutational Multivariate Analysis of Variance (PERMANOVA) with Euclidean distance and 999 permutations. Pairwise comparisons between communities with corrections for multiple testing were conducted using Euclidean distance and 999 permutations. False discovery rate (FDR) was used as p-value adjustment method. PERMANOVA and pairwise comparisons were conducted with the "RVAideMemoire" package (Hervé, 2020).

\section{Partial CCA}

To observe how explanatory variables drive the plant communities, we conducted a canonical correspondence analysis (CCA) and variation partitioning tests (partial CCA). We fit the best model using the variables that most explain the variance by conducting the step function in the "stats" package. We also checked multicollinearity between variables using the Variance Inflation Factor (VIF). Then, we carried out CCA and partial CCA using the package "vegan".

\section{Regression models}

To compare if there is variation in the average altitude among plant communities, we used a Generalized Linear Model (GLM) followed by Likelihood Ratio test using the "stats" package (R Core Team, 2020.The altitude was fit as 
response variable and the communities as explanatory variables. Pairwise comparisons were conducted with estimated marginal means using the package "emmeans" (Lenth, 2020). To compare the species richness, Shannon diversity, Simpson diversity and Pielou's evenness between plant communities, we conducted a GLM with Gaussian error distribution (except for species richness, in which we used negative binomial distribution controlling for overdispersion) followed by Likelihood Ratio test using the 'stats' and 'car' (Fox and Weisberg, 2018) packages, respectively.

\section{Diversity index}

To evaluate the beta diversity patterns between the seven communities, the Sørensen dissimilarity index was decomposed in two additive components: the spatial turnover (Simpson pairwise dissimilarity) and nestednessresultant components (nestedness-fraction of Sorensen pairwise dissimilarity) (Baselga, 2010, 2013, Baselga and Orme, 2012). These analyses were conducted using the "betapart" package (Baselga et al., 2018).

\section{Results and Discussion}

\section{Use of the Alpine and Subalpine flora}

We found that 183 species of the subalpine and alpine flora, representing about 33\% of all species encountered in the vegetation survey, had reported uses. Eighty-four species were used as animal fodder, especially in summer pastures, 66 species were used medicinally, 65 species were used as food, especially to make phkhali (herb pie), khachapuri (herb and cheese filled bread), in sat'sebai (dipped in yogurt and milk), or lacto-fermented, 18 species as utensils, i.e., to make brooms, four for cultural purposes (e.g., as ornamentals and toys), two for construction purposes, and one species was used as fuelwood. A complete overview on all species and their uses is given in Table 1.

\section{Plant use history}

The Caucasus is regarded as global biodiversity hotspot (Akhalkatsi \& Tarkhnishvili 2012; Otte et al. 2011; Schatz et al. 2009; Kimeridze \& Akhalkatsi 2006), and Georgia has its fair share of the tremendous diversity of the region (Schatz et al. 2009.Botanical exploration of the Caucasus has a long history, yielding good recent treatments of the area's vegetation, in particular with regard to Georgia (Nakhutsrishvili 1999). As such Georgia has long been the center point of botanical Exploration in the Caucasus, with Bakuriani alpine Botanical Garden serving as a hub. The visitor's log of the garden reads like a "Who is who" of $20^{\text {th }}$ century Botany.

The territory of modern-day Georgia has been continuously inhabited since the early Stone Age, and agriculture was developed during the early Neolithic era (Javakhishvili 1987). In Georgian the name of the country is "Sakartvelo", and "Georgia" is semantically linked to Greek ( $ү \varepsilon \omega p ү i \alpha)$ meaning "agriculture" (Javakhishvili 1987). Human occupation however started in the Early Pleistocene. The 1.7-Myr-old hominid fossils of Dmanisi in Southern Georgia are the earliest known hominid-site outside of Africa (Finlayson 2005, Gabunia, Vekua 1995, Gabunia et al. 2000). This specimen has been classified as In the Late Middle Paleolithic and Early Upper Neanderthal and modern human occupation are well documented (Adler, Bar-Oz 2009). Upper Paleolithic fossils of Dzudzuana Cave include remnants of wool (Capra caucasica) and dyed fibers of wild flax (Linum usitatissimum L.) dated to 36-34 Ka BP (Adler, Bar-Oz 2009).

The archeological findings from Neolithic and Early Bronze periods are rich with plant fossils and seeds of both wild species and local landraces. Seven species of cultivated wheat - Triticum aestivum L., T. carthlicum Nevski, T. compactum Host, T. dicoccum Schrank, T. macha Dekapr., Menabde, T. monococcum L., T. spelta L., one wild relative, Aegilops cylindrica Host., as well as millet - Panicum milliaceum L., barley - Hordeum vulgare L., Italian millet Setaria italica L.) P. Beauv., oats - Avena sativa L., wild lentil - Lens ervoides (Brignolidi, Brunhoff) Grande, and pea Pisum sativum L. have been discovered in Arukhlo, dating back to the 6th - 2nd millennium BC (Melikishvili 1970). The earliest grapevine seeds indicating cultivation were excavated in southern Georgia and date to $~ 8.000$ years BP (Ramishvili 1988). Due to its long tradition, agriculture in Georgia is characterized by a great diversity of landraces, and endemic species of crops. These show a high level of adaptation to local climatic conditions and often-high disease resistance. Early research documented this great variety (Dekaprelevich et al. 1929, Ketskhoveli 1928, 1957, Ketskovili et al. 1960, Menabde 1938, 1948), but a rapid loss of local cultivars of cereals, legumes and flax began in the 1950s with Stalinist agricultural reform (Akhaljatsi 2009, Akhalkatsi et al., 2010). Despite the long cultural history, recent studies on cultivated plants are rather scarce (e.g., Pistrick et al. 2009, Zhizhizlashvili et al. 1980). 
Table 1. Uses of Alpine plant species in Georgia

\begin{tabular}{|c|c|c|c|}
\hline Family & Species & $\begin{array}{l}\text { Local Name (Georgian, if not } \\
\text { indicated otherwise in parenthesis: } \\
\text { Arm. = Armenian; Imer. = Imeretian; } \\
\text { Khev. = Khevsurian); Psha. = Pshaviab; } \\
\text { Rach. = Rachian; Russ. = Russian; Svan. } \\
\text { = Svanetian; Tush. = Tushetian) }\end{array}$ & Use Category \\
\hline Amaryllidaceae & Allium kunthianum Vved. & 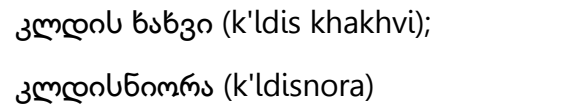 & Food - bulbs eaten raw \\
\hline \multirow{11}{*}{ Apiaceae } & Agasyllis latifolia (M. Bieb.) Boiss. & 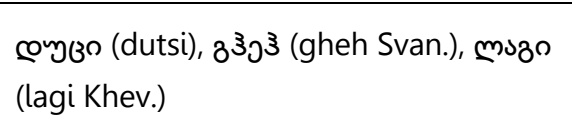 & $\begin{array}{l}\text { Food - leaves, petiole, stem for khachapuri, phkhali, pickled } \\
\text { Medicinal - leaves, stem, petioles as anthelmintic, stem bark for } \\
\text { asthma, root for the digstive system }\end{array}$ \\
\hline & $\begin{array}{l}\text { Anthriscus nemorosa (M. Bieb.) } \\
\text { Spreng. }\end{array}$ & 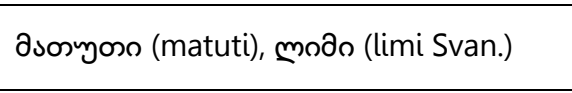 & $\begin{array}{l}\text { Food - fruits eaten as spice, leaves and stems pickled } \\
\text { Plant is regarded as toxic }\end{array}$ \\
\hline & Astrantia maxima Pall. & Уззсоззs (uk'vdava) & Cultural - whole plant as decoration \\
\hline & Astrantia trifida Hoffm. & & Cultural - whole plant as decoration \\
\hline & Astrodaucus orientalis Drude & 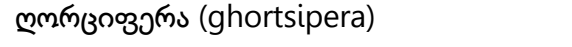 & \\
\hline & Bupleurum polyphyllum Ledeb. & & \\
\hline & Carum caucasicum Boiss. & & Animal food - leaves / whole plant grazed and as fodder \\
\hline & Chaerophyllum aureum $\mathrm{L}$. & 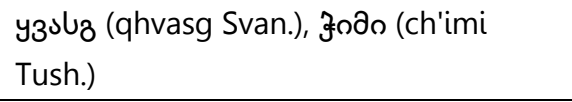 & $\begin{array}{l}\text { Food - root eaten raw, stems pickled } \\
\text { Medicinal - root for nerves }\end{array}$ \\
\hline & Chaerophyllum humile M. Bieb. & & Animal food - leaves / whole plant grazed and as fodder \\
\hline & Chaerophyllum roseum M. Bieb. & & Animal food - leaves / whole plant grazed and as fodder \\
\hline & Chaerophyllum rubellum Albov. & & Animal food - leaves / whole plant grazed and as fodder \\
\hline
\end{tabular}


Ethnobotany Research and Applications

\begin{tabular}{|c|c|c|c|}
\hline & $\begin{array}{l}\text { Chamaesciadium acaule (M. Bieb.) C.A. } \\
\text { Mey. }\end{array}$ & 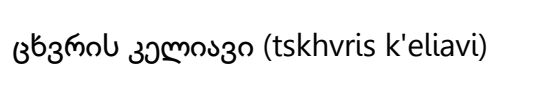 & \\
\hline & Heracleum asperum M. Bieb. & פy3ysn (shupq'a) & $\begin{array}{l}\text { Food - leaves and stems pickled, for sats'ebai and eaten raw } \\
\text { Medicinal - root for toothache }\end{array}$ \\
\hline & Heracleum sosnowskyi M. Bieb. & $\begin{array}{l}\text { عoyn (diq'i); Jjto (qekhi Arm.); hoh3s } \\
\text { (chichva Svan.) }\end{array}$ & $\begin{array}{l}\text { Food - leaves and stems pickled, for sats'ebai, chave and eaten } \\
\text { raw } \\
\text { Medicinal - root to treat cancer and tumors }\end{array}$ \\
\hline & Ligusticum alatum Spreng. & asmosas (mariama) & Food - leaves eaten raw and in sats'ebai \\
\hline & Pastinaca armena Fisch. \& C.A. Mey. & & Animal food - leaves / whole plant grazed and as fodder \\
\hline & Pimpinella rhodantha Boiss. & & Animal food - leaves / whole plant grazed and as fodder \\
\hline & Pimpinella saxifraga $\mathrm{L}$. & 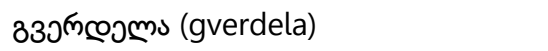 & Animal food - leaves / whole plant grazed and as fodder \\
\hline & Seseli alpinum M. Bieb. & & Animal food - leaves / whole plant grazed and as fodder \\
\hline & $\begin{array}{l}\text { Seseli transcaucasicum Pimenov \& } \\
\text { Sdobnina }\end{array}$ & 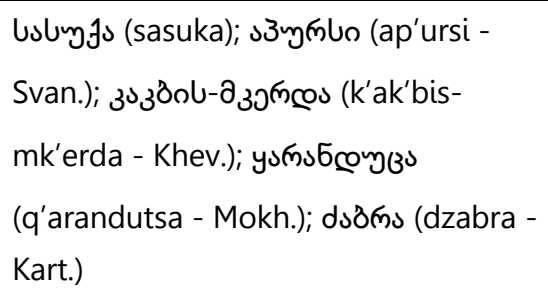 & Animal food - leaves / whole plant grazed and as fodder \\
\hline & Symphyoloma graveolens C.A. Mey. & & \\
\hline Aspleniaceae & Asplenium septentrionale (L.) Hoffm. & & \\
\hline \multirow{5}{*}{ Asteraceae } & Achillea millefolium $\mathrm{L}$. & 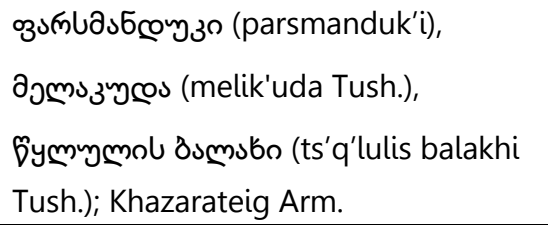 & $\begin{array}{l}\text { Food - leaves in khachapuri, whole plant as tea } \\
\text { Medicinal - Leaves as anti-inflammatory, cholagogue, diuretic, } \\
\text { inflammation, kidneys, liver, sore throat, ulcers, wounds, whole } \\
\text { plant as panacea } \\
\text { Utensils and Tools - leaves as dye }\end{array}$ \\
\hline & $\begin{array}{l}\text { Achillea ptarmicifolia (Willd.) Rupr. ex } \\
\text { Heimerl }\end{array}$ & 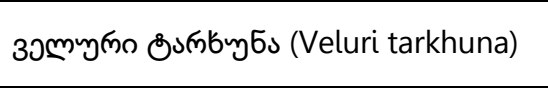 & Medicinal - leaves for wounds \\
\hline & Aetheopappus caucasicus Sosn. & 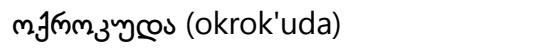 & \\
\hline & Antennaria caucasica Boriss. & & \\
\hline & Anthemis iberica M. Bieb. & & \\
\hline
\end{tabular}


Ethnobotany Research and Applications

\begin{tabular}{|c|c|c|}
\hline $\begin{array}{l}\text { Anthemis macroglossa Sommier \& } \\
\text { Levier }\end{array}$ & & \\
\hline Anthemis marschalliana Willd. & & \\
\hline Anthemis rudolphiana M. Bieb. & & \\
\hline Anthemis sosnovskyana Fed. & & \\
\hline Arctium lappa $\mathrm{L}$. & domb $30^{5}$ s (dzirxvena); Graduk (Arm.) & $\begin{array}{l}\text { Food - leaves and roots eaten raw, leaves in phkhali, stems } \\
\text { pickled } \\
\text { Medicinal - flowers and leaves as antibiotic }\end{array}$ \\
\hline Artemisia absinthium $\mathrm{L}$. & 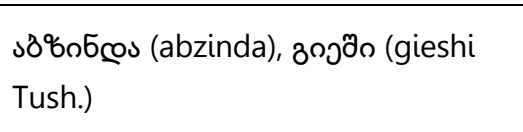 & $\begin{array}{l}\text { Food - leaves eaten raw and as tea } \\
\text { Medicinal - leaves to treat bedwetting in children, cold, sore } \\
\text { throat, fever }\end{array}$ \\
\hline Artemisia campestris L. & & Utensils and tools - stem for brooms \\
\hline Artemisia chamaemelifolia Vill. & & Utensils and tools - stem for brooms \\
\hline Artemisia marschalliana Spreng. & ふ๐ృð̊ (gieshi) & Utensils and tools - stem for brooms \\
\hline Artemisia splendens Willd. & & \\
\hline Artemisia vulgaris $\mathrm{L}$. & 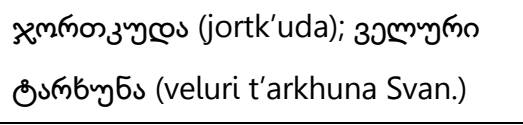 & $\begin{array}{l}\text { Food - leaves eaten raw and in sats'ebai } \\
\text { Medicinal - leaves as diuretic, for gallbladder and to kill lice }\end{array}$ \\
\hline Aster alpinus L. & & \\
\hline Aster ibericus Steven ex M. Bieb. & & \\
\hline $\begin{array}{l}\text { Carduus onopordioides Fisch. ex M. } \\
\text { Bieb. }\end{array}$ & 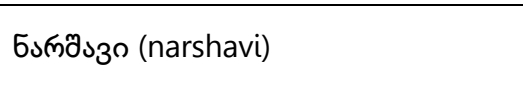 & \\
\hline Centaurea cheiranthifolia Willd. & & \\
\hline Centaurea salicifolia M. Bieb. & & \\
\hline Cicerbita racemosa (Willd.) Beauverd & & \\
\hline Cirsium echinus Hand.-Mazz. & Esmo (nari) & $\begin{array}{l}\text { Food - leaves for sats'ebai } \\
\text { Medicinal - leaves for hemorrhoids }\end{array}$ \\
\hline Cirsium obvallatum M. Bieb. & Esmo (nari) & $\begin{array}{l}\text { Food - leaves for sats'ebai } \\
\text { Medicinal - leaves for hemorrhoids }\end{array}$ \\
\hline Cirsium osseticum Petr. & Esmo (nari) & $\begin{array}{l}\text { Food - leaves for sats'ebai } \\
\text { Medicinal - leaves for hemorrhoids }\end{array}$ \\
\hline Cirsium pugnax Sommier \& Levier & Esmo (nari) & $\begin{array}{l}\text { Food - leaves for sats'ebai } \\
\text { Medicinal - leaves for hemorrhoids }\end{array}$ \\
\hline
\end{tabular}


Ethnobotany Research and Applications

\begin{tabular}{|c|c|c|}
\hline Cirsium rhizocephalum C.A. Mey. & Esmo (nari) & $\begin{array}{l}\text { Food - leaves for sats'ebai } \\
\text { Medicinal - leaves for hemorrhoids }\end{array}$ \\
\hline Cirsium simplex C.A. Mey. & Esmo (nari) & $\begin{array}{l}\text { Food - leaves for sats'ebai } \\
\text { Medicinal - leaves for hemorrhoids }\end{array}$ \\
\hline Crepis sonchifolia (M. Bieb.) C.A. Mey. & 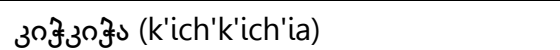 & \\
\hline \multicolumn{3}{|l|}{ Dolichorrhiza renifolia Galushko } \\
\hline \multicolumn{3}{|l|}{$\begin{array}{l}\text { Doronicum macrophyllum Fisch. ex } \\
\text { Hornem. }\end{array}$} \\
\hline \multicolumn{3}{|l|}{ Erigeron alpinus L. } \\
\hline \multicolumn{3}{|l|}{ Erigeron caucasicus Steven } \\
\hline \multicolumn{3}{|l|}{ Erigeron orientalis Boiss. } \\
\hline \multirow{2}{*}{\multicolumn{3}{|c|}{$\begin{array}{l}\text { Erigeron sp. } \\
\text { Erigeron uniflorus } \mathrm{L} .\end{array}$}} \\
\hline & & \\
\hline Gnaphalium supinum L. & ১jஙூymo (dzeruli) & \\
\hline Hieracium elisabethae M. Bieb. & bs๓nбyłs (kharnuka) & \\
\hline Hieracium laevigatum Willd. & bsজmbyłs (kharnuka) & \\
\hline Hieracium panaeoliforme Üksip & 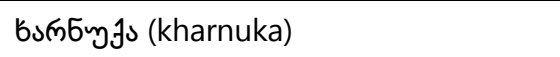 & \\
\hline Hieracium pilosella L. & 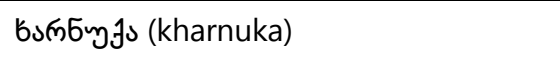 & \\
\hline Hieracium umbellatum $\mathrm{L}$. & bs๓nбyłs (kharnuka) & \\
\hline \multicolumn{3}{|l|}{ Inula orientalis Lam. } \\
\hline \multicolumn{3}{|l|}{ Jurinea filicifolia Boiss. } \\
\hline \multicolumn{3}{|l|}{ Jurinella subacaulis Iljin } \\
\hline Lapsana grandiflora M. Bieb. & 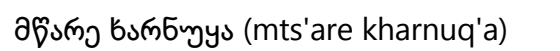 & Food - leaves eaten raw and in soup \\
\hline \multicolumn{3}{|l|}{ Lapsana intermedia M. Bieb. } \\
\hline Leontodon caucasicus Fisch. & 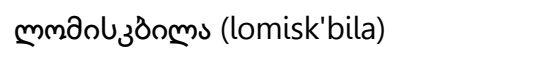 & \\
\hline Leontodon danubialis Jacq. & mmå৮/ठ̇nms (lomisk'bila) & \\
\hline Leontodon hispidus L. & 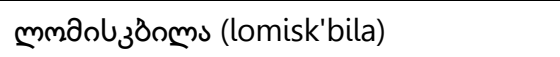 & \\
\hline Leucanthemum vulgare Lam. & 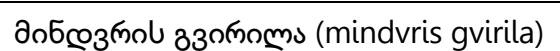 & \\
\hline
\end{tabular}


Ethnobotany Research and Applications

\begin{tabular}{|c|c|c|}
\hline \multicolumn{3}{|l|}{ Ligularia sibirica (L.) Cass. } \\
\hline Matricaria chamomilla L. & $\begin{array}{l}\text { з3опоммs (gvirila); Ромашка } \\
\text { (Romaschka Russ.) }\end{array}$ & $\begin{array}{l}\text { Food - leaves and whole plant for chave, tea } \\
\text { Medicinal - whole plant for cough, diuretic, gastro-intestinal } \\
\text { system, stomach, wounds; leaves for toothache }\end{array}$ \\
\hline Petasites fominï Bordz. & ১yoms (buera) & \\
\hline \multicolumn{3}{|l|}{$\begin{array}{l}\text { Podospermum armeniaca Boiss. \& A. } \\
\text { Huet }\end{array}$} \\
\hline \multicolumn{3}{|l|}{ Psephellus dealbatus (Willd.) K. Koch. } \\
\hline \multicolumn{3}{|l|}{ Pyrethrum coccineum (Willd.) Vorosch. } \\
\hline \multicolumn{3}{|l|}{ Pyrethrum demetrii Manden. } \\
\hline \multicolumn{3}{|l|}{ Pyrethrum fruticulosum Biehl. } \\
\hline Pyrethrum parthenifolium Willd. & з3оmомฺs (gvirila) & $\begin{array}{l}\text { Medicinal - leaves for inflammation, flu, oral inflammation, } \\
\text { toothache }\end{array}$ \\
\hline Pyrethrum roseum (Adams) M. Bieb. & usmfy̆ms (sarts'q'ila) & Medicinal - leaves for wounds \\
\hline Scorzonera biebersteinii Lipsch. & 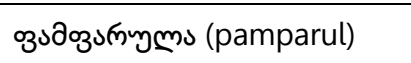 & \\
\hline Scorzonera filifolia Boiss. & оз১дозып̆yms (pamparul) & \\
\hline $\begin{array}{l}\text { Senecio aurantiacus (Hoppe ex Willd.) } \\
\text { Less. }\end{array}$ & 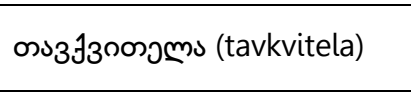 & \\
\hline Senecio karjaginï Sofieva & 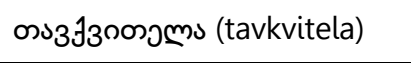 & \\
\hline Senecio rhombifolius (Willd.) Sch. Bip. & 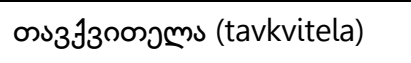 & \\
\hline Senecio taraxacifolius (M. Bieb.) DC. & 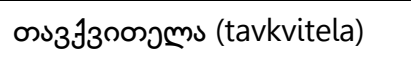 & \\
\hline \multicolumn{3}{|l|}{ Solidago sosnovskyi M. Bieb. } \\
\hline Solidago virgaurea $\mathrm{L}$. & 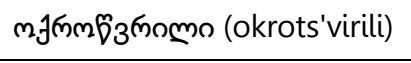 & \\
\hline Tanacetum vulgare $\mathrm{L}$. & 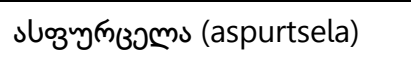 & \\
\hline Taraxacum campylodes G.E. Haglund & 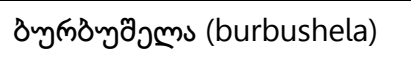 & Animal food - leaves / whole plant grazed and as fodder \\
\hline
\end{tabular}




\section{Ethnobotany Research and Applications}

\begin{tabular}{|c|c|c|c|}
\hline & Taraxacum confusum Schichk. & 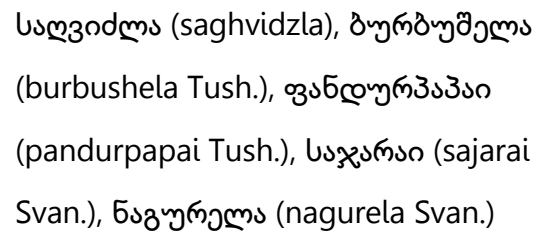 & $\begin{array}{l}\text { Food - leaves for chave, phkhali, tea, eaten raw } \\
\text { Cultural - stems as whistles } \\
\text { Medicinal - leaves for gallstones, diuretic, laxative, toothache, oral } \\
\text { inflammation }\end{array}$ \\
\hline & Taraxacum porphyranthum Boiss. & 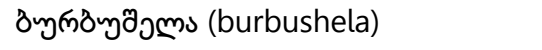 & Animal food - leaves / whole plant grazed and as fodder \\
\hline & Taraxacum stevenï DC. & 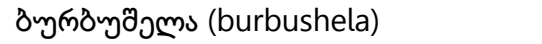 & Animal food - leaves / whole plant grazed and as fodder \\
\hline & Tephroseris karjaginii Holub & & \\
\hline & Tragopogon filifolius Rehm. ex Boiss. & 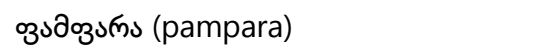 & Food - leaves and root eaten raw, stems pickled \\
\hline & Tragopogon graminifolius DC. & 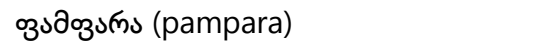 & Food - leaves and root eaten raw, stems pickled \\
\hline & $\begin{array}{l}\text { Tragopogon reticulatus Boiss. \& A. } \\
\text { Huet }\end{array}$ & 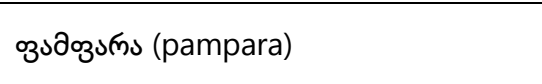 & Food - leaves and root eaten raw, stems pickled \\
\hline & $\begin{array}{l}\text { Tripleurospermum caucasicum (Willd.) } \\
\text { Hayek }\end{array}$ & & \\
\hline & Tripleurospermum subnivale Pobed. & & \\
\hline Athyriaceae & Athyrium filix-femina (L.) Roth & & \\
\hline Berberidaceae & Berberis vulgaris $\mathrm{L}$. & 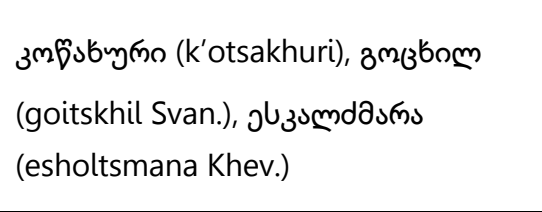 & $\begin{array}{l}\text { Food - fruit eaten raw, as condiment, in tqhemali, as tea, leaves as } \\
\text { tea } \\
\text { Medicinal - fruit for gallblader and liver, leaves for hyperytension } \\
\text { Utensils and tools - leaves and roots as dye } \\
\text { Construction - stems as posts }\end{array}$ \\
\hline Betulaceae & Betula litwinowii Doluch. & 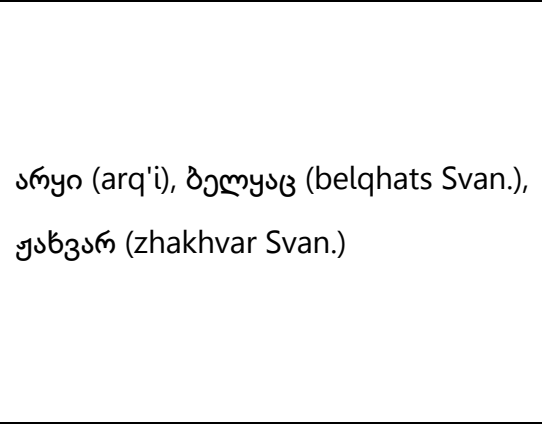 & $\begin{array}{l}\text { Construction - stems as timber } \\
\text { Food - juice drunk raw } \\
\text { Fuel - bark and stems as Firestarter and firewood } \\
\text { Medicinal - Juice for arthritis and goiter, leaves for cold, dandruff, } \\
\text { as panacea, bark for dandruff, hair loss, fruits applied to wounds, } \\
\text { toothache } \\
\text { Utensils and tools - branches as brooms, stems for beer ladles, } \\
\text { bowls, candlesticks, carts, cups, dippers, furniture, plows, sleds, } \\
\text { spinning wheels, spoons, tool handles, vessels for alcohol, yokes } \\
\text { and walking sticks }\end{array}$ \\
\hline
\end{tabular}


Ethnobotany Research and Applications

\begin{tabular}{|c|c|c|c|}
\hline \multirow{14}{*}{ Boraginaceae } & Aipyanthus echioides Steven & & \\
\hline & Aipyanthus pulchra Kolak. & & \\
\hline & Cynoglossum officinale $\mathrm{L}$. & dsmmon jбs (dzaghlis ena) & \\
\hline & Cynoglossum viride Eastw. & & \\
\hline & Echium maculatum L. & 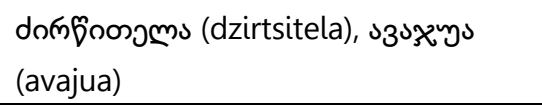 & \\
\hline & Echium rubrum Forssk. & 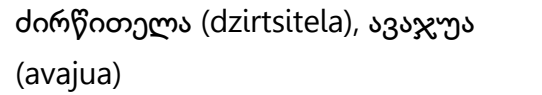 & \\
\hline & Eritrichium caucasicum Grossh. & & \\
\hline & Lappula barbata (M. Bieb.) Gürke & & \\
\hline & Myosotis alpestris F.W. Schmidt & 3อไง6ว (k'esane) & \\
\hline & Myosotis arvensis (L.) Hill. & 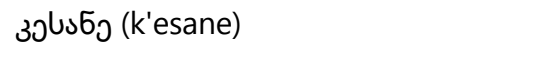 & \\
\hline & Myosotis sylvatica Ehrh. ex Hoffm. & 3อไsб ว (k'esane) & \\
\hline & Nonea rosea (M. Bieb.) Link & & \\
\hline & Symphytum asperum Lepechin & 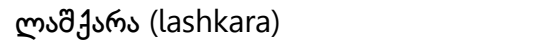 & \\
\hline & Trigonocaryum involucratum Kusn. & & \\
\hline \multirow{8}{*}{ Brassicaceae } & Alyssum alyssoides (L.) L. & & \\
\hline & Arabidopsis thaliana (L.) Heynh. & & \\
\hline & Bunias orientalis L. & 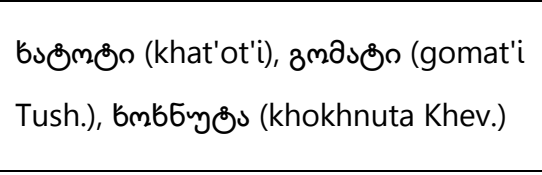 & $\begin{array}{l}\text { Food - leaves for chave and eaten raw, pickled, phkhali, stems } \\
\text { pickled, flowers eaten raw } \\
\text { Medicinal - leaves against poisoning, hangover, snakebite, seeds } \\
\text { as anthelmintic and against parasites }\end{array}$ \\
\hline & Capsella bursa-pastoris (L.) Medik. & 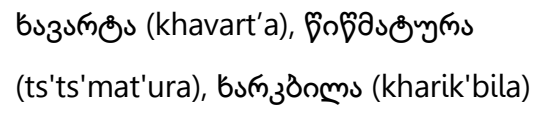 & Food - leaves eaten raw and in phkhali \\
\hline & Dentaria microphylla Willd. & & \\
\hline & Draba bryoides DC. & łycoybs (ch'uduna) & \\
\hline & Draba siliquosa M. Bieb. & łycoy6s (ch'uduna) & \\
\hline & Draba supranivalis Rupr. & łycoy5s (ch'uduna) & \\
\hline
\end{tabular}




\section{Ethnobotany Research and Applications}

\begin{tabular}{|c|c|c|c|}
\hline & Erysimum substrigosum N. Busch & & \\
\hline & Eunomia rotundifolia C.A. Mey. & & \\
\hline & $\begin{array}{l}\text { Pseudo vesicaria digitata (C.A. Mey.) } \\
\text { Rupr. }\end{array}$ & & \\
\hline & Sisymbrium erucastrifolium Trautv. & змбазmms (gongola) & \\
\hline & Sisymbrium loeselii $\mathrm{L}$. & змбамms (gongola) & \\
\hline & Thlaspi pumilum Ledeb. & & \\
\hline \multirow{16}{*}{ Campanulaceae } & $\begin{array}{l}\text { Asyneuma campanuloides (M. Bieb. ex } \\
\text { Sims) Bornm. }\end{array}$ & & \\
\hline & Campanula annae Kolak. & & \\
\hline & Campanula bellidifolia Adams & & Food - flowers eaten raw \\
\hline & Campanula biebersteiniana C.A. Mey. & 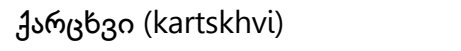 & Food - flowers eaten raw \\
\hline & Campanula ciliata Thunb. & & \\
\hline & Campanula collina M. Bieb. & & \\
\hline & $\begin{array}{l}\text { Campanula hohenackeri Fisch. \& C.A. } \\
\text { Mey. }\end{array}$ & & \\
\hline & Campanula hypopolia Trautv. & & \\
\hline & Campanula latifolia L. & ১লmmə̃s (alosha) & \\
\hline & $\begin{array}{l}\text { Campanula oblongifolia (K. Koch) } \\
\text { Kharadze }\end{array}$ & & \\
\hline & Campanula petrophila Rupr. & & \\
\hline & Campanula rapunculoides $\mathrm{L}$. & 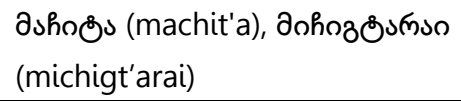 & Food - flower eaten raw, leaves for sats'ebai \\
\hline & Campanula saxifraga M. Bieb. & & \\
\hline & Campanula sosnowskyi Kharadze & & \\
\hline & Campanula trautvetteri Grossh. ex Fed. & & \\
\hline & Campanula tridentata Schreb. & & \\
\hline \multirow{2}{*}{ Caprifoliacae } & Valeriana alpestris Steven & 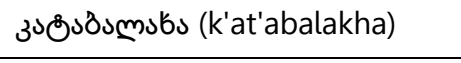 & \\
\hline & Valeriana cardamines M. Bieb. & 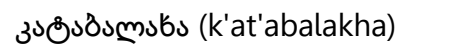 & \\
\hline
\end{tabular}




\section{Ethnobotany Research and Applications}

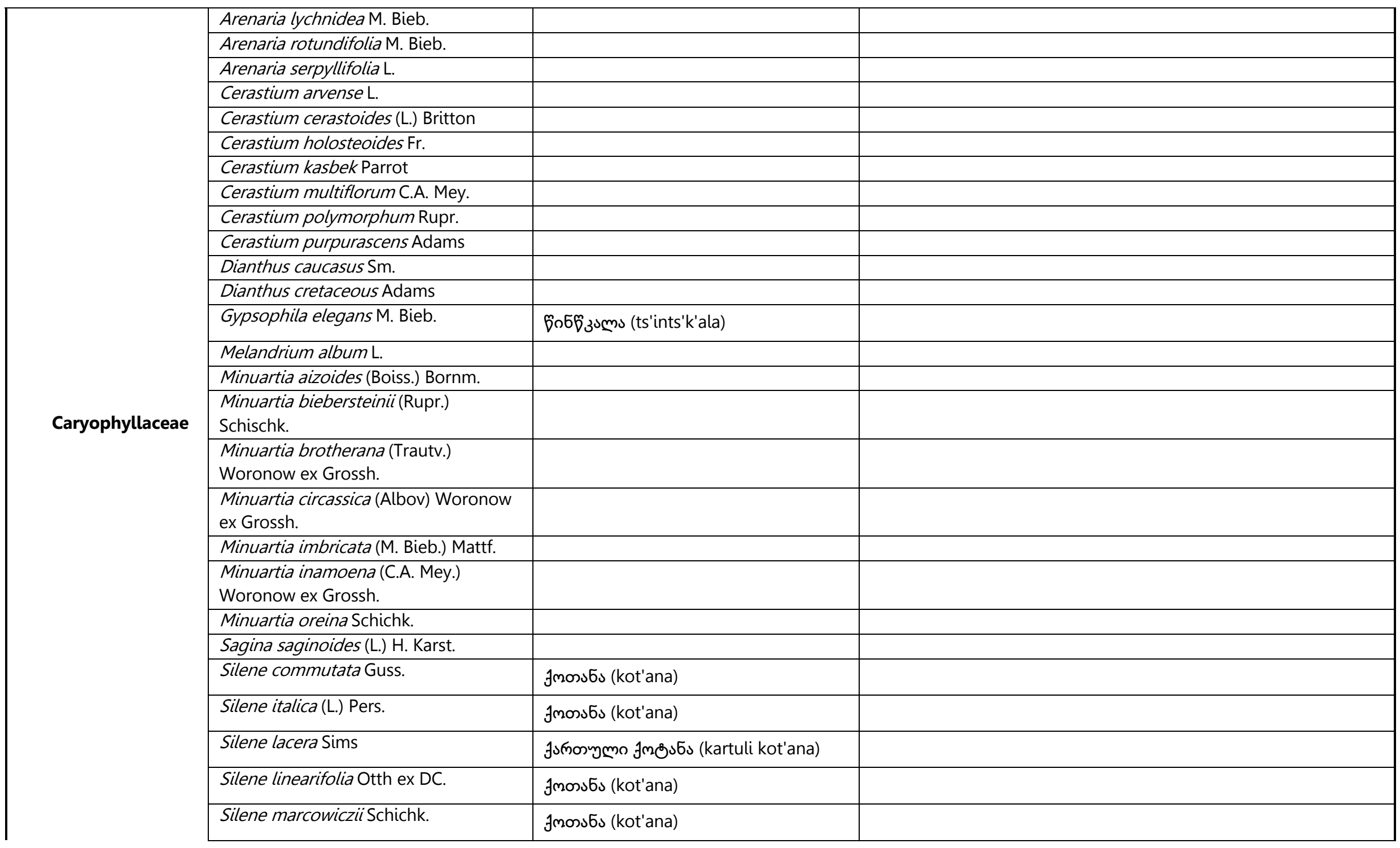


Ethnobotany Research and Applications

\begin{tabular}{|c|c|c|c|}
\hline & Silene ruprechtii Schichk. & Jmoss6s (kot'ana) & \\
\hline & Silene wallichiana Klotzsch & ałnз১б১ (mch'ivana) & Food - leaves for phkhali and pickled \\
\hline Chenopodiaceae & Chenopodium botrys $\mathrm{L}$. & & \\
\hline \multirow{4}{*}{ Cistaceae } & Helianthemum grandiflorum DC. & 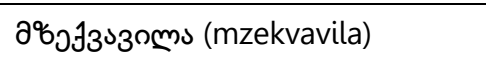 & \\
\hline & Helianthemum nitidum Clementi & а\%っłз১з০লms (mzekvavila) & \\
\hline & Helianthemum nummularium Mill. & а\%っłз১зомু (mzekvavila) & \\
\hline & Helianthemum ovatum Dun. & 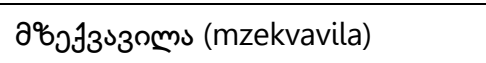 & \\
\hline Colchicaceae & Colchicum umbrosum Steven & ப১ఠিmзмmos (tskhsat'ovlia) & \\
\hline \multirow{7}{*}{ Crassulaceae } & Sedum acre L. & & \\
\hline & Sedum gracile C.A. Mey. & & \\
\hline & Sedum oppositifolium Sims & 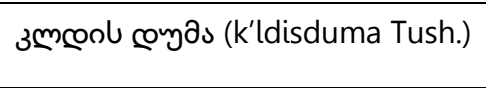 & $\begin{array}{l}\text { Food - leaves eaten raw and in phkhali } \\
\text { Medicinal - leaves for furuncles, toothache, wounds }\end{array}$ \\
\hline & Sedum stoloniferum J.F. Gmel. & auyjs5s (musukana) & \\
\hline & Sedum tenellum M. Bieb. & & \\
\hline & $\begin{array}{l}\text { Sempervivum caucasicum Rupr. ex } \\
\text { Boiss. }\end{array}$ & 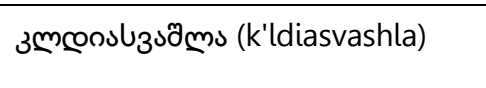 & \\
\hline & Sempervivum pumilum M. Bieb. & 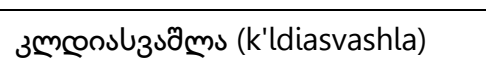 & \\
\hline \multirow[b]{2}{*}{ Cupressaceae } & Juniperus depressa Steven. & łomo (ch'ili) & \\
\hline & $\begin{array}{l}\text { Juniperus hemisphaerica J. Presl \& C. } \\
\text { Presl }\end{array}$ & zmobom (tzqhero Svan.) & Medicinal - branches for kidneys, urinary system, blood cleansing \\
\hline Cuscutaceae & Cuscuta europaea $\mathrm{L}$. & & \\
\hline \multirow{5}{*}{ Cyperaceae } & $\begin{array}{l}\text { Baeothryon pumilum (Vahl) Á. Löve \& } \\
\text { D. Löve }\end{array}$ & & \\
\hline & Blysmus compressus (L.) Panz. ex Link & & \\
\hline & Carex buschiorum V.I. Krecz. & obmo (isli) & \\
\hline & Carex caryophyllea Latourr. & obmo (isli) & \\
\hline & Carex dacica Heuff. & obmo (isli) & \\
\hline
\end{tabular}


Ethnobotany Research and Applications

\begin{tabular}{|c|c|c|c|}
\hline & Carex huetiana Boiss. & obmo (isli) & \\
\hline & Carex medwedewii Leskov & obmo (isli) & \\
\hline & Carex meinshauseniana V.I. Krecz. & obmo (isli) & \\
\hline & Carex microglochin Wahlenb. & obmo (isli) & \\
\hline & Carex pallens Z.P. Wang & obmo (isli) & \\
\hline & Carex pallescens L. & obmo (isli) & \\
\hline & Carex sylvatica Huds. & obmo (isli) & \\
\hline & Carex tristis M. Bieb. & obmo (isli) & \\
\hline & $\begin{array}{l}\text { Kobresia capillifolia (Decne.) C.B. } \\
\text { Clarke }\end{array}$ & & \\
\hline & Kobresia capilliformis Ivanina & & \\
\hline & $\begin{array}{l}\text { Kobresia humilis (C.A. Mey. ex Trautv.) } \\
\text { Serg. }\end{array}$ & & \\
\hline & $\begin{array}{l}\text { Kobresia schoenoides (C.A. Mey.) } \\
\text { Steud. }\end{array}$ & & \\
\hline \multirow{4}{*}{ Dipsacaceae } & Cephalaria gigantea (Ledeb.) Bobrov & பзозЈмmм (sk'ip'alo) & $\begin{array}{l}\text { Food - stems pickled } \\
\text { Cultural - stems used by children as arrows } \\
\text { Medicinal - leaves and stems for babies diseases }\end{array}$ \\
\hline & Knautia montana DC. & & \\
\hline & Scabiosa bipinnata C. Koch & & \\
\hline & Scabiosa caucasica M. Bieb. & उ०мпозмпмом (tsilpolio) & \\
\hline Dryopteridaceae & Dryopteris filix-mas (L.) Schott & hocoy5s (chaduna); hoços (chada Tush.) & $\begin{array}{l}\text { Food - leaves pickled, in phkhali and eaten raw } \\
\text { Medicinal - leaves for bruises }\end{array}$ \\
\hline Eleagnaceae & Hippophaë rhamnoides L. & J১330 (katsvi) & Medicinal - fruits for vitamins and diabetes \\
\hline Ephedraceae & Ephedra procera C.A. Mey. & 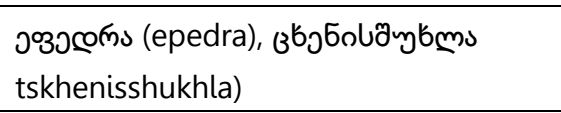 & \\
\hline Equisetaceae & Equisetum arvense $\mathrm{L}$. & $\check{y}_{3 \cap \delta)}$ (shvit'a) & Medicinal - leaves and stems for wounds, kidneys, urinary system \\
\hline
\end{tabular}


Ethnobotany Research and Applications

\begin{tabular}{|c|c|c|c|}
\hline & Equisetum palustre $\mathrm{L}$. & $\eta_{3 \circ \delta}$ (shvit'a) & \\
\hline \multirow{6}{*}{ Ericaceae } & Empetrum caucasicum Juz. & зวด็ว๓ns (k'ets'era) & $\begin{array}{l}\text { Food - fruit eaten } \\
\text { Utensils and tools - branches as brush to wash tools }\end{array}$ \\
\hline & Empetrum nigrum $\mathrm{L}$. & зэڤొכms (k'ets'era) & $\begin{array}{l}\text { Food - fruit eaten } \\
\text { Utensils and tools - branches as brush to wash tools }\end{array}$ \\
\hline & Pyrola rotundifolia $\mathrm{L}$. & & \\
\hline & Rhododendron caucasicum Pall. & 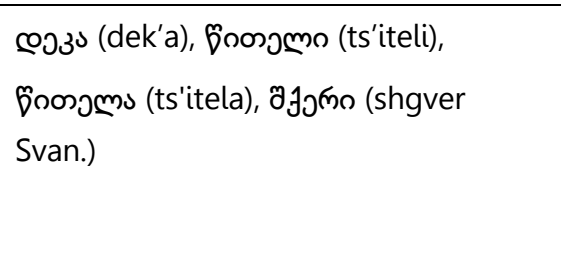 & $\begin{array}{l}\text { Food - branches and leaves to darken beer, flowers eaten raw and } \\
\text { in sats'ebai, leaves for phkhali, leaves, flowers, and fruits for tea } \\
\text { Medicinal - flowers and leaves as anti-inflammatory and for } \\
\text { digestive system, leaves for colds, diuretic, lower sexual potency, } \\
\text { heart } \\
\text { Utensils and tools - leaves as dye }\end{array}$ \\
\hline & Vaccinium myrtillus L. & 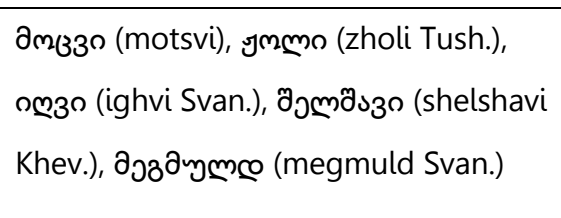 & $\begin{array}{l}\text { Food - fruits eaten raw, for marmalade, tea; leaves, branches, and } \\
\text { flowers for tea } \\
\text { Medicinal - leaves to decrease blood sugar and for kidney stones, } \\
\text { fruits as tea and for dry throat } \\
\text { Utensils and tools - branches for dye }\end{array}$ \\
\hline & Vaccinium vitis-idaea $\mathrm{L}$. & 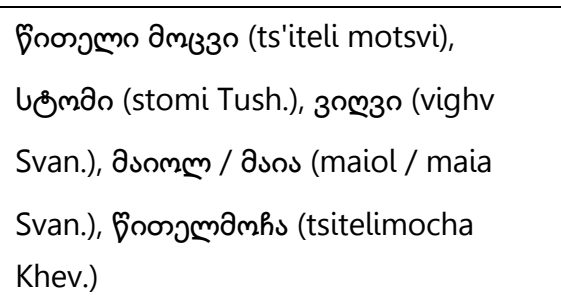 & $\begin{array}{l}\text { Food - fruit eaten raw, branches and leaves for tea } \\
\text { Medicinal - leaves against bedwetting in children, to decrease } \\
\text { blood sugar, branches for liver } \\
\text { Utensils and tools - fruit as dye }\end{array}$ \\
\hline \multirow{2}{*}{ Euphorbiaceae } & Euphorbia glaberrima K. Koch. & mdos5s (rdziana) & \\
\hline & Euphorbia iberica Boiss. & mdos6s (rdziana) & \\
\hline \multirow{6}{*}{ Fabaceae } & Anthyllis variegata Boiss. & 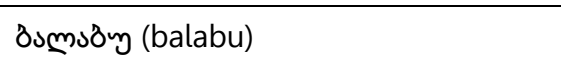 & Animal food - leaves / whole plant grazed and as fodder \\
\hline & Astragalus alpinus $L$. & & \\
\hline & Astragalus capito Boiss. \& Hohen. & & \\
\hline & Astragalus captiosus Boriss. & & \\
\hline & Astragalus denudatus Steven & & \\
\hline & Astragalus supinus C.A. Mey. ex Bunge & & \\
\hline
\end{tabular}


Ethnobotany Research and Applications

\begin{tabular}{|c|c|c|}
\hline Coronilla varia $\mathrm{L}$. & 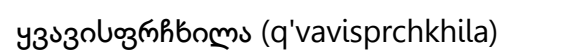 & Food - leaves for khachapuri \\
\hline Galega orientalis Lam. & 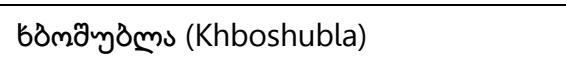 & \\
\hline Lathyrus tuberosus L. & のว๓๓ (telo) & \\
\hline Lotus caucasicus Kuprianova & 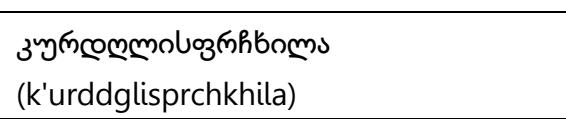 & \\
\hline Medicago falcata L. & 3злолјмо ом6хх (k'viteli iokhja) & Animal food - leaves / whole plant grazed and as fodder \\
\hline Medicago glutinosa M. Bieb. & & Animal food - leaves / whole plant grazed and as fodder \\
\hline Medicago lupulina $\mathrm{L}$. & ззооломо омбхј (k'viteli iokhja) & Animal food - leaves / whole plant grazed and as fodder \\
\hline Melilotus officinalis (L.) Lam. & з3лолјмо dmdm (k'viteli dzodzo) & Animal food - leaves / whole plant grazed and as fodder \\
\hline Onobrychis inermis Steven & 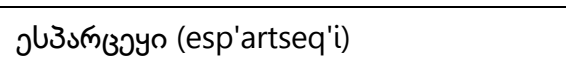 & Animal food - leaves / whole plant grazed and as fodder \\
\hline Onobrychis petraea Desv. & 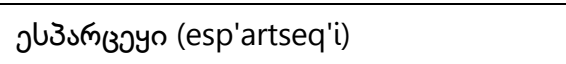 & \\
\hline Orobus cyaneus Steven & 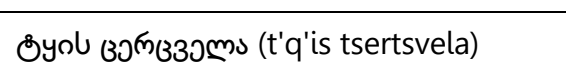 & \\
\hline \multicolumn{3}{|l|}{ Oxytropis albana Steven } \\
\hline \multicolumn{3}{|l|}{ Trifolium alpestre $\mathrm{L}}$. \\
\hline Trifolium ambiguum M. Bieb. & 36moulusaymm (tskhrissamq'uara) & Animal food - leaves / whole plant grazed and as fodder \\
\hline Trifolium arvense $\mathrm{L}$. & 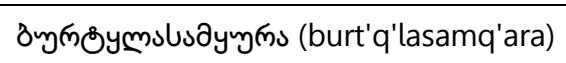 & Animal food - leaves / whole plant grazed and as fodder \\
\hline Trifolium campestre Schreb. & & Animal food - leaves / whole plant grazed and as fodder \\
\hline Trifolium canescens Willd. & 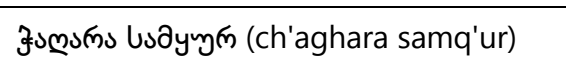 & Animal food - leaves / whole plant grazed and as fodder \\
\hline Trifolium fontanum Bobrov & & Animal food - leaves / whole plant grazed and as fodder \\
\hline Trifolium pratense $\mathrm{L}$. & $\begin{array}{l}\text { Ø̋эosmono bsayyms (ts'urteli } \\
\text { samq'q'ara) }\end{array}$ & Animal food - leaves / whole plant grazed and as fodder \\
\hline Trifolium repens $\mathrm{L}$. & usaymms (samq'ura) & Animal food - leaves as fodder \\
\hline Trifolium rubrum Larrañaga & & Animal food - leaves / whole plant grazed and as fodder \\
\hline Trifolium spadiceum $\mathrm{L}$. & & Animal food - leaves / whole plant grazed and as fodder \\
\hline Trifolium trichocephalum M. Bieb. & 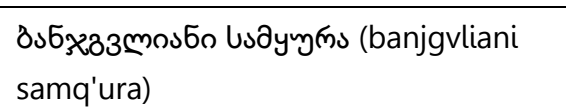 & Animal food - leaves / whole plant grazed and as fodder \\
\hline
\end{tabular}


Ethnobotany Research and Applications

\begin{tabular}{|c|c|c|c|}
\hline & Vicia alpestris Steven & 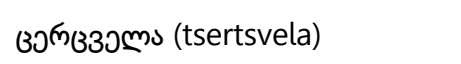 & \\
\hline & Vicia grossheimii Ekytim. & 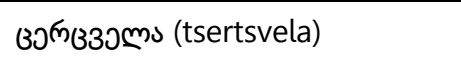 & \\
\hline \multirow{10}{*}{ Gentianaceae } & Gentiana angulosa M. Bieb. & 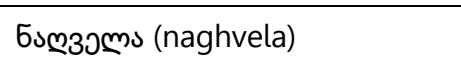 & \\
\hline & Gentiana aquatica $\mathrm{L}$. & б১мзวммs (naghvela) & \\
\hline & Gentiana asclepiadea L. & Б১щзวщмs (naghvela) & Medicinal - leaves cholagogue, gallbladder \\
\hline & Gentiana pumila Jacq. & Б১мзวм๖ (naghvela) & \\
\hline & Gentiana pyrenaica L. & 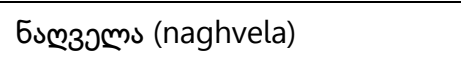 & \\
\hline & Gentiana schistocalyx K. Koch & 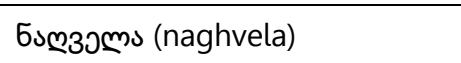 & \\
\hline & Gentiana septemfida Pall. & ৪১రిmyుumson (gabluarai) & Food - leaves for Chave \\
\hline & Gentianella caucasea Holub & & \\
\hline & $\begin{array}{l}\text { Lomatogonium carinthiacum (Wulfen) } \\
\text { Rchb. }\end{array}$ & & \\
\hline & Swertia iberica Fisch. \& C.A. Mey. & 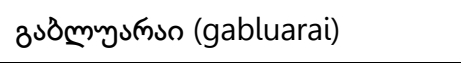 & Food - leaves for Chave \\
\hline \multirow{6}{*}{ Geraniaceae } & Geranium gymnocaulon DC. & & \\
\hline & Geranium ibericum Cav. & 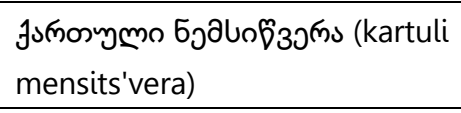 & \\
\hline & $\begin{array}{l}\text { Geranium platypetalum Fisch. \& C.A. } \\
\text { Mey. }\end{array}$ & & \\
\hline & Geranium ruprechtii Grossh. & & \\
\hline & Geranium sanguineum $\mathrm{L}$. & & \\
\hline & Geranium sylvaticum L. & & \\
\hline Hyacinthaceae & Puschkinia scilloides Adams & & \\
\hline \multirow{2}{*}{ Hypericaceae } & Hypericum caucasicum Gorschk. & & \\
\hline & Hypericum nummularioides Trautv. & & \\
\hline
\end{tabular}


Ethnobotany Research and Applications

\begin{tabular}{|c|c|c|c|}
\hline & Hypericum perforatum $\mathrm{L}$. & зঙু১\%১6১ (k'razana) & $\begin{array}{l}\text { Cultural - leaves for perfume } \\
\text { Food - flowers and leaves for tea, and as ingredient in beer } \\
\text { Utensils and tools - leaves and flowers for dye } \\
\text { Medicinal - leaves for gum problems, enuresis, kidneys, nerves, } \\
\text { liver, ulcers, whole plant for gallbladder problems, nerves, oral } \\
\text { inflammation, flowers for nerves, as panacea, for gallbladder } \\
\text { ailments }\end{array}$ \\
\hline \multirow{3}{*}{ Iridaceae } & Crocus scharojanii Rupr. & & \\
\hline & Gladiolus tenuis M. Bieb. & & \\
\hline & Iris pumila L. & 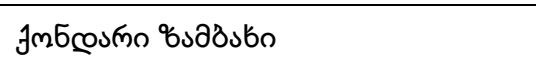 & \\
\hline \multirow{4}{*}{ Juncaceae } & Juncus articulatus L. & łomo (ch'ili) & \\
\hline & Luzula multiflora (Ehrh.) Lej. & summyms (aslura) & \\
\hline & Luzula pseudosudetica V.I. Krecz. & sumymms (aslura) & \\
\hline & Luzula spicata (L.) DC. & summyms (aslura) & \\
\hline \multirow{12}{*}{ Lamiaceae } & Ajuga orientalis L. & & \\
\hline & Betonica macrantha K. Koch & & \\
\hline & Lamium album $\mathrm{L}$. & 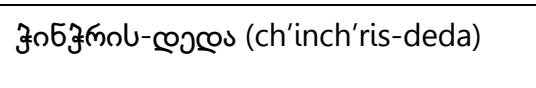 & $\begin{array}{l}\text { Food - whole plant and leaves for phkhali } \\
\text { Medicinal - leaves for hair loss and bath for small children }\end{array}$ \\
\hline & Lamium tomentosum Willd. & & \\
\hline & Mentha longifolia (L.) L.. & 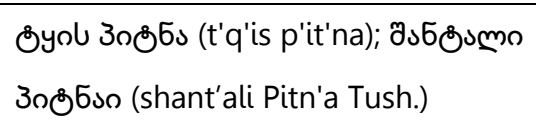 & $\begin{array}{l}\text { Food - leaves for chave, eaten raw, phkhali, tea } \\
\text { Medicinal - leaves for nerves and tea, flowers as panacea }\end{array}$ \\
\hline & Nepeta biebersteiniana Pojark. & & \\
\hline & Nepeta supina Steven & & \\
\hline & Prunella vulgaris $\mathrm{L}$. & & \\
\hline & Salvia tesquicola Klokov \& Pobed. & & \\
\hline & Salvia verticillata $\mathrm{L}$. & cosх๐nূs (dajira) & $\begin{array}{l}\text { Medicinal - anti-inflammatory, enuresis, wounds } \\
\text { Utensils and tools - whole plant as filter }\end{array}$ \\
\hline & Scutellaria leptostegia Juz. & ay6smsços (muzarada) & \\
\hline & Scutellaria oreophila Grossh. & ay६smuços (muzarada) & \\
\hline
\end{tabular}


Ethnobotany Research and Applications

\begin{tabular}{|c|c|c|c|}
\hline & Scutellaria raddeana Juz. & ay\%smıços (muzarada) & \\
\hline & Teucrium nuchense C. Koch & & \\
\hline & Teucrium orientale $\mathrm{L}$. & & \\
\hline & Thymus collinus M. Bieb. & 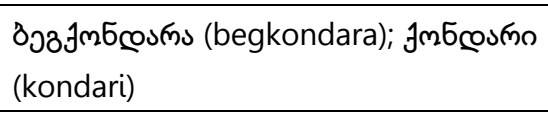 & $\begin{array}{l}\text { Food - leaves as spice and tea } \\
\text { Medicinal - leaves for cough and hypertension }\end{array}$ \\
\hline & Thymus nummularius M. Bieb. & & \\
\hline & Ziziphora puschkinii Adams & 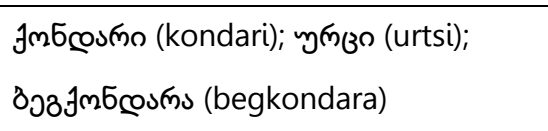 & $\begin{array}{l}\text { Food - leaves as tea } \\
\text { Medicinal - leaves for hypertension, diuretic, antibiotic }\end{array}$ \\
\hline & Fritillaria collina Adams & & \\
\hline Liliaceae & Fritillaria latifolia Willd. & & \\
\hline & Lloydia serotina (L.) Salisb. ex Rchb. & & \\
\hline Linaceae & Linum hypericifolium C. Presl. & & \\
\hline Lycopodiaceae & Lycopodium selago L. & мпзмзмсеоудо & \\
\hline Melanthiaceae & Veratrum lobelianum Bernh. & Ø६sə১ (shkhama) & $\begin{array}{l}\text { Medicinal and veterinary - root and leaves against ektoparasits } \\
\text { and for wounds }\end{array}$ \\
\hline Onagraceae & Chamerion angustifolium (L.) Holub & obsfosmosbsms (thkhatz'arthkhala) & $\begin{array}{l}\text { Food - leaves in khachapuri } \\
\text { Medicinal - leaves as tea }\end{array}$ \\
\hline & Epilobium colchicum Albov & 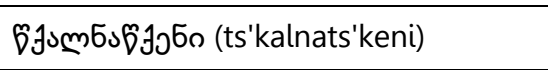 & \\
\hline Ophioglossaceae & Botrychium lunaria (L.) Sw. & 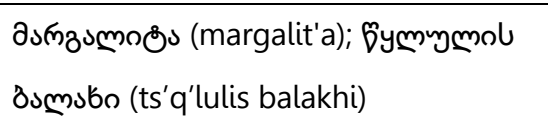 & Medicinal - leaves and whole plant as panacea and for wounds \\
\hline & Cephalanthera longifolia (L.) Fritsch & & \\
\hline & Coeloglossum viride (L.) Hartm. & & \\
\hline & $\begin{array}{l}\text { Dactylorhiza euxina (Nevski) H. } \\
\text { Baumann \& Künkele }\end{array}$ & & \\
\hline Orchidaceae & Goodyera repens (L.) R. Br. & 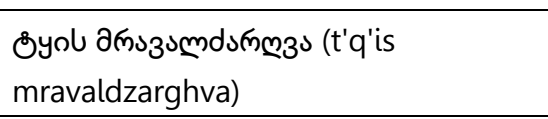 & \\
\hline & Gymnadenia conopsea (L.) R. Br. & & \\
\hline & Herminium monorchis (L.) R. Br. & & \\
\hline
\end{tabular}




\section{Ethnobotany Research and Applications}

\begin{tabular}{|c|c|c|c|}
\hline & $\begin{array}{l}\text { Platanthera chlorantha (Custer) Rchb. } \\
\text { f. }\end{array}$ & mলnozmonm ms (orpotola) & \\
\hline & Traunsteinera sphaerica (Biebl) Schltr. & & \\
\hline \multirow{16}{*}{ Orobanchaceae } & Euphrasia hirtella Jord. ex Reut. & 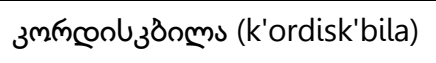 & \\
\hline & Euphrasia minima Jacq. ex DC. & 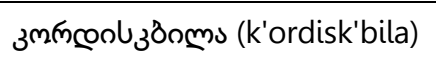 & \\
\hline & Euphrasia pectinata Ten. & 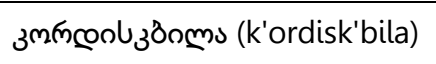 & \\
\hline & Euphrasia petiolaris Wettst. & 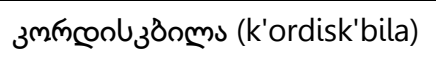 & \\
\hline & Euphrasia tatarica Fisch. ex Spreng. & 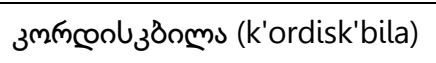 & \\
\hline & Orobanche coerulescens Stephan & 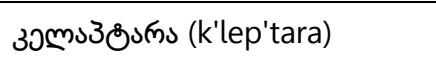 & \\
\hline & Pedicularis armena M. Bieb. & ப১రీกmূs (sat'ilia) & \\
\hline & Pedicularis chroorrhyncha Vved. & U১ఠ̊nmos (sat'ilia) & \\
\hline & Pedicularis comosa L. & ப১ఠ̊nmos (sat'ilia) & \\
\hline & Pedicularis condensata M. Bieb. & U১ఠ̊ంm̄os (sat'ilia) & \\
\hline & Pedicularis crassirostris Bunge & U১ఠீంmos (sat'ilia) & \\
\hline & Pedicularis elisabethae T.N. Popova & U১ఠ̊ంmos (sat'ilia) & \\
\hline & Pedicularis nordmannina Bunge & U১రీంmূంs (sat'ilia) & \\
\hline & Rhinanthus major L. & brnosms (khriala) & \\
\hline & Rhinanthus minor L. & brnosms (khriala) & \\
\hline & $\begin{array}{l}\text { Rhinanthus vernalis (N.W. Zinger) } \\
\text { Schischk. \& Serg. }\end{array}$ & brnosms (khriala) & \\
\hline Oxalidaceae & Oxalis acetosella $\mathrm{L}$. & ةэ১3วms (mzhavela) & Food - leaves eaten raw \\
\hline \multirow{3}{*}{ Papaveraceae } & Corydalis alpestris C.A. Mey. & & \\
\hline & Fumaria schleicheri Soy.-Will. & & \\
\hline & Papaver oreophilum Rupr. & J১J১hм (kakacho) & \\
\hline
\end{tabular}


Ethnobotany Research and Applications

\begin{tabular}{|c|c|c|c|}
\hline Parnassiaceae & Parnassia palustris L. & 3งm5sluyms (p'arnasura) & \\
\hline \multirow{12}{*}{ Plantaginaceae } & Linaria meyeri Kuprian. & Uృmoł̧ (selich'a) & \\
\hline & Plantago atrata Hoppe & & \\
\hline & Plantago lanceolata $\mathrm{L}$. & дm১з১мmdımূ̧১ (mravaldzarghva) & \\
\hline & Plantago major L. & 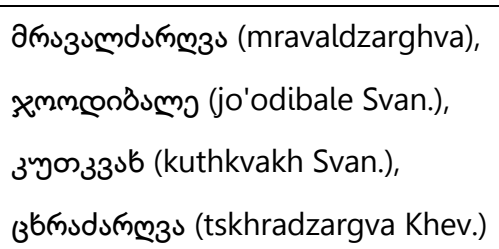 & $\begin{array}{l}\text { Medicinal - leaves for bleeding, cough, digestive system, gastritis, } \\
\text { intestines, wounds; root for stomach problems;; latex for wounds }\end{array}$ \\
\hline & Plantago media L. & & \\
\hline & Veronica caucasica M. Bieb & & \\
\hline & Veronica chamaedrys L. & & \\
\hline & Veronica gentianoides Vahl & & \\
\hline & Veronica minuta C.A. Mey. & & \\
\hline & Veronica peduncularis M. Bieb. & & \\
\hline & Veronica petraea Steven & & \\
\hline & Veronica telephïfolia Vahl & & \\
\hline \multirow{8}{*}{ Poaceae } & Agrostis planifolia K. Koch & 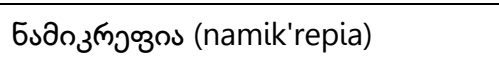 & Animal food - leaves / whole plant grazed and as fodder \\
\hline & Agrostis tenuis Sibth. & 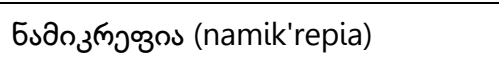 & Animal food - leaves / whole plant grazed and as fodder \\
\hline & Alopecurus dasyanthus Trautv. & ajmsзйcos (melak'uda) & Animal food - leaves / whole plant grazed and as fodder \\
\hline & Alopecurus glacialis K. Koch. & 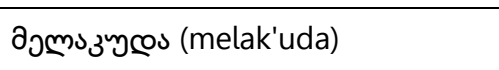 & Animal food - leaves / whole plant grazed and as fodder \\
\hline & $\begin{array}{l}\text { Alopecurus tiflisiensis (G. Westb.) } \\
\text { Grossh. }\end{array}$ & 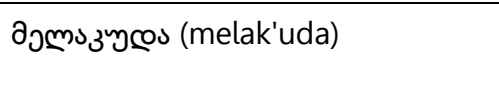 & Animal food - leaves / whole plant grazed and as fodder \\
\hline & $\begin{array}{l}\text { Alopecurus vaginatus (Willd.) Pall. ex } \\
\text { Kunth }\end{array}$ & 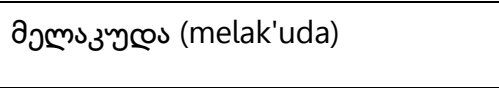 & Animal food - leaves / whole plant grazed and as fodder \\
\hline & $\begin{array}{l}\text { Anthoxanthum alpinum Á. Löve } \& \text { D. } \\
\text { Löve }\end{array}$ & & Animal food - leaves / whole plant grazed and as fodder \\
\hline & Anthoxanthum odoratum $\mathrm{L}$. & 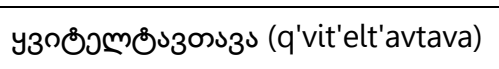 & Animal food - leaves / whole plant grazed and as fodder \\
\hline
\end{tabular}


Ethnobotany Research and Applications

\begin{tabular}{|c|c|c|}
\hline $\begin{array}{l}\text { Arrhenatherum elatius (L.) P. Beauv. ex } \\
\text { J. Presl \& C. Presl }\end{array}$ & 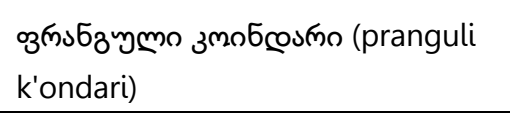 & Animal food - leaves / whole plant grazed and as fodder \\
\hline Avenella flexuosa (L.) Drejer & & Animal food - leaves / whole plant grazed and as fodder \\
\hline Briza marcowiczii Woronow & 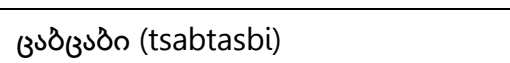 & Animal food - leaves / whole plant grazed and as fodder \\
\hline Briza media L. & 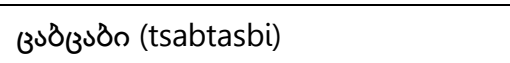 & Animal food - leaves / whole plant grazed and as fodder \\
\hline Briza minor L. & 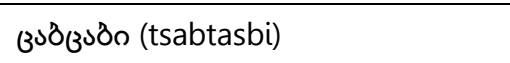 & Animal food - leaves / whole plant grazed and as fodder \\
\hline $\begin{array}{l}\text { Bromopsis biebersteinii (Roem. \& } \\
\text { Schult.) Holub }\end{array}$ & & Animal food - leaves / whole plant grazed and as fodder \\
\hline Bromopsis riparia (Rehmann) Holub & & Animal food - leaves / whole plant grazed and as fodder \\
\hline Bromopsis variegata (M. Bieb.) Holub & & Animal food - leaves / whole plant grazed and as fodder \\
\hline Bromus commutatus Schrad. & & Animal food - leaves / whole plant grazed and as fodder \\
\hline Calamagrostis arundinacea (L.) Roth & ठ๓ndsan (brdzami) & Animal food - leaves / whole plant grazed and as fodder \\
\hline $\begin{array}{l}\text { Calamagrostis pseudophragmites } \\
\text { (Haller f.) Koeler }\end{array}$ & & Animal food - leaves / whole plant grazed and as fodder \\
\hline Catabrosella variegata (Boiss.) Tzvelev & & Animal food - leaves / whole plant grazed and as fodder \\
\hline $\begin{array}{l}\text { Colpodium versicolor(Steven) } \\
\text { Schmalh. }\end{array}$ & & Animal food - leaves / whole plant grazed and as fodder \\
\hline Cynosurus cristatus L. & 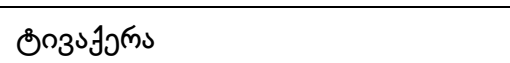 & Animal food - leaves / whole plant grazed and as fodder \\
\hline Dactylis glomerata L. & Usosnosymon (satituri) & Animal food - leaves / whole plant grazed and as fodder \\
\hline Deschampsia cespitosa (L.) P. Beauv. & 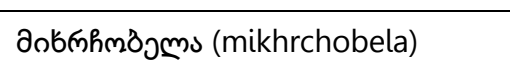 & Animal food - leaves / whole plant grazed and as fodder \\
\hline \multicolumn{3}{|l|}{ Elytrigia gracillima (Nevski) Nevski } \\
\hline \multicolumn{3}{|l|}{ Elytrigia repens (L.) Desv. ex Nevski } \\
\hline Festuca arundinacea Schreb. & & Animal food - leaves / whole plant grazed and as fodder \\
\hline Festuca buschiana (St.-Yves) Tzvelev & & Animal food - leaves / whole plant grazed and as fodder \\
\hline Festuca frigida Grossh. & & Animal food - leaves / whole plant grazed and as fodder \\
\hline Festuca ovina L. & & Animal food - leaves / whole plant grazed and as fodder \\
\hline Festuca pratensis Huds. & ة & Animal food - leaves / whole plant grazed and as fodder \\
\hline Festuca rubra L. & 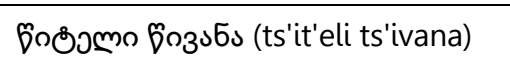 & Animal food - leaves / whole plant grazed and as fodder \\
\hline
\end{tabular}


Ethnobotany Research and Applications

\begin{tabular}{|c|c|c|}
\hline $\begin{array}{l}\text { Festuca ruprechtii (Boiss.) V.I. Krecz. \& } \\
\text { Bobrov }\end{array}$ & & Animal food - leaves / whole plant grazed and as fodder \\
\hline Festuca supina Schur & & Animal food - leaves / whole plant grazed and as fodder \\
\hline Festuca valesiana Schleich. ex Gaudin & & Animal food - leaves / whole plant grazed and as fodder \\
\hline Festuca varia Haenke & & Animal food - leaves / whole plant grazed and as fodder \\
\hline Festuca woronowii Hack. & & Animal food - leaves / whole plant grazed and as fodder \\
\hline $\begin{array}{l}\text { Helictotrichon adzharicum (Albov) } \\
\text { Grossh. }\end{array}$ & & Animal food - leaves / whole plant grazed and as fodder \\
\hline $\begin{array}{l}\text { Helictotrichon asiaticum (Roshev.) } \\
\text { Grossh. }\end{array}$ & & Animal food - leaves / whole plant grazed and as fodder \\
\hline Helictotrichon pubescens (Huds.) Pilg. & & Animal food - leaves / whole plant grazed and as fodder \\
\hline Hordeum violaceum Boiss. \& Hohen. & Jコmo (queri) & Medicinal - leaves to treat cancer \\
\hline \multicolumn{3}{|l|}{$\begin{array}{l}\text { Kobresia capillifolia (Decne.) C.B. } \\
\text { Clarke }\end{array}$} \\
\hline $\begin{array}{l}\text { Koeleria caucasica (Trin. ex Domin) B. } \\
\text { Fedtsch. }\end{array}$ & 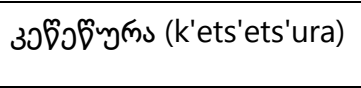 & \\
\hline Koeleria luerssenii Domin & 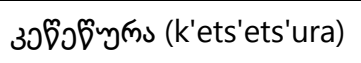 & \\
\hline Koeleria macrantha (Ledeb.) Schult & 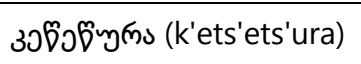 & \\
\hline \multicolumn{3}{|l|}{ Melica transsilvanica Schur. } \\
\hline Milium effusum $\mathrm{L}$. & & Animal food - leaves / whole plant grazed and as fodder \\
\hline Nardus stricta L. & doз3s (dzigva) & \\
\hline Phleum alpinum $\mathrm{L}$. & & Animal food - leaves / whole plant grazed and as fodder \\
\hline Phleum montanum K. Koch & & Animal food - leaves / whole plant grazed and as fodder \\
\hline Phleum phleoides (L.) H. Karst. & & Animal food - leaves / whole plant grazed and as fodder \\
\hline Phleum pratense $\mathrm{L}$. & 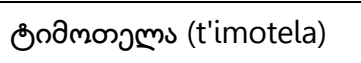 & Animal food - leaves / whole plant grazed and as fodder \\
\hline $\begin{array}{l}\text { Phragmites australis (Cav.) Trin. ex } \\
\text { Steud. }\end{array}$ & momo (leli) & Animal food - leaves / whole plant grazed and as fodder \\
\hline Poa alpina $\mathrm{L}$. & 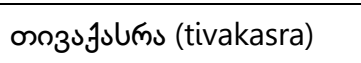 & Animal food - leaves / whole plant grazed and as fodder \\
\hline Poa badensis Haenke ex Willd. & 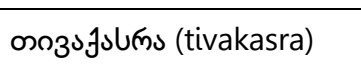 & Animal food - leaves / whole plant grazed and as fodder \\
\hline Poa caucasica Trin. & ๓оз১j১ums (tivakasra) & Animal food - leaves / whole plant grazed and as fodder \\
\hline
\end{tabular}


Ethnobotany Research and Applications

\begin{tabular}{|c|c|c|c|}
\hline & Poa compressa L. & 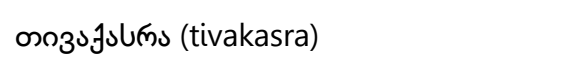 & Animal food - leaves / whole plant grazed and as fodder \\
\hline & Poa nemoralis L. & 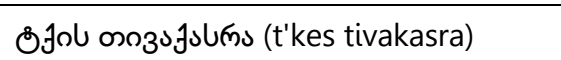 & Animal food - leaves / whole plant grazed and as fodder \\
\hline & Poa pratensis $\mathrm{L}$. & 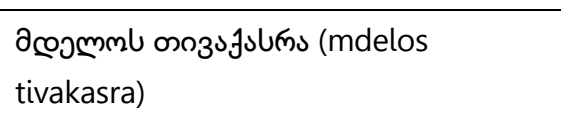 & Animal food - leaves / whole plant grazed and as fodder \\
\hline & Trisetum buschianum Seredin & & Animal food - leaves / whole plant grazed and as fodder \\
\hline & Trisetum flavescens (L.) P. Beauv. & 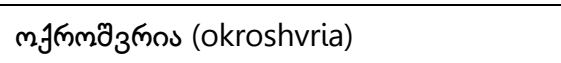 & Animal food - leaves / whole plant grazed and as fodder \\
\hline & $\begin{array}{l}\text { Trisetum rigidum (M. Bieb.) Roem. \& } \\
\text { Schult. }\end{array}$ & & Animal food - leaves / whole plant grazed and as fodder \\
\hline \multirow{3}{*}{ Polygalaceae } & Polygala alpicola Rupr. & 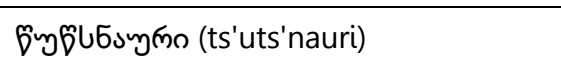 & \\
\hline & Polygala caucasica Rupr. & 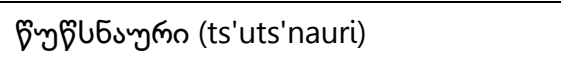 & \\
\hline & Polygala transcaucasica Tamamsch. & 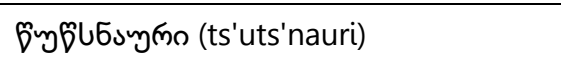 & \\
\hline \multirow{6}{*}{ Polygonaceae } & Polygonum alpestre C.A. Mey. & & \\
\hline & Polygonum alpinum All. & 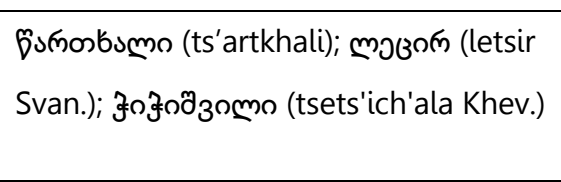 & $\begin{array}{l}\text { Food - leaves in khacahpuri, phkhali, sats'ebai and eaten raw, } \\
\text { stems for phkhali and pickled } \\
\text { Medicinal - leaves for gastrointestinal problems and joints } \\
\text { Utensils and tools - root as dye }\end{array}$ \\
\hline & Polygonum carneum K. Koch & 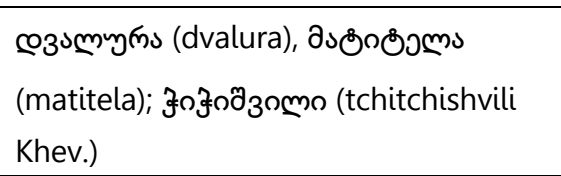 & $\begin{array}{l}\text { Medicinal - root for cirrhosis, diarrhea, liver, lungs, flower for } \\
\text { lungs }\end{array}$ \\
\hline & Polygonum viviparum $\mathrm{L}$. & & \\
\hline & Rumex acetosa $\mathrm{L}$. & 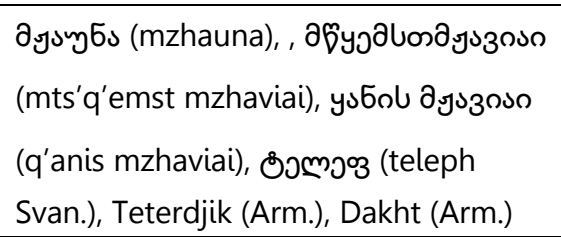 & $\begin{array}{l}\text { Food - leaves for khachapuri, eaten raw, phkhali, sats'ebai, } \\
\text { pickled, stems eaten raw, pickled }\end{array}$ \\
\hline & Rumex acetosella $\mathrm{L}$. & aงsubs (mzhauna) & Food - leaves in khachapuri, eaten raw, pickled, phkhali \\
\hline
\end{tabular}


Ethnobotany Research and Applications

\begin{tabular}{|c|c|c|c|}
\hline & Rumex alpinus $\mathrm{L}$. & 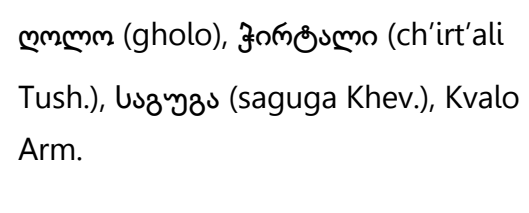 & $\begin{array}{l}\text { Food - leaves in chave, phkhali, pickled, stems pickled and in } \\
\text { phkhali } \\
\text { Medicinal - seeds for intestinal problems, hemorrhoids, colitis, } \\
\text { leaves to treat tumors } \\
\text { Utensils and tools - root for dye }\end{array}$ \\
\hline & Rumex scutatus L. & 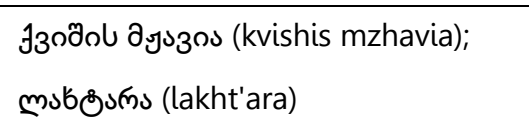 & Food - leaves for phkhali and eaten raw, stems pickled \\
\hline \multirow{7}{*}{ Primulaceae } & Androsace albana Steven & & \\
\hline & Androsace barbulata Ovcz. & & \\
\hline & Androsace villosa $\mathrm{L}$. & & \\
\hline & Lysimachia verticillaris Spreng. & 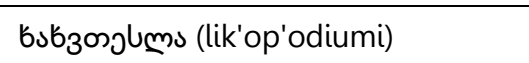 & \\
\hline & Primula algida Adams & & Food - leaves for phkhali \\
\hline & Primula amoena M. Bieb. & & Food - leaves for phkhali \\
\hline & Primula auriculata Lam. & & Food - leaves for phkhali \\
\hline \multirow{13}{*}{ Ranunculaceae } & Aconitum anthora L. & 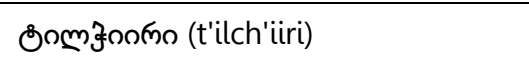 & \\
\hline & Aconitum nasutum Fisch. ex Rchb. & 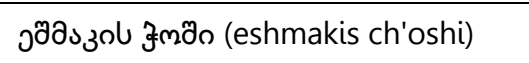 & \\
\hline & Anemonastrum fasciculatum (L.) Holub & & \\
\hline & Anemone speciosa Adam ex G. Pritz. & & \\
\hline & Delphinium caucasicum C.A. Mey. & & \\
\hline & Pulsatilla violacea Rupr. & 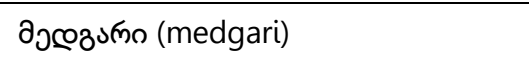 & \\
\hline & Ranunculus acutilobus Ledeb. & Buss (baia) & \\
\hline & $\begin{array}{l}\text { Ranunculus astrantiifolius Boiss. ex } \\
\text { Trautv. }\end{array}$ & Juss (baia) & \\
\hline & Ranunculus baidarae Rupr. & Juss (baia) & \\
\hline & Ranunculus buhsei Boiss. & Boss (baia) & \\
\hline & Ranunculus caucasicus M. Bieb. & Boss (baia) & \\
\hline & Ranunculus grandiflorus $\mathrm{L}$. & Buss (baia) & \\
\hline & Ranunculus lojkae Sommier \& Levier & Bsos (baia) & \\
\hline
\end{tabular}


Ethnobotany Research and Applications

\begin{tabular}{|c|c|c|c|}
\hline & Ranunculus oreophilus M. Bieb. & Bsos (baia) & \\
\hline & Ranunculus repens $\mathrm{L}$. & Bsos (baia) & \\
\hline & Thalictrum alpinum $\mathrm{L}$. & & \\
\hline & Thalictrum bushianum Kem.-Nath. & & \\
\hline & Thalictrum flavum $\mathrm{L}$. & & \\
\hline & Thalictrum foetidum $\mathrm{L}$. & ৬১ప১ఠీmృ (samat'le) & \\
\hline & Trollius ranunculinus (Sm.) Stearn & & \\
\hline Resedaceae & Reseda lutea $\mathrm{L}$. & 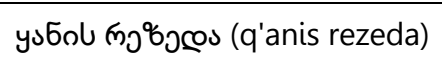 & \\
\hline \multirow{16}{*}{ Rosaceae } & Alchemilla caucasica Buser & 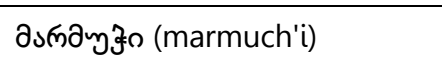 & Medicinal - leaves \\
\hline & Alchemilla chlorosericea Juz. & 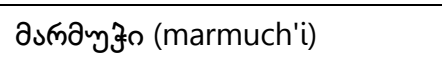 & Medicinal - leaves \\
\hline & Alchemilla debilis Juz. & 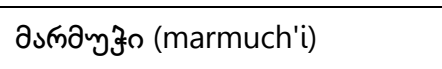 & Medicinal - leaves \\
\hline & Alchemilla dura Buser & 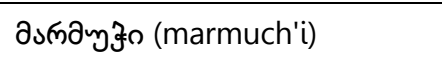 & Medicinal - leaves \\
\hline & Alchemilla elisabethae Juz. & 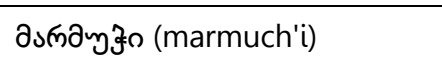 & Medicinal - leaves \\
\hline & Alchemilla glabricaulis $\mathrm{H}$. Lindb. & 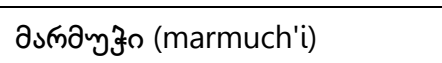 & Medicinal - leaves \\
\hline & Alchemilla laeta Juz. & 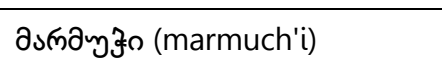 & Medicinal - leaves \\
\hline & Alchemilla languida Buser & 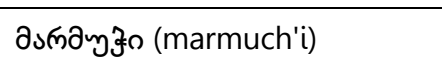 & Medicinal - leaves \\
\hline & Alchemilla retinervis Buser & 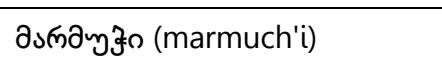 & Medicinal - leaves \\
\hline & Alchemilla rigida Buser & 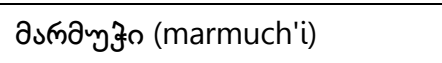 & Medicinal - leaves \\
\hline & Alchemilla sericata Reichenb. ex Bus. & 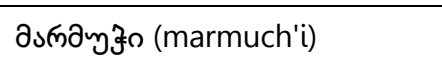 & Medicinal - leaves \\
\hline & Alchemilla sericea Willd. & 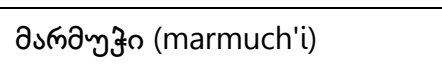 & Medicinal - leaves \\
\hline & Alchemilla valdehirsuta Buser & 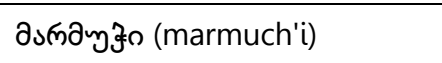 & Medicinal - leaves \\
\hline & Cotoneaster integerrimus Medik. & & \\
\hline & Cotoneaster laxiflorus Jacq. ex Lindl. & & \\
\hline & Dryas caucasica Juz. & çmosçon (driadi) & \\
\hline
\end{tabular}


Ethnobotany Research and Applications

\begin{tabular}{|c|c|c|}
\hline \multicolumn{3}{|l|}{$\begin{array}{l}\text { Potentilla adscharica Sommier \& } \\
\text { Levier }\end{array}$} \\
\hline $\begin{array}{l}\text { Potentilla crantzii (Crantz) Beck ex } \\
\text { Fritsch }\end{array}$ & & \\
\hline \multicolumn{3}{|l|}{ Potentilla erecta (L.) Raeusch. } \\
\hline \multicolumn{3}{|l|}{ Potentilla gelida C.A. Mey. } \\
\hline \multicolumn{3}{|l|}{ Potentilla recta $\mathrm{L}}$. \\
\hline Potentilla reptans $\mathrm{L}$. & 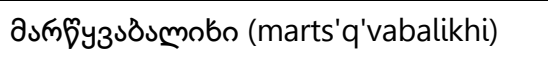 & \\
\hline Rosa oxyodon Boiss. & 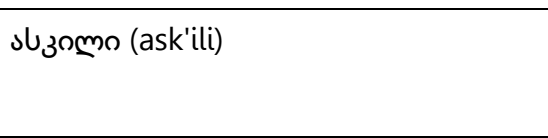 & $\begin{array}{l}\text { Food - fruit and flower for alcohol, also eaten raw, fruit and leaves } \\
\text { as tea } \\
\text { Medicinal - fruit for colds, cough, gallbladder, kidneys and as tea }\end{array}$ \\
\hline Rosa pulverulenta M. Bieb. & د৬зомо (ask'ili) & $\begin{array}{l}\text { Food - fruit and flower for alcohol, also eaten raw, fruit and leaves } \\
\text { as tea } \\
\text { Medicinal - fruit for colds, cough, gallbladder, kidneys and as tea }\end{array}$ \\
\hline Rubus idaeus $\mathrm{L}$. & 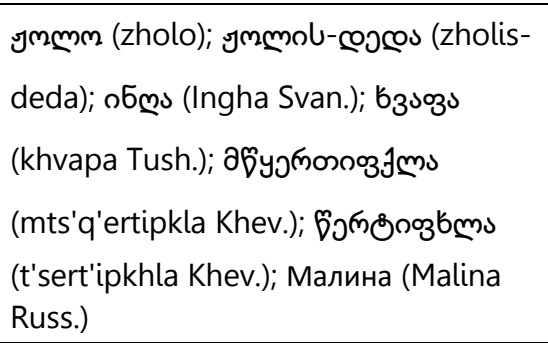 & $\begin{array}{l}\text { Food - fruits and flowers eaten raw, fruits for chave, leaves for tea } \\
\text { Medicinal - leaves for colds and tea, fruits for wounds }\end{array}$ \\
\hline Sanguisorba officinalis L. & 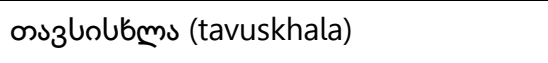 & \\
\hline Sibbaldia parviflora Willd. & ૧ృ & \\
\hline Sibbaldia semiglabra C.A. Mey. & 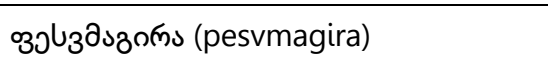 & \\
\hline Sorbus caucasigena Kom. ex Gatsch. & 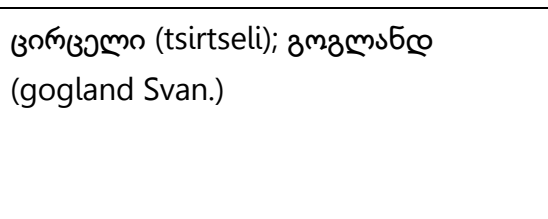 & $\begin{array}{l}\text { Food - fruits for alcohol, beer, eaten raw, marmalade } \\
\text { Medicinal - fruits for blood pressure, heart, hypertension, wounds, } \\
\text { leaves for cramps } \\
\text { Utensils and tools - stem for household utensils, tool handles, } \\
\text { brooms }\end{array}$ \\
\hline Spiraea hypericifolia L. & 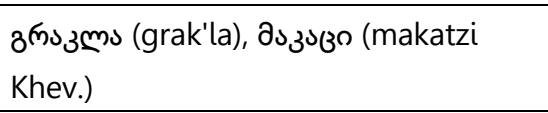 & Utensils and Tools - stems as brooms \\
\hline
\end{tabular}


Ethnobotany Research and Applications

\begin{tabular}{|c|c|c|c|}
\hline \multirow{10}{*}{ Rubiaceae } & Asperula albovii Manden. & & \\
\hline & Asperula cristata V.I. Krecz. & & \\
\hline & Cruciata glabra Ehrend. & & Animal food - leaves / whole plant grazed and as fodder \\
\hline & Cruciata laevipes Opiz & & Animal food - leaves / whole plant grazed and as fodder \\
\hline & Galium galioides Soó & & \\
\hline & Galium lucidum All. & & \\
\hline & Galium mollugo L. & & \\
\hline & Galium spurium L. & & \\
\hline & Galium valantioides M. Bieb. & & \\
\hline & Galium verum $\mathrm{L}$. & 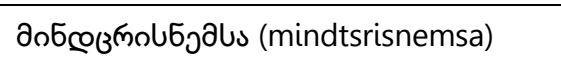 & \\
\hline \multirow[t]{2}{*}{ Salicaceae } & Salix caprea L. & 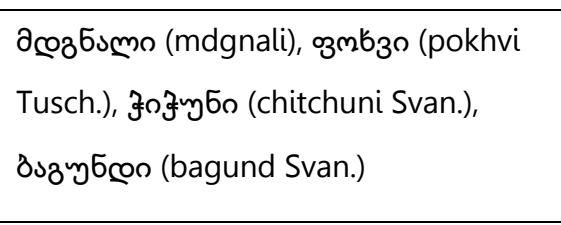 & $\begin{array}{l}\text { Animal Food - leaves as fodder } \\
\text { Construction - stems and branches as timber, walls, fences } \\
\text { Medicinal - leaves and bark for arthritis, gallstones, kidneys } \\
\text { Utensils and tools - stem for tool handles, bopws, snowshovels, } \\
\text { baskets, tough utensils }\end{array}$ \\
\hline & Salix kazbekensis A.K. Skvortsov & & \\
\hline \multirow{2}{*}{ Santalaceae } & Thesium laxiflorum Trautv. & & \\
\hline & Thesium procumbens C.A. Mey. & & \\
\hline \multirow{9}{*}{ Saxifragaceae } & Saxifraga cartilaginea Willd. & 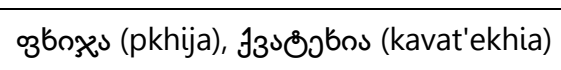 & \\
\hline & Saxifraga cymbalaria L. & 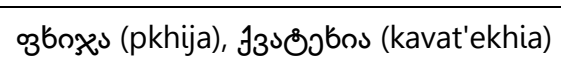 & \\
\hline & Saxifraga exarata Vill. & 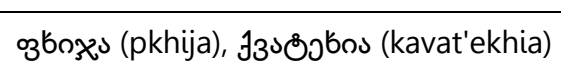 & \\
\hline & Saxifraga flagellaris Willd. & 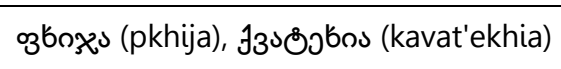 & \\
\hline & Saxifraga juniperifolia Adams & 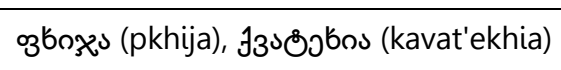 & \\
\hline & Saxifraga kolenatiana Regel & 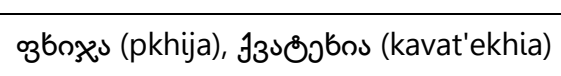 & \\
\hline & Saxifraga moschata Wulfen & 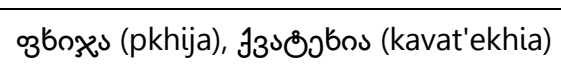 & \\
\hline & Saxifraga ruprechtiana Manden. & 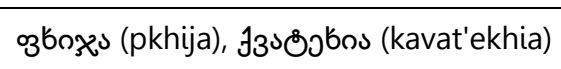 & \\
\hline & Saxifraga sibirica L. & 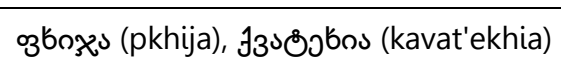 & \\
\hline \multirow{2}{*}{ Scrophulariaceae } & Scrophularia minima M. Bieb. & & \\
\hline & Scrophularia olympica Boiss. & & \\
\hline
\end{tabular}


Ethnobotany Research and Applications

\begin{tabular}{|c|c|c|c|}
\hline & Scrophularia sp. & & \\
\hline & Scrophularia variegata M. Bieb. & & \\
\hline & Verbascum gossypinum M. Bieb. & 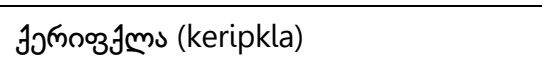 & \\
\hline & Verbascum phoeniceum L. & 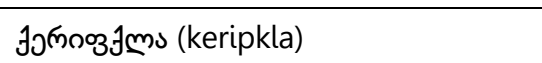 & \\
\hline & Verbascum pyramidatum M. Bieb. & 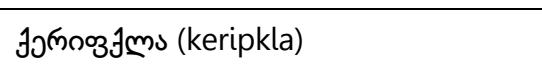 & \\
\hline & Verbascum sp. & 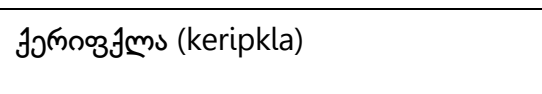 & $\begin{array}{l}\text { Medicinal - leaves for gallbladder, hemorrhoids, kidneys, skin } \\
\text { problems }\end{array}$ \\
\hline Selaginellaceae & Selaginella helvetica (L.) Link. & & \\
\hline Solanaceae & Solanum persicum Roem. \& Schult. & 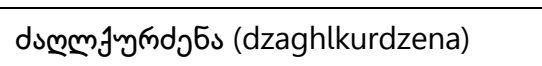 & \\
\hline & Solanum pseudopersicum Pojark. & & \\
\hline Thymelaeaceae & Daphne glomerata Lam. & 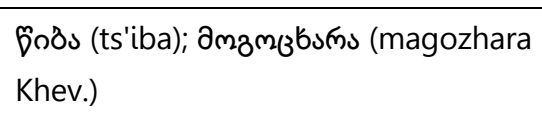 & Medicinal - leaves for toothache \\
\hline Trilliaceae & Paris quadrifolia $\mathrm{L}$. & bsmolonzums (kharistvala) & \\
\hline & Parietaria judaica L. & 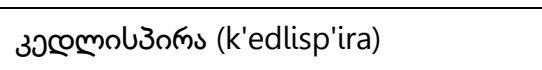 & \\
\hline Urticaceae & Urtica dioica $\mathrm{L}$. & 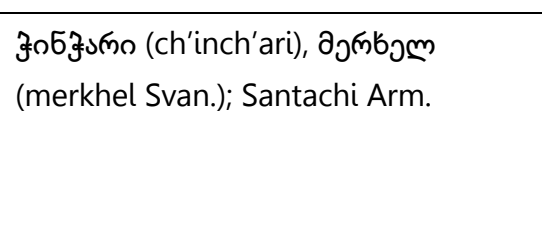 & $\begin{array}{l}\text { Animal food - leaves fodder } \\
\text { Food - leaves and stems as beverage, khachapuri, khinkali, } \\
\text { phkhali, pickled } \\
\text { Medicinal - leaves for burns, joint pain, nerves, increase of } \\
\text { hemoglobin; stem for burns }\end{array}$ \\
\hline & Viola odorata $\mathrm{L}$. & os (ia); os-os (ia ia) & Food - root pickled \\
\hline & Viola oreades M. Bieb. & os (ia); os-os (ia ia) & Food - root pickled \\
\hline Vtolaceae & Viola somchetica C. Koch & os (ia); o১-os (ia ia) & Food - root pickled \\
\hline & Viola $s p$. & os (ia); os-os (ia ia) & Food - root pickled \\
\hline
\end{tabular}

Chave: made of dried herbs by boiling them, adding flour, fat (with or without meat) and salt; Mkhlovana: bread filled with beetroot leaves, spinach, herbs; Khachapuri: bread filled with cheese and herbs; Khinkali: dumplings with herbs and meat; Phkhali: minced herbs, sometimes mixed with walnuts, eaten as spread or cooked in pie; Sats'ebai: fresh herbs dipped in sour milk 
Georgia counts as one of the oldest Christian regions, adopting Christianity around 320 CE. A great example for early church construction is Gergeti Trinity Church, built in the $14^{\text {th }}$ century, located at $2170 \mathrm{~m}$ at the base of Mount Kazbeghi $(5047 \mathrm{~m})$, overlooking the narrow valley leading from Georgia to Ingushetia. However, ancestral shrines are still very common in many regions of Georgia.

Grapes - Vitis vinifera L. (Vitaceae) show genetic diversity in Georgia, with about 500 varieties known (Javakhishvili 1987, Ketskhoveli et al. 1960, Ramishvili 1988, This et al. 2006), and in most regions the population takes great pride to produce their own wine and share it with visitors. Hardly any house in the Georgian lowlands is without at least some grapes in its garden or backyard. Today, forty-one cultivars of grapevine are used as commercial varieties in Georgia (Bedoshvili 2010), and good wine is readily available, but the history of grape cultivation and winemaking goes back millennia. Like in other parts of Europe, Georgian grapes were devastated by the Phylloxera vastatrix (Planchon) Signoret and after the infestation in the 1860 s most Georgian grape varieties are now grafted on rootstocks of American grapes resistant to Phylloxera.

Wheat - Triticum L. (Poaceae): In the 1940s sixteen species, 144 varieties, and 150 forms of wheat were registered in Georgia (Menabde 1948.This diversity has however greatly diminished, and most species had already disappeared by the 1960s, when introduced cultivars were favored in Soviet kolkhoz systems. At present, none of these species are sown in Georgian commercial agriculture. Pistrick et al. 2009) report some traditional varieties of bread wheat in Tusheti, Meskheti, Javakheti and Svaneti

Barley - Hordeum vulgare L. (Poaceae) is also an ancient agricultural crop in Georgia, and had particular importance in beer production, as well a function in religious rituals and traditional medicine (Badr et al. 2000, Javakhishvili 1987).

Caucasian Rye - Secale cereale L. (Poaceae) used to be cultivated in the high mountain regions of Georgia (1800$2200 \mathrm{~m}$ ), and entered into bread and beer production, although barley was preferred for beer.

Legumes, especially peas (Pisum sativum L.), lentils (Lens cornicularis L.), chickpeas (Cicer arietinum $\mathrm{L}$.), faba beans (Vicia faba L.) are still commonly grown in home gardens, and Green Pea (Pisum sativum) is thought to have originated in the Southern Caucasus. Traditional vegetables like garden lettuce (Lactuca sativa L.), beans (Phaseolus vulgaris L.), sweet basil (Ocimum basilicum L.), peppermint (Mentha x piperita L.), onions (Allium cepa L.), sugar beets (Beta vulgaris L.), spinach (Spinaca oleracea L.), carrots (Daucus carota L.), radishes (Raphanus sativus L.), turnips (Brassica rapa var. rapa L.), Welsh onion (Allium fistulosum L.), Amaranth (Amaranthus viridis L.), Goosefoot (Chenopodium album L.), leeks (Allium apeloprasum L.) and garlic (Allium sativum L.) are still very common throughout the region, and herbs like parsley (Petroselinum crispum (Mill.) Fuss.), coriander (Coriandrum sativum L.), tarragon (Artemisia dracunculus L.), savory (Satureja hortensis L.), gardencress (Lepidium sativum L.), dill (Anethum graveolens L.), fennel (Foeniculum vulgare Mill.), celery (Apium dulce Mill.), Allium fistulosum L., Brassica rapa L. subsp. rapifera Metzger, Lathyrus sativus L., Linum usitatissimum L., Medicago sativa L., Onobrychis transcaucasica Grossh., Pisum arvense L., Trigonella caerulea (L.) Ser. are cultivated almost everywhere. In addition, introduced species like zucchini (Cucurbita pepo L.), cucumber (Cucumis sativus L.), eggplant (Solanum melongena L.), marigold (Tagetes patula L.), watermelon (Citrullus lanatus (Thunb.) Matsum., Nakai), sunflower (Helianthus annuus L.), tomato (Solanum lycopersicum (Mill.) Wettst.), pepper (Capsicum annuum L.), potato (Solanum tuberosum L.), and maize (Zea mays L.), and were found to be popular ingredients of local cuisine. A large number of additional species is traditionally also grown in home gardens, e.g., Sour plum (Prunus cerasifera var. divaricata) is commonly used as sauce with meat, Rose hips (Rosa canina L.) are often used for tea and to make jam, and Staphyllea pinnata L. (Bladdernut) inflorescences are a favorite pickle.

\section{Pkhali and Pickles - emblematic foods of the Caucasus}

Of all food preparations the use of plants as ingredient of boiled herb preparations (mostly as 3 s $^{\circ}$ sozbymol o̧bsmo - gazapkhuli pkhali = Spring Pkhkali, as the first vitamin source after winter), and as lacto-fermented or vinegar-based pickles are probably the most emblematic ones in the Caucasus. Only $60 \%$ of participants reported making pickles / lacto fermented preparations. Of these, over $16 \%$ each came from Zemo Imereti and Khevsureti, and $12 \%$ each from Zvemo Svaneti, the Javakheti-Plateau, representing all high altitude - short growing season areas, where the population does need to preserve food for winter. In the case of Pkhali, over $93 \%$ of all participants 
reported to use such boiled herbs, normally in Spring. Zemo Imereti (19\% of all Phkhali preparations), Tori and Kvemo Racha (16\% each), Tusheti (15\%) and Khevsureti (14\%) - all mountain regions with long winters, stood out as the real "herb eater" areas. In contrast to the pickled species, essentially only young leaves were used for pkhali, with great emphasis on the same families indicated in pickles. (all pickled plant species were also used for phkhali). The elaboration of phkhali often involves many steps to reduce the toxicity of species used, and in most cases a wide variety of herbs are included in each preparation.

\section{Subalpine and Alpine Vegetation communities Species Area Curve analysis}

A total of 563 plant species were recorded from 619 plots in alpine pastures of Caucasus. The species area curve analysis revealed that the maximum number of plant species appeared up to 600 plots after which the species curve became parallel, as no new species were recorded further. We also found that the curve reached the asymptote, showing adequate sampling in the targeted region (Figure 1).

\section{Species Area Curve}

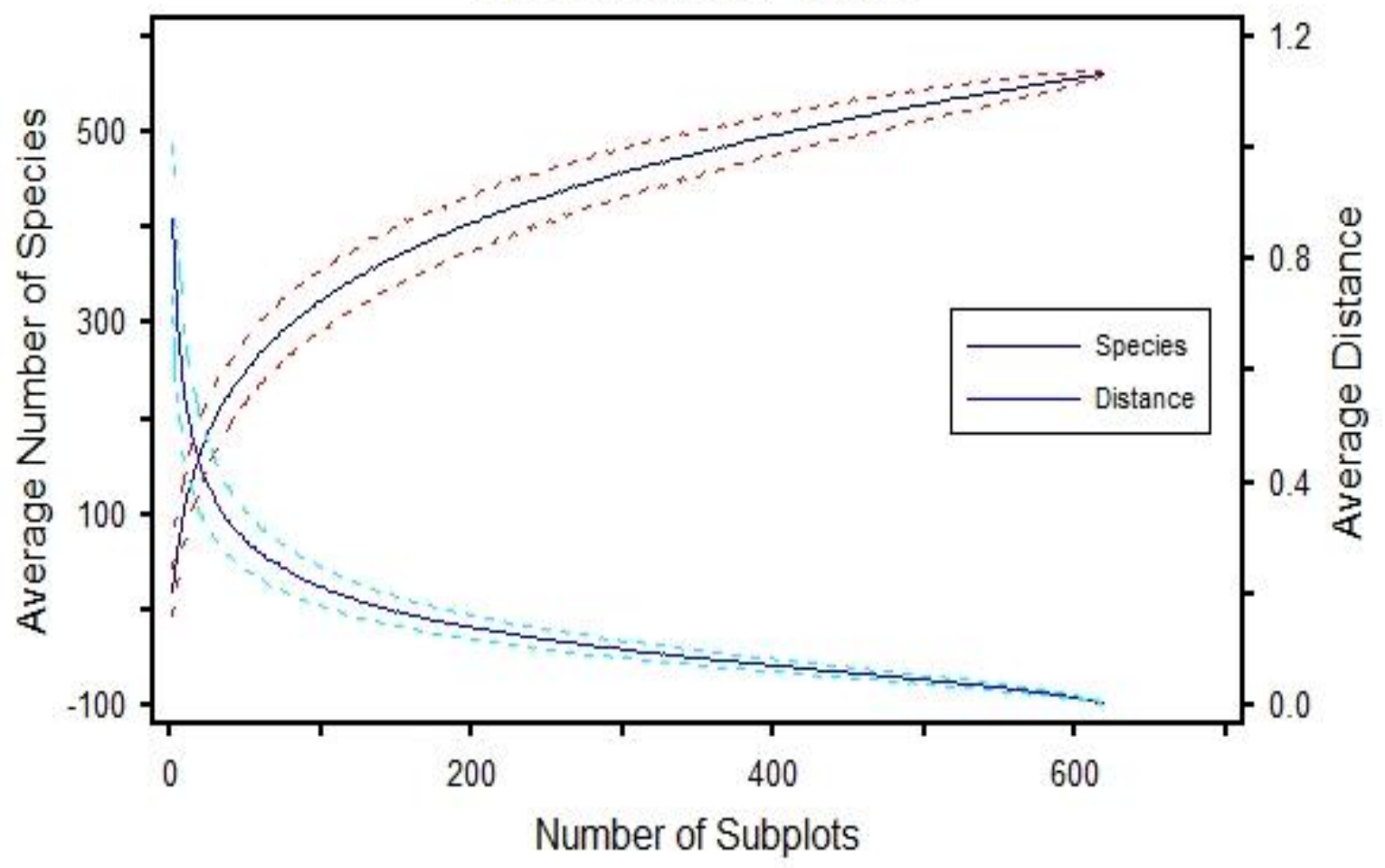

Figure 1. Species Area Curve illustrating the adequacy level of sampling sites (619) for the vegetation (563 plant species) of the study area.

\section{Classification of the alpine vegetation TWINSPAN}

According to TWINSPAN, a total of seven major plant communities were recognized by clustering all the plant species (563 plant species) recorded in 619 plots under the strong influence of altitude and exposition variables (TWINSPAN, Figure 2). They ranged from alpine ranges of $1735 \mathrm{~m}$ to the cold desert $(3600 \mathrm{~m}$. We can observe by the TWINSPAN method, two large different clusters, which show a high cluster heterogeneity value (Lambda $=0.5568$.) One of these clusters had four major plant communities and was formed by 591 plots, while the other cluster presented three major plant communities structured in 28 plots. Furthermore, we observed different subdivisions within these two large groups. Each community was composed of different indicator species and was recorded at different altitudinal range (Figure 2). 


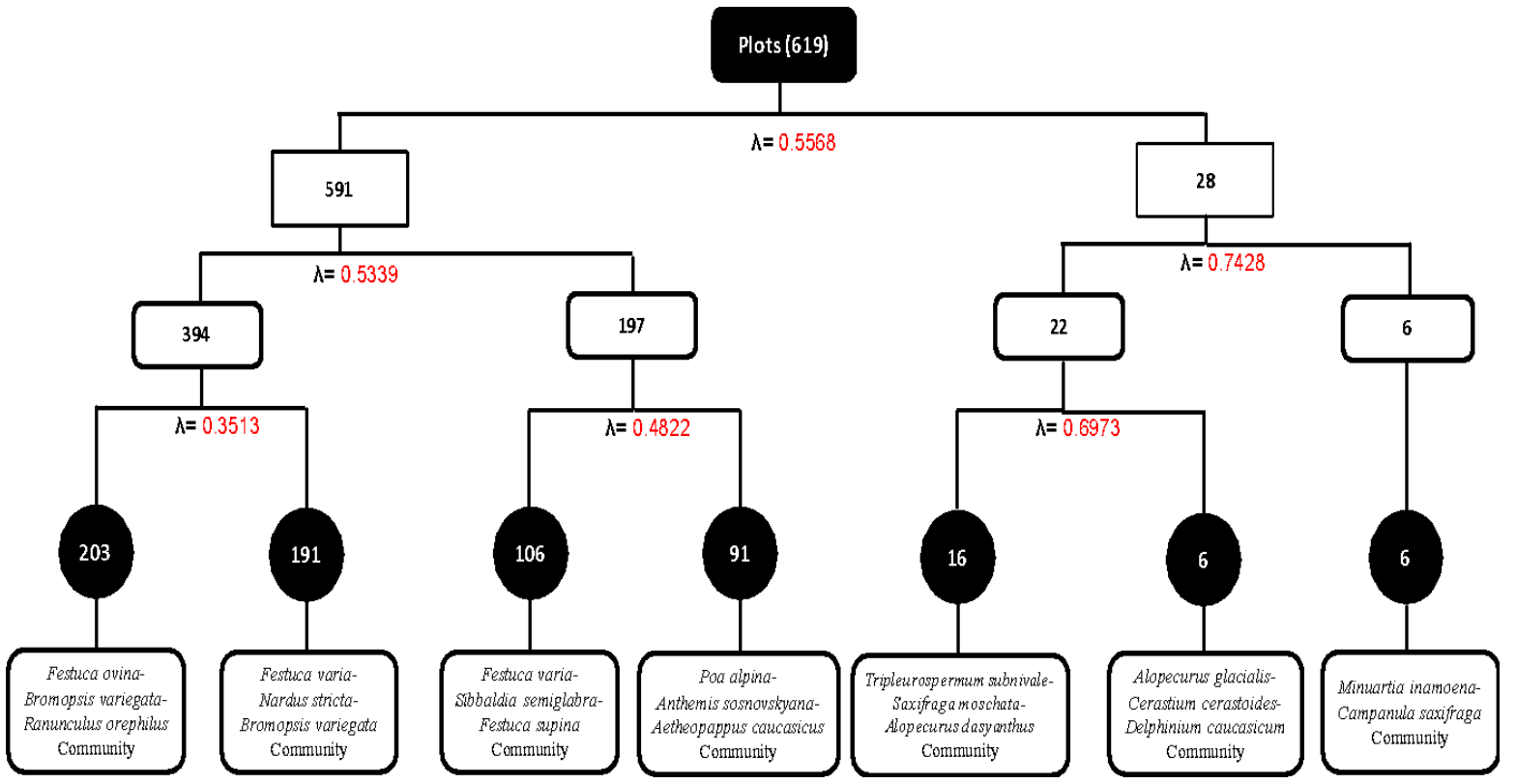

Figure 2: TWINSPAN classification of the alpine vegetation of the Georgian Caucasus. For the classification of 563 plant species and 619 plots simultaneously.

\section{Altitude}

We observed a significant variation in the average altitude between plant communities $\left(x^{2}=3905.4, \mathrm{df}=6, \mathrm{p}<0.001\right.$, Figures 3a,b,c). FBR (FBR: Festuca ovina, Bromopsis variegata, Ranunculus oreophilus) and FNB (FNB: Festuca varia, Nardus stricta, Bromopsis variegata) had the lowest values of average altitude, $1954 \pm 9.9$ and $2255 \pm 17$ (mean \pm SE) respectively, significantly different from each other and from all other communities. ACD (ACD: Alopecurus glacialis Cerastium cerastioides, Delphinium caucasicum), FSF (FSF: Festuca varia, Sibbaldia, semiglabra Festuca supina), MC (MC: Minuartia inamoena, Campanula saxifraga) and PAA (PAA: Poa alpina, Anthemis sosnovskyana, Aetheopappus caucasicus (TSA: Tripleurospermum subnivale, Saxifraga moschata, Alopecurus dasyanthus) showed intermediate values of average altitude $(3125 \pm 25,2952 \pm 15,2903 \pm 21,2897 \pm 2$, respectively), and TSA $(3213 \pm 49)$ had the highest average altitude.

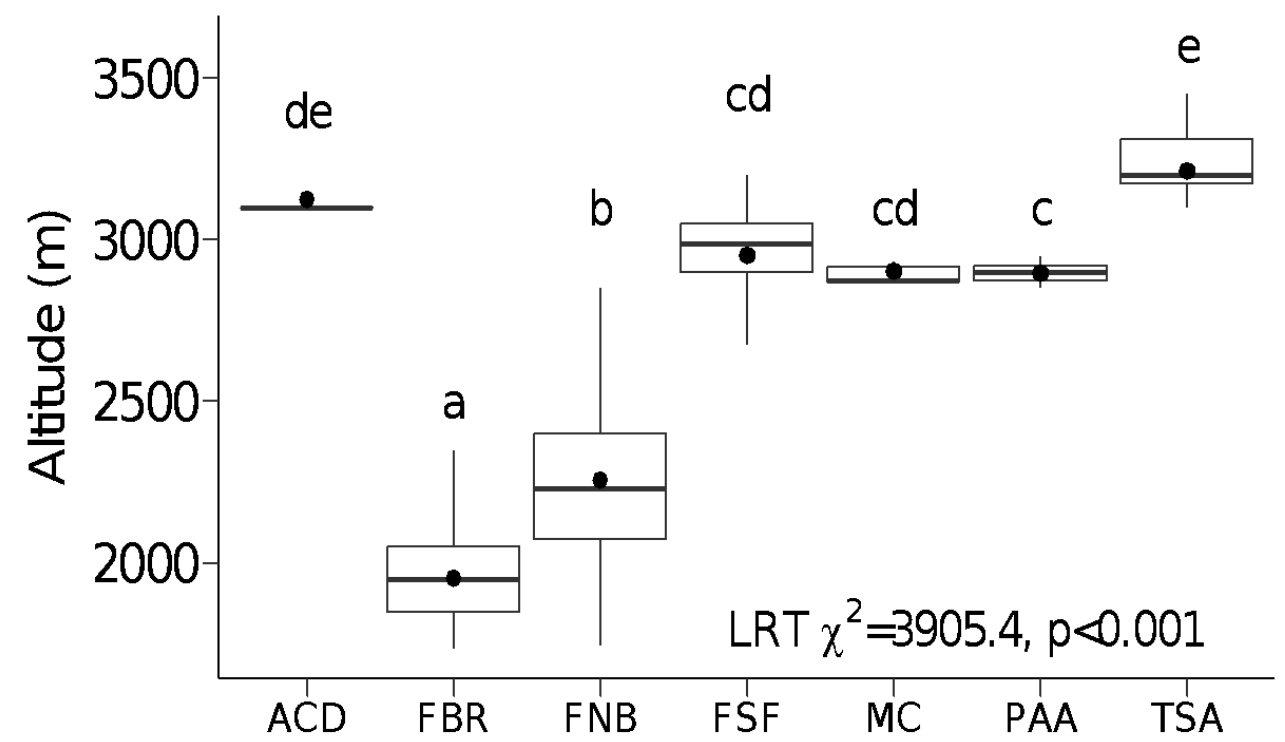

Figure 3a. Variation in the average altitude between plant communities. Letters differ from each other by the estimated marginal means. Box plots are represented by raw data, median and mean (black dot). 


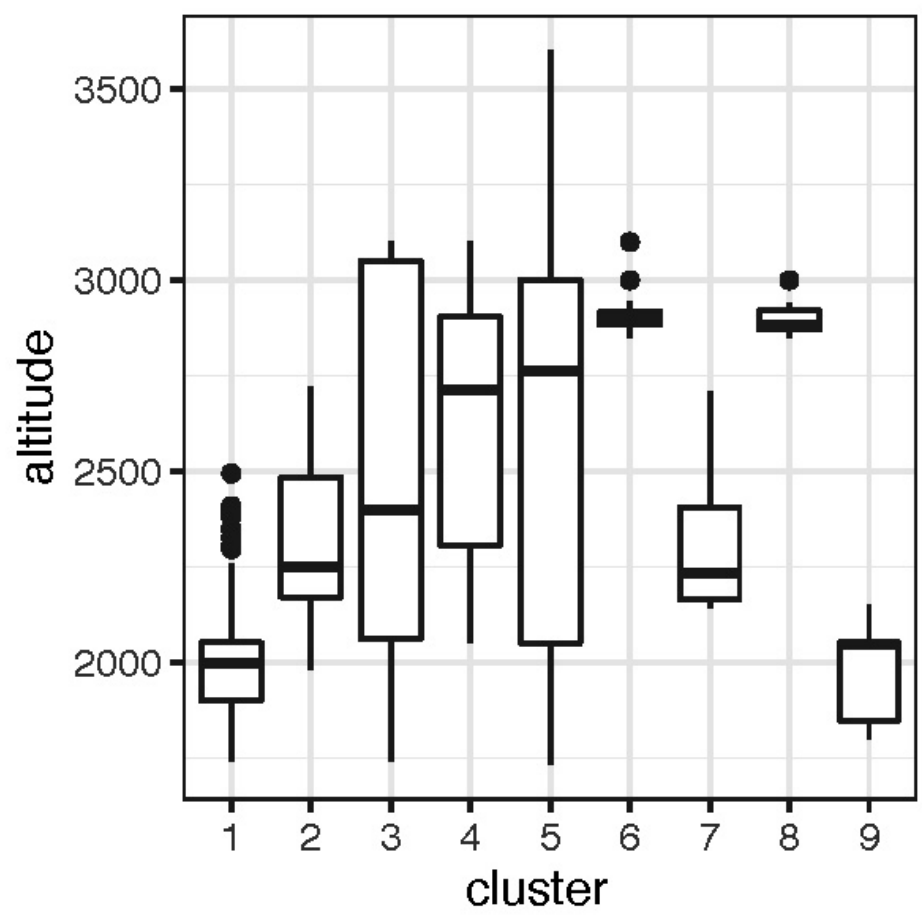

Figure 3b. Altitudinal distribution of the main phytosociological units.

(1: Bromopsio variegatae - Festucetea ovinea, 2: Nardo strictae - Caricetea pallescentis, 3: Festuco variae - Carietalia meinshausianae, 4: Carico tristis - Festueetalia supinae, 5: Sympoholomo graveolensis - Saxifragetea exaratae, 6: Sibbaldio semiglabratae - Gnaphalietea supinae, 7: Kobresio humilis - Kobresietalia capilliformis, 8: Alchemillo sericeae - Anthemetea sosnovskayae, 9: Rhinantho minoris - Trifolietalia pratensis) 

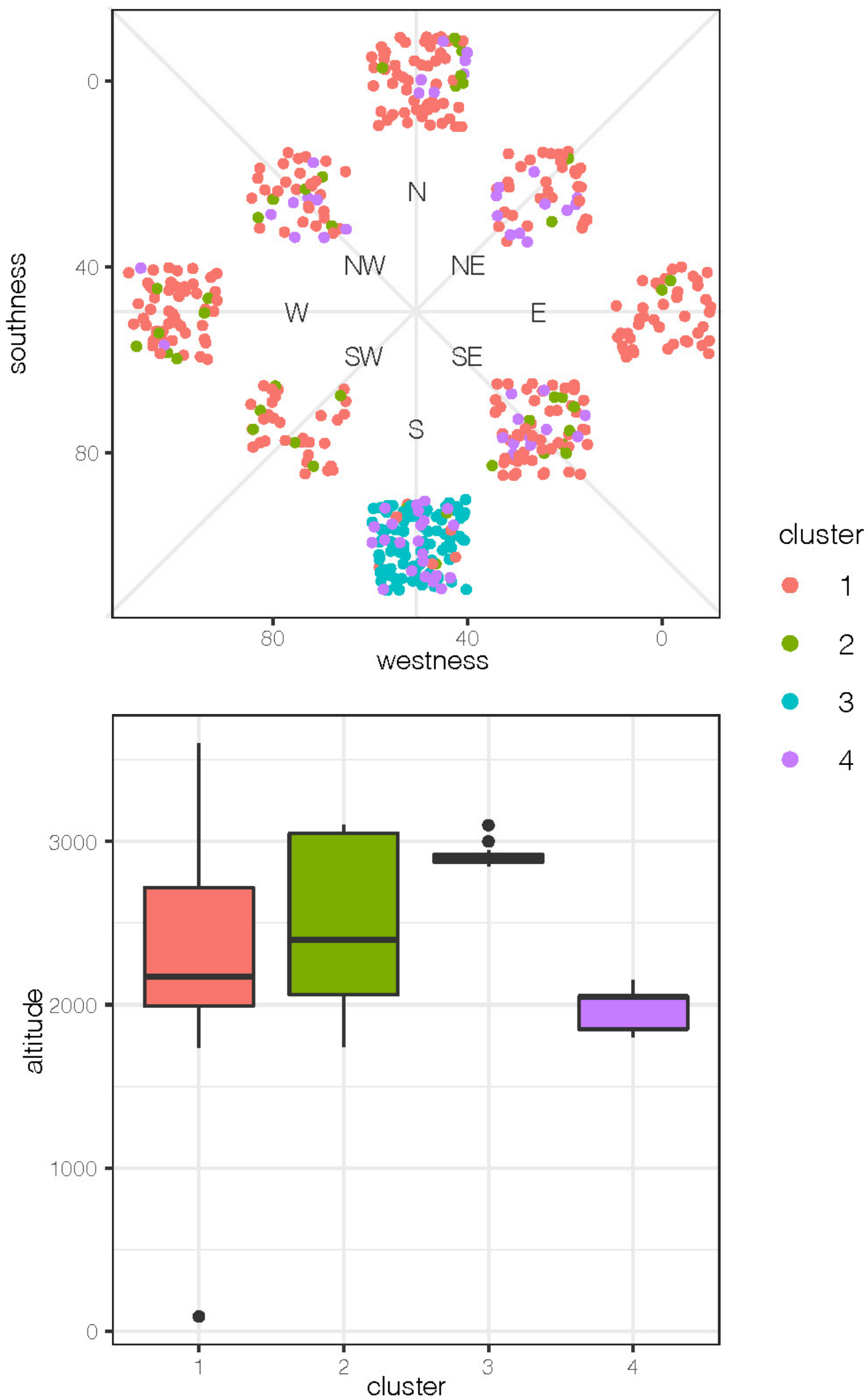

Figure 3c. Altitudinal distribution and exposition of main phytosociological units.

(1: Subalpine pastures Bromopsio variegatae - Festucetea ovinae, 2: Nutrient-rich alpine pastures: Festuco variae Carietalia meinshausianae, 3: Alpine pastures: Sympoholomo graveolensis - Saxifragetea exaratae, 4: Rhinantho minoris - Trifolietalia pratensis). 


\section{Community Assemblages}

The correlation between alpine pastures vegetation and environmental variables was indicated in the analysis obtained through PCA (Figures 4a,b). In constrained PCA ordination, the maximum explanatory variation was accounted on PC1 axis (15.9\%) and lower variation on PC2 axis (11.3\%). Maximum strength was recorded for the most important environmental gradient that is altitude and southern aspect among exposition variables. We observed from PCA ordination that environmental variables grouped the seven communities in three major groups (Figure 4): MC and PAA, ACD, FSF and TSA, and FBR and FNB. FBR and FNB showed positive relationship with SW, $N E, N, E$ aspect, but a negative with $S$ and altitude, once these two communities showed the lowest values of altitude (Figure 4). All other communities were positively related to altitude and $S$ aspect. Altitude $\left(R^{2}=0.5, p<0.001\right)$, SW $\left(R^{2}=0.02, p<0.007\right), N W\left(R^{2}=0.03, p<0.001\right), W\left(R^{2}=0.04, p<0.001\right), N\left(R^{2}=0.03, p<0.003\right)$ and $S\left(R^{2}=0.18\right.$, $p<0.001)$ aspect were the variables that significantly influenced communities' distribution in the ordination. The other aspects did not significantly influence (NE: $R^{2}=0.01, p=0.053, S E: R^{2}=0.01, p=0.100, W N: R^{2}=0.01, p=0.444, E$ : $\left.R^{2}=0.01, p=0.088\right)$. The FBR and FNB communities were found scattered due to the presence of associated species having vast ecological amplitude. In contrast with this, all the rest of five communities were found separately in clumps with clear differences based on their particular set of environmental variables (see Figure 2).

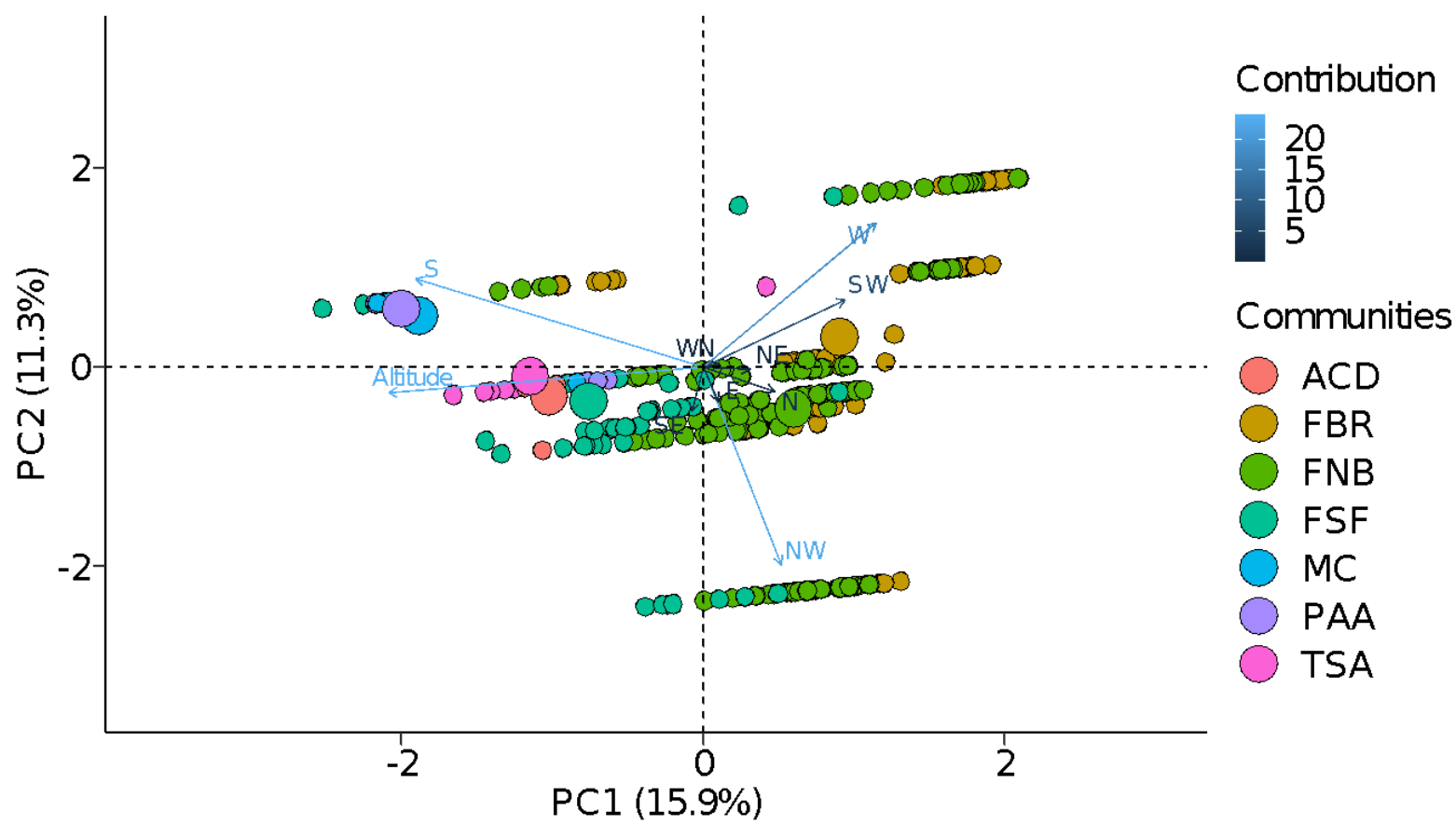

Figure 4a. Principal Component Analysis (PCA) illustrating the relationship of environmental variables with the seven different communities indicated in colored circles. Each circle with different colors indicates a separate community based on a $95 \%$ confidence level. Direction of the arrow illustrates the correlation of each environmental gradient, whereas length indicates the influence and strength of those gradients. Gradients on the same axis showed positive correlation while those on the opposite axes indicate negative correlation. Big circle demonstrates the centroid of each plant community. FBR: Festuca ovina, Bromopsis variegata, Ranunculus oreophilus, FNB: Festuca varia, Nardus stricta, Bromopsis variegata, FSF: Festuca varia, Sibbaldia semiglabra, Festuca supina, PAA: Poa alpina, Anthemis sosnovskyana, Aetheopappus caucasicus, TSA: Tripleurospermum subnivale, Saxifraga moschata, Alopecurus dasyanthus, MC: Minuartia inamoena, Campanula saxifraga, ACD: Alopecurus glacialis, Cerastium cerastioides, Delphinium caucasicum 

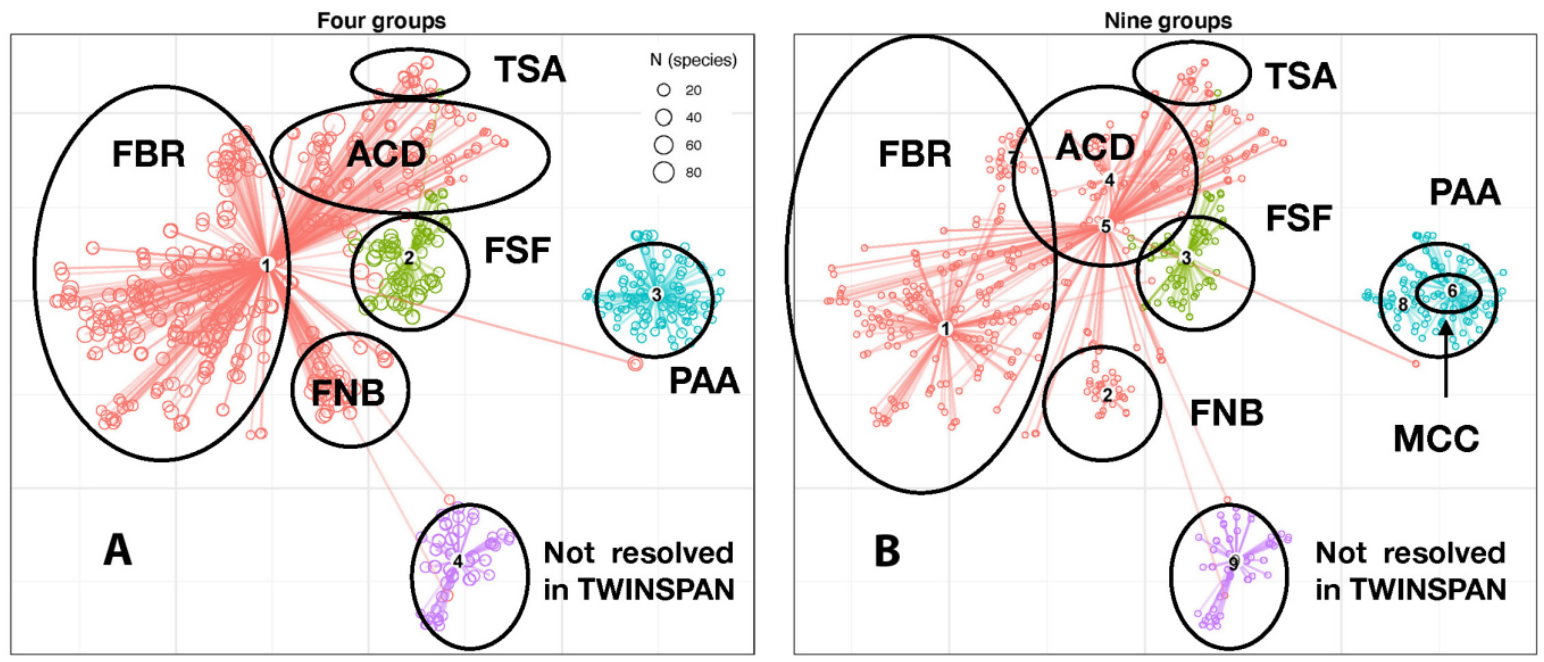

Figure 4b. Main Phytosociological units compared to TWINSPAM categorization

A: (1: Subalpine pastures Bromopsio variegatae - Festucetea ovinae, 2: Nutrient-rich alpine pastures: Festuco variae - Carietalia meinshausianae, 3: Alpine pastures: Sympoholomo graveolensis - Saxifragetea exaratae, 4: Rhinanthuo minoris - Trifolietalia pratensis.FBR: Festuc ovina, Bromopsis variegata, Ranunculus oreophilus, FNB: Festuca varia, Nardus stricta, Bromopsis variegata, FSF: Festuca varia, Sibbaldia semiglabra, Festuca supina, PAA: Poa alpina, Anthemis sosnovskyana, Aetheopappus caucasicus, TSA: Tripleurospermum subnivale, Saxifraga moschata, Alopecurus dasyanthus, MC: Minuartia inamoena, Campanula saxifraga, ACD: Alopecurus glacialis, Cerastium cerastioides, Delphinium caucasicum

B: (1: Bromopsio variegatae - Festucetea ovinea, 2: Nardo strictae - Caricetea pallescentis, 3: Festuco variae Carietalia meinshausianae, 4: Carico tristis - Festucetalia supinae, 5: Sympoholomo graveolensis - Saxifragetea exaratae, 6: Sibbaldio semiglabratae - Gnaphalietea supinae, 7: Kobresio humilis - Kobresietalia capilliformis, 8: Alchemillo sericeae - Anthemetea sosnovskayae, 9: Rhinantos minoris - Trifolietalia pratensis), FBR: Festuca ovina, Bromopsis variegata, Ranunculus oreophilus, FNB: Festuca varia, Nardus stricta, Bromopsis variegata, FSF: Festuca varia, Sibbaldia semiglabra, Festuca supina, PAA: Poa alpina, Anthemis sosnovskyana, Aetheopappus caucasicus, TSA: Tripleurospermum subnivale, Saxifraga moschata, Alopecurus dasyanthus, MC: Minuartia inamoena, Campanula saxifraga, ACD: Alopecurus glaciali,s Cerastium cerastioides, Delphinium caucasicum

\section{Species Composition}

Plant species composition significantly varied among communities (Table 2). All communities showed a significant difference in species composition between each other (Table 3.) Based on the overall Bray-Curtis dissimilarity of species between communities (Table 4), we identified eighteen plant species that most contributed to the overall dissimilarity, namely Achillea millefolium, Alopecurus dasyanthus, Alopecurus glacialis, Bromopsis variegata, Campanula saxifraga, Carex tristes, Cerastium cerastoides, Delphinium caucasicum, Festuca ovina, Festuca supina, Festuca varia, Kobresia capillifolia, Kobresia capillifolia, Kobresia capilliformis, Minuartia inamoena, Nardus stricta, Poa alpina, Ranunculus oreophilus, Saxifraga moschata, Sibbaldia semiglabra, and Tripleurospermum subnivale.

Table 2. PERMANOVA results comparing species composition between the four communities found in Moist temperate forest. This analysis was made with Euclidean distance and 999 permutations. Pairwise comparisons between communities are depicted in Table 2.

\begin{tabular}{lllllll}
\hline & Df & Sums of Sqs & Mean Sqs & $\mathrm{F}$ & $\mathrm{R}^{2}$ & $\operatorname{Pr}(>\mathrm{F})$ \\
\hline Communities & 6 & 104000 & 17333.3 & 8.77 & 0.079 & 0.0001 \\
Residuals & 612 & 1209567 & 1976.4 & & 0.920 & \\
Total & 618 & 1313567 & & & 1 & \\
\hline
\end{tabular}


Table 3. Pairwise comparisons with FDR p-value adjustment method of species composition between communities after PERMANOVA analysis (Table 1). Analyses were based on Euclidean distance and 999 permutations.

\begin{tabular}{lllllll}
\hline & ACD & FBR & FNB & FSF & MC & PAA \\
\hline FBR & 0.0016 & - & - & - & - & - \\
FNB & 0.0098 & 0.0016 & - & - & - & - \\
FSF & 0.0105 & 0.0016 & 0.0016 & - & - & - \\
MC & 0.0045 & 0.5020 & 0.4998 & 0.3990 & - & - \\
PAA & 0.0016 & 0.0016 & 0.0016 & 0.0016 & 0.1190 & - \\
TSA & 0.0016 & 0.0016 & 0.0016 & 0.0016 & 0.1001 & 0.0016 \\
\hline
\end{tabular}

Table 4. Contrast table of the three species that most contributed to the overall Bray-Curtis dissimilarity of species composition between the seven communities identified in our study. Av. dis. Average dissimilarity, SD Standard deviation, Av Com1 Average Community 1, Av Com2 Average community 2, Cum. Cumulative, Cont. Contribution.

\begin{tabular}{|c|c|c|c|c|c|c|c|c|c|}
\hline Communities & Plant species & Av dis & SD & Ratio & Av Com1 & Av Com2 & Cum & $\begin{array}{l}\text { Cum } \\
\%\end{array}$ & $\begin{array}{l}\text { Cont } \\
\%\end{array}$ \\
\hline \multirow[t]{3}{*}{ ACD-FBR } & Alo.gla & 0.3 & 0.2 & 1.3 & 0 & 28 & 0.3 & 27 & 27 \\
\hline & Cer.cer & 0.1 & 0.2 & 0.4 & 0 & 10.8 & 0.4 & 35.3 & 8.3 \\
\hline & Fes.ovi & 0.1 & 0.1 & 0.6 & 8.6 & 0 & 0.4 & 40.7 & 5.4 \\
\hline \multirow[t]{3}{*}{ ACD-FNB } & Alo.gla & 0.2 & 0.1 & 1.4 & 0 & 28 & 0.2 & 16.2 & 16.2 \\
\hline & Fes.var & 0.1 & 0.1 & 0.5 & 10.3 & 0 & 0.2 & 22 & 5.7 \\
\hline & Cer.cer & 0.1 & 0.1 & 0.4 & 0 & 10.8 & 0.3 & 27.6 & 5.6 \\
\hline \multirow[t]{3}{*}{ ACD-FSF } & Alo.gla & 0.3 & 0.2 & 1.3 & 0.8 & 28 & 0.3 & 26 & 26 \\
\hline & Cer.cer & 0.1 & 0.2 & 0.4 & 0.1 & 10.8 & 0.3 & 34.2 & 8.3 \\
\hline & Fes.var & 0.1 & 0.1 & 0.6 & 9.8 & 0 & 0.4 & 42.2 & 8 \\
\hline \multirow[t]{3}{*}{$A C D-M C$} & Alo.gla & 0.6 & 0.3 & 2 & 28 & 0 & 0.6 & 59.4 & 59.4 \\
\hline & Cer.cer & 0.1 & 0.3 & 0.4 & 10.8 & 0 & 0.7 & 74.3 & 15 \\
\hline & Del.cau & 0.1 & 0.2 & 0.5 & 3.5 & 0 & 0.8 & 81.6 & 7.2 \\
\hline \multirow[t]{3}{*}{ ACD-PAA } & Alo.gla & 0.5 & 0.3 & 2 & 0 & 28 & 0.5 & 54.8 & 54.8 \\
\hline & Cer.cer & 0.1 & 0.3 & 0.4 & 0 & 10.8 & 0.7 & 69.1 & 14.3 \\
\hline & Del.cau & 0.1 & 0.1 & 0.5 & 0 & 3.5 & 0.8 & 75.9 & 6.8 \\
\hline \multirow[t]{3}{*}{ ACD-TSA } & Alo.gla & 0.3 & 0.2 & 1.5 & 0.3 & 28 & 0.3 & 29.3 & 29.3 \\
\hline & Tri.sub & 0.1 & 0.2 & 0.8 & 14.1 & 0 & 0.4 & 43.4 & 14.1 \\
\hline & Cer.cer & 0.1 & 0.2 & 0.4 & 0.3 & 10.8 & 0.5 & 52.8 & 9.4 \\
\hline \multirow[t]{3}{*}{ FBR-FNB } & Fes.var & 0.1 & 0.1 & 0.5 & 1.1 & 10.3 & 0.1 & 5.9 & 5.9 \\
\hline & Bro.var & 0 & 0.1 & 0.8 & 8.2 & 7.6 & 0.1 & 11.1 & 5.2 \\
\hline & Fes.ovi & 0 & 0.1 & 0.7 & 8.6 & 4.6 & 0.2 & 16 & 4.9 \\
\hline \multirow[t]{3}{*}{ FBR-FSF } & Fes.var & 0.1 & 0.1 & 0.6 & 1.1 & 9.8 & 0.1 & 7.5 & 7.5 \\
\hline & Sib.sem & 0 & 0.1 & 0.5 & 0 & 8.7 & 0.1 & 12.3 & 4.8 \\
\hline & Fes.ovi & 0 & 0.1 & 0.6 & 8.6 & 0 & 0.2 & 17.1 & 4.8 \\
\hline \multirow[t]{3}{*}{ FBR-MC } & Fes.ovi & 0.1 & 0.1 & 0.7 & 8.6 & 0 & 0.1 & 8.4 & 8.4 \\
\hline & Bro.var & 0.1 & 0.1 & 0.8 & 8.2 & 0 & 0.2 & 16.1 & 7.8 \\
\hline & Ran.ore & 0 & 0.1 & 0.6 & 5.1 & 0 & 0.2 & 20.6 & 4.4 \\
\hline \multirow[t]{3}{*}{ FBR-PAA } & Fes.ovi & 0.1 & 0.1 & 0.7 & 8.6 & 0 & 0.1 & 8 & 8 \\
\hline & Bro.var & 0.1 & 0.1 & 0.8 & 8.2 & 0 & 0.2 & 15.4 & 7.4 \\
\hline & Ran.ore & 0 & 0.1 & 0.6 & 5.1 & 0 & 0.2 & 19.6 & 4.2 \\
\hline \multirow[t]{3}{*}{ FBR-TSA } & Tri.sub & 0.1 & 0.2 & 0.7 & 0 & 14.1 & 0.1 & 12.5 & 12.5 \\
\hline & Sax.mos & 0.1 & 0.1 & 0.6 & 0 & 8.1 & 0.2 & 19.1 & 6.5 \\
\hline & Alo.das & 0.1 & 0.1 & 0.5 & 0 & 7.7 & 0.3 & 25.2 & 6.1 \\
\hline \multirow[t]{3}{*}{ FNB-FSF } & Fes.var & 0.1 & 0.1 & 0.7 & 10.3 & 9.8 & 0.1 & 8.2 & 8.2 \\
\hline & Nar.str & 0 & 0.1 & 0.4 & 8 & 0.5 & 0.1 & 12.6 & 4.3 \\
\hline & Car.tri & 0 & 0.1 & 0.6 & 5.8 & 3.9 & 0.2 & 16.7 & 4.1 \\
\hline \multirow[t]{3}{*}{ FNB-MC } & Fes.var & 0.1 & 0.2 & 0.5 & 10.3 & 0 & 0.1 & 7.9 & 7.9 \\
\hline & Nar.str & 0.1 & 0.1 & 0.4 & 8 & 0 & 0.1 & 14 & 6.1 \\
\hline & Kob.cap & 0 & 0.1 & 0.4 & 6.8 & 0 & 0.2 & 19 & 5 \\
\hline \multirow[t]{3}{*}{ FNB-PAA } & Fes.var & 0.1 & 0.1 & 0.5 & 10.3 & 0.1 & 0.1 & 59.4 & 59.4 \\
\hline & Nar.str & 0.1 & 0.1 & 0.4 & 8 & 0 & 0.1 & 74.3 & 15 \\
\hline & Kob.cap & 0 & 0.1 & 0.4 & 6.8 & 0 & 0.2 & 81.6 & 7.2 \\
\hline \multirow[t]{2}{*}{ FNB-TSA } & Tri.sub & 0.1 & 0.1 & 0.8 & 0 & 14.1 & 0.1 & 7.8 & 7.8 \\
\hline & Fes.var & 0.1 & 0.1 & 0.5 & 10.3 & 0.1 & 0.1 & 13.4 & 5.6 \\
\hline
\end{tabular}




\begin{tabular}{llllllllll}
\hline \multirow{2}{*}{ FSF-MC } & Nar.str & 0 & 0.1 & 0.4 & 8 & 0 & 0.2 & 17.8 & 4.4 \\
& Fes.var & 0.1 & 0.2 & 0.6 & 9.8 & 0 & 0.1 & 13.4 & 13.4 \\
& Sib.sem & 0.1 & 0.2 & 0.5 & 8.7 & 0 & 0.2 & 21.7 & 8.3 \\
FSF-PAA & Fes.sup & 0.1 & 0.1 & 0.5 & 5.9 & 0.2 & 0.3 & 28.4 & 6.8 \\
& Fes.var & 0.1 & 0.2 & 0.6 & 9.8 & 0.1 & 0.1 & 13.2 & 13.2 \\
& Sib.sem & 0.1 & 0.2 & 0.5 & 8.7 & 0.1 & 0.2 & 21.4 & 8.2 \\
FSF-TSA & Fes.sup & 0.1 & 0.1 & 0.5 & 5.9 & 0.2 & 0.3 & 28.1 & 6.7 \\
& Tri.sub & 0.1 & 0.2 & 0.8 & 0.9 & 14.1 & 0.1 & 12.2 & 12.2 \\
& Fes.var & 0.1 & 0.1 & 0.6 & 9.8 & 0.1 & 0.2 & 20.1 & 7.8 \\
MC-PAA & Sax.mos & 0.1 & 0.1 & 0.6 & 0.3 & 8.1 & 0.3 & 26.5 & 6.4 \\
& Min.ina & 0.1 & 0.1 & 1.2 & 0.4 & 1 & 0.1 & 11.3 & 11.3 \\
& Poa.alp & 0.1 & 0.1 & 1.2 & 0.7 & 0.2 & 0.2 & 22.4 & 11.1 \\
MC-TSA & Cam.sax & 0.1 & 0.1 & 1.2 & 0.1 & 0.7 & 0.3 & 33.4 & 11 \\
& Tri.sub & 0.3 & 0.3 & 0.8 & 14.1 & 0 & 0.3 & 26.3 & 26.3 \\
& Sax.mos & 0.1 & 0.2 & 0.7 & 8.1 & 0 & 0.4 & 39.4 & 13 \\
PAA-TSA & Alo.das & 0.1 & 0.2 & 0.6 & 7.7 & 0 & 0.5 & 50.9 & 11.5 \\
& Tri.sub & 0.2 & 0.3 & 0.8 & 0 & 14.1 & 0.2 & 24.7 & 24.7 \\
& Sax.mos & 0.1 & 0.2 & 0.7 & 0 & 8.1 & 0.4 & 37 & 12.3 \\
& Alo.das & 0.1 & 0.2 & 0.6 & 0 & 7.7 & 0.5 & 48 & 11 \\
\hline
\end{tabular}

\section{Ordination of sampling plots}

Detrended Correspondence Analysis (DCA) ordination of all 619 plots of the study area were analyzed based on the plant species association. The floristic relationships among the major plant communities were visualized by means of DCA. The DCA resulted in correlation of 619 stands into the recognition of seven plant communities (Figure 5). Distribution of these recognized plant communities is dependent upon the presence of positively associated species within them, similar vegetation, and its linkage with the particular set of environmental variables and slope exposition. For instance, PAA community has been strongly influenced by the Southern aspect.

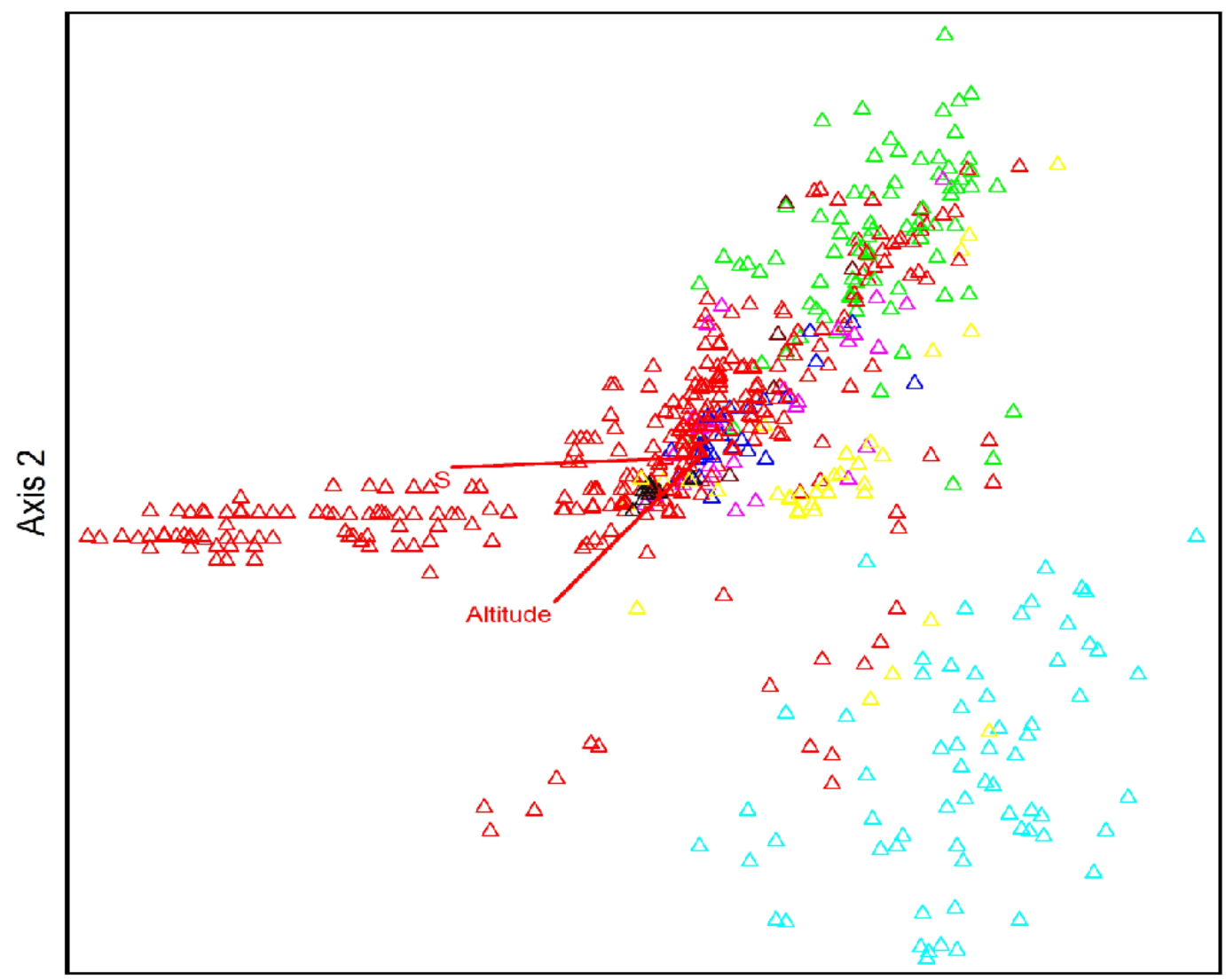

\begin{tabular}{|l|}
\hline Com \\
$\Delta 1$ \\
$\triangle 3$ \\
$\triangle 13$ \\
$\triangle 49$ \\
$\triangle 93$ \\
$\triangle 97$ \\
$\triangle 113$
\end{tabular}

Axis 1

Figure 5. DCA ordination of 619 plots further grouped into seven major plant communities. 


\section{Partial CCA}

Total inertia results of CCA were 40.825 , in which all variables were present in the final model and explained $4.2 \%$ of variation (sum of canonical eigenvalues was 1.753). The first two canonical axes explained $51.7 \%$ of variation. CCA model was significant $\left(x^{2}=1.753\right.$, pseudo- $F$ value $=2.727, p<0.001, d f=10$, permutations $\left.=999\right)$. Testing simple term effects for the 10 explanatory variables, we found that only six variables were significant $(p<0.05$, Table 5$)$. Performing the partial CCA for all the three possible classes (Table 6), the sum of the three classes explained $1 \%$ of the total variation (Figure 6.). Similar ordination techniques were used by other researchers including Haq et al. (2021a) from Pir Panchal mountain range of Himalayas and Rahman et al. (2022) from Pakistan Himalayas.

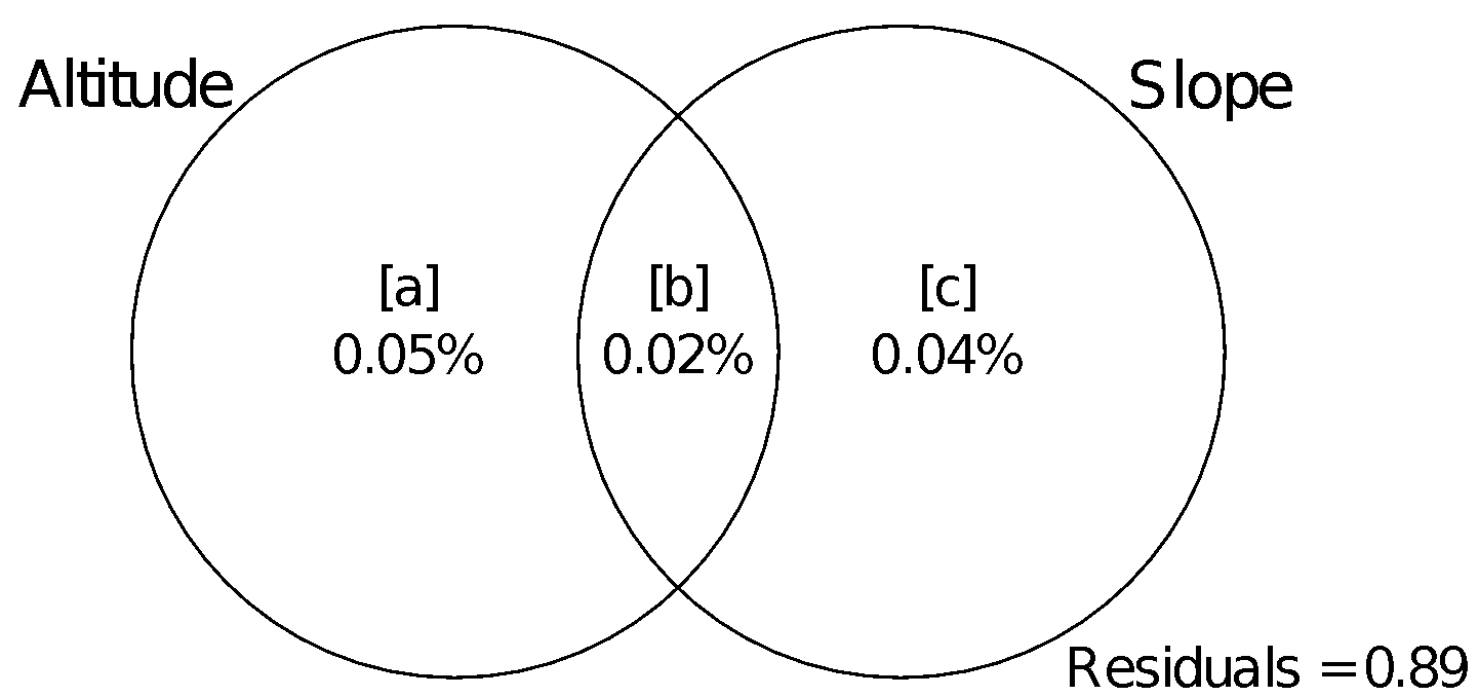

Figure 6. Venn diagram showing results of partial CCA and contribution (\%) of the two variable groups studied.

Table 5 The contribution and ranking of the studied variables in our partial CCA model. Significant variables are displayed in bold.

\begin{tabular}{lllll}
\hline & Df & ChiSquare & F & p-value \\
\hline Altitude & 1 & 0.632 & 98.396 & $\mathbf{0 . 0 0 1}$ \\
SW & 1 & 0.084 & 13.095 & 0.127 \\
NE & 1 & 0.079 & 12.311 & 0.163 \\
NW & 1 & 0.124 & 19.336 & $\mathbf{0 . 0 0 1}$ \\
SE & 1 & 0.082 & 12.816 & 0.167 \\
WN & 1 & 0.138 & 21.515 & 0.112 \\
W & 1 & 0.138 & 21.399 & $\mathbf{0 . 0 0 1}$ \\
N & 1 & 0.158 & 24.630 & $\mathbf{0 . 0 0 1}$ \\
E & 1 & 0.192 & 29.839 & $\mathbf{0 . 0 0 1}$ \\
S & 1 & 0.125 & 19.392 & $\mathbf{0 . 0 0 5}$ \\
\hline
\end{tabular}

Table 6. Results of variation partitioning (partial CCA) of four variable groups studied (see Figure VENN for individual fraction letter code).

\begin{tabular}{lllll}
\hline Individual Fraction & Adjusted $\mathbf{R}^{\mathbf{2}}$ & Variation explained (\%) & \% of all & Df \\
\hline [a] & 0.050 & 0.438 & 0.122 & 1 \\
[b] & 0.024 & 0.214 & 0.060 & 0 \\
[c] & 0.040 & 0.348 & 0.097 & 9 \\
Total explained & 11.396 & 1 & 0.279 & 10 \\
All variation & 40.825 & $/$ & 100 & \\
\hline
\end{tabular}




\section{Species richness and Diversity indices}

Species richness values differed significantly $\left(x^{2}=957.02, d f=6, p<0.001\right.$, Figure $\left.7 a\right)$ among seven plant communities, ranging from 06 to 350 plant species (Figure 7a). The highest number of plant species were recorded in the FNB community (350 species) at the lowest altitudinal range (1744m up to the middle range $2914 \mathrm{~m}$ ) followed by FBR (321 species) at the altitudinal range of $1735-2352 \mathrm{~m}$. Moreover, 173 plant species were recorded in the FSF community between $2075-3526 \mathrm{~m}$. TSA was the highest elevational range plant community recognized at $2800-$ $3600 \mathrm{~m}$ altitude with 36 associated species. Further, the lowest number of species was marked for MC community (six species) at the altitudinal range of alpine pastures $2870-3000 \mathrm{~m}$ ) in lowest number of plots (6 plots). FNB showed the highest average number of species per plot $28.2 \pm 0.8$, mean \pm SE) followed by FBR $21.5 \pm 0.6)$ and FSF (11.5 \pm 0.7$)$, which were significantly different from each other (Figure 7a). The other four communities showed an average of species per plot of 2.3-5.43 $\pm 0.2-0.9$.

We found significant difference of the four diversity indices, Species Richness Shannon $\left(x^{2}=476.22, d f=6, p<0.001\right.$, Figure $7 b)$, Simpson $\left(x^{2}=283.43, d f=6, p<0.001\right.$, Figure 7c), and Pielou's evenness $\left(x^{2}=440.03, d f=6, p<0.001\right.$, Figure $7 \mathrm{~d}$ ) between the seven plant communities. Again, the highest values of Shannon index were present in FBR $2.4 \pm 0.03$, mean \pm SE) and FNB 2.2 \pm 0.03 ) (Figure 7b). Intermediate values were observed in FSF $(1.6 \pm 0.06)$ and PAA $(1.5 \pm 0.04)$ (Figure 7c.And the lowest values were observed in TSA $(1.0 \pm 0.14)$, MC (0.8 \pm 0.08$)$ and ACD $(0.6 \pm 0.14 . F B R(0.84 \pm 0.01$, mean \pm SE), FNB $(0.79 \pm 0.01)$, and PAA $(0.75 \pm 0.01)$ showed the highest values of Simpson index (Figure 8c), followed respectively by FSF $(0.69 \pm 0.01), M C(0.55 \pm 0.03)$, TSA $(0.52 \pm 0.05)$, and ACD $(0.31 \pm 0.08$.Finally, MC $(1 \pm 0.0$, mean $\pm S E)$ and PAA $(0.98 \pm 0.01)$ showed the highest values of Pielou's evenness, followed respectively by FBR $(0.81 \pm 0.01)$, FSF $(0.71 \pm 0.01)$, TSA $(0.70 \pm 0.04)$, FNB $(0.68 \pm 0.1)$, and ACD $(0.48 \pm 0.08)$ (Figure $7 \mathrm{~d}$.).

a) Species richness

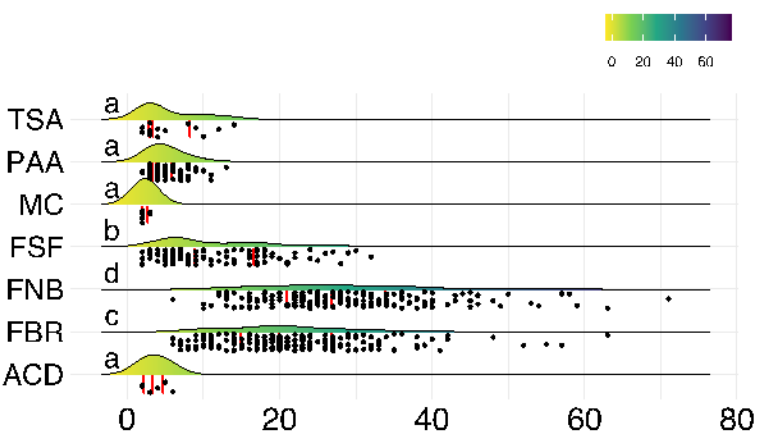

c) Simpson

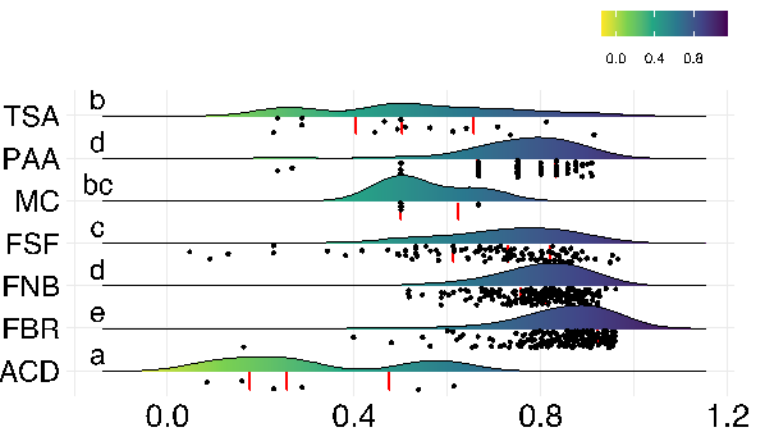

b) Shannon

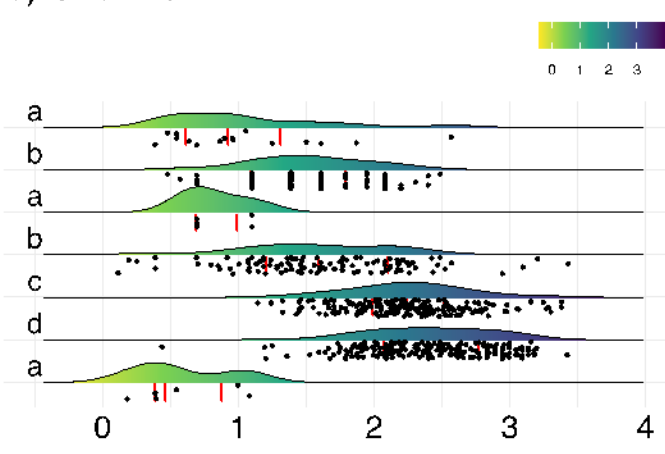

d) Pielou

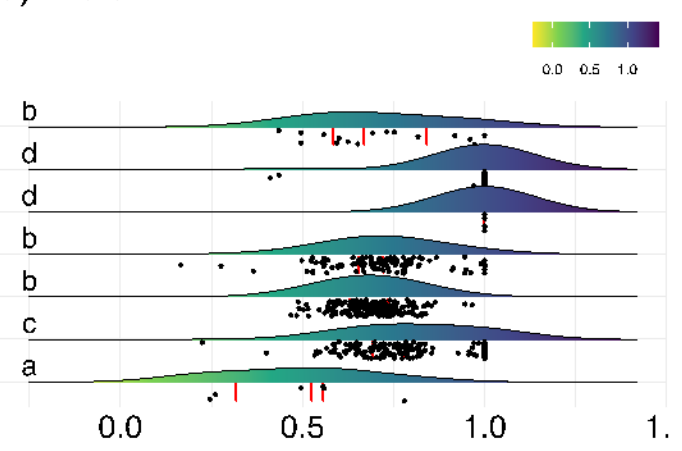

Figure 7. Variation of a) species richness, b) Shannon diversity, c) Simpson's diversity, and d) Pielou's evenness between the seven plant communities. Figures represent ridgeline plots with raw data (black dots below each density distribution) and first, second and third quartile (vertical red lines). Lowercase letters on the left differ from each other by estimated marginal means.

\section{Beta diversity (Bsim and 6sne)}

The total beta diversity ( $\beta$ sor) showed a value of $84.3 \%$ dissimilarity, of which spatial turnover ( $\beta$ sim) made up $48.8 \%$ and nestedness-resultant components ( $\beta$ sne) made up $35.4 \%$. In the $\beta$ sim cluster, we observed two major groups 
with $80 \%$ dissimilarity, one formed by TSA, FNB and ACD, and another formed by the other four communities (Figure 8). FBR was $66 \%$ dissimilar from PAA, MC and FSF cluster. MC and FSF were $100 \%$ similar to each other. TSA showed a dissimilarity of $38.8 \%$ with FNB and ACD. In the $\beta$ sne component, we found $93.3 \%$ dissimilarity between FSF, FNB and FBR cluster and TSA, PAA, MC and ACD cluster (Figure 8). FSF showed $24.4 \%$ dissimilarity with FNB and FBR. TSA and PAA showed $1 \%$ dissimilarity between each other, and $61 \%$ with the MC and ACD cluster (Figure 8). Similar classification was carried in High altitude vegetation in other parts of the world (Haq et al. 2021b; Yang et al., 2021).
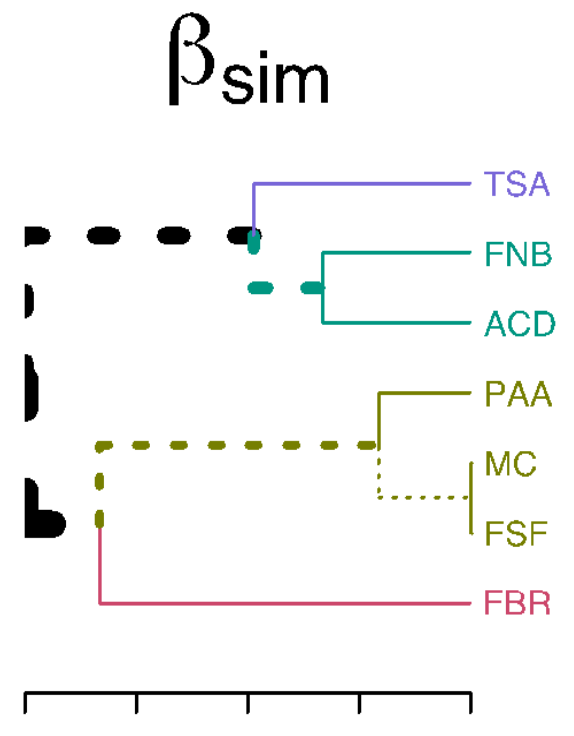
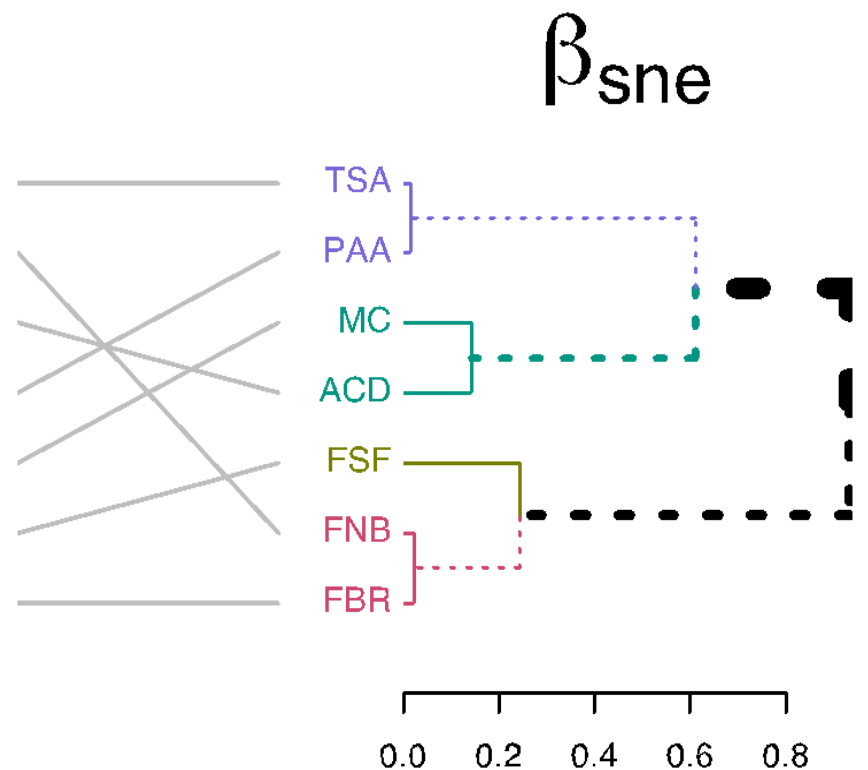

Figure 8. Dissimilarity cluster based on spatial turnover ( $\beta$ sim) and nestedness-resultant components ( $\beta$ sne) of beta diversity components of species dissimilarity between seven plant communities.

\section{Phytosociological classification}

A complete overview on phytosociological units, as well as all relevé data is given in Appendices 1 2-1 to 2-7 and 3 ) in the separate galleys.

Subalpine pastures Bromopsio variegatae - Festucetea ovinea (Class. nov.) (=FBR TWINSPAN) Type relevé:
N120
Main altitude: (1750)1994-2720(3600) m
Character species: Bromopsis variegata, Festuca ovina, Veronica gentianoides, Trifolium ambiguum, Leontodon
hispidus, Plantago atrata, Carum caucasicum, Ranunculus oreophilus, Poa alpina, Festuca supina, Alchemilla
sericata, Carex buschiorum
Subalpine meadows comprise all more or less mesic herbaceous communities which are either mown or grazed.
The Caucasian subalpine grassland is very diverse floristically, phytocoenotically and in terms of the spectrum of
life forms. Below we describe the main types of subalpine grassland, which are commonly used as hayfields and
some of them also as pastures. These communities occupy both dry and moist localities, they occur on skeletal
cobble soils. Main components of the above-mentioned coenoses are Trifolium ambiguum, Lotus caucasicus,
Alchemilla sericata, etc. The above communities are widespread in the Caucasian high-mountains and reach the
altitude of 2700 m. Tussocks are used as hay-meadows.
Bromopsis variegatae Festucetea ovinea communities occupy both dry and moist habitats. They occur on skeletal
eroded soils. The driest eroded slopes of the subalpine belt are covered by Bromopsietum ripariae communities.
They grow on thin, scree and skeletal soils. Among the most widespread communities in the relatively dry semi-
continental highlands of the Central Great Caucasus are Bromopsis variegata and Agrostis tenuis meadows. They
are distributed from the upper mountain to the alpine belt (up to 2700 m). In this belt, these communities occupy
flat and moderately sloping hillsides of almost all exposures. These meadows (sparse meadows corresponding to
'Magerwiesen') are distinguished by a large number of species. They are used both as hayfields and pastures.




\begin{abstract}
Astragalo capitosae - Thymetalia collinae (Ord. nov.) (=FBR TWINSPAN) Type relevé: N120
Main altitude: 1900-2053 m

Character species: Astragalus captiosus, Thymus collinus, Plantago atrata, Rhinanthus vernalis, Koeleria caucasica, Koeleria luerssenii, Galium verum, Agrostis tenuis, Pulsatilla violacea, Dianthus cretaceus, Pedicularis comosa, Trifolium alpestre, Bupleurum polyphyllum

In mostly stony places. Communities dominated by Astragalus captiosus are characteristic to the subalpine belt of the eastern part of the Central Great Caucasus. These communities are dominated by the endemic Astragalus captiosus and occupy steep rocks and screes. Companion species are: Carex buschiorum, Campanula bellidifolia, and Koeleria luerssenii. There is a variant on strongly eroded slopes, with species such as Festuca valesiaca, Bromopsis variegata, Lotus caucasicus, Alchemilla sericata, Pulsatilla violacea, Bupleurum polyphyllum, Trifolium alpestre, Koeleria luerssenii, Carex buschiorum. Festucetum valesiacae as well as Festucetum ovinae can also be frequently found in the alpine belt up to $2560 \mathrm{~m}$. For example, Kolteshi, steppes ascend often quite high, almost up to the alpine belt, though with elevation, these communities are depleted of xerophytes. Throughout the transect from 18002600 m, xerophilic vegetation is very sharply interrupted by mesophilic meadow communities.

Scabioso caucasicae - Agrostion planifoliae / Scabioso caucasicae - Agrostetum planifoliae typicum (All. / Ass. nov.) Type relevé: N120

Character species: Scabiosa caucasica, Agrostis planifolia, Phleum phleoides, Anemonastrum fasciculatum, Helictotrichon adzharicum, Hieracium umbellatum, Ranunculus acutilobus, Trifolium alpestre, Gentianella caucasea, Pedicularis comosa, Anthyllis variegata, Lotus caucasicus, Alchemilla debilis, Centaurea cheiranthifolia, Gentiana septemfida, Trifolium trichocephalum

Distributed almost throughout the Caucasus on moderately humid northern and north-western slopes, forest edges and openings. Characteristic companion species are: Trollius ranunculinus, Geranium ibericum, Betonica macrantha, Veratrum album, Polygonum carneum. Subalpine meadows with Veratrum album are abundant in the western and partly Central Great Caucasus. Veratrum album is a tall and poisonous pasture weed, usually rejected by animals, it is commonly associated with Trollius ranunculinus and Ranunculus caucasicus.
\end{abstract}

Agrostio planifoliae - Phleetum phleoideum (Ass. nov.) Type relevé: N308

Character species: Agrostis planifolia, Phleum phleoides, Ranunculus acutilobus

Sileno linearifoliae - Alchemillion sericeae / Sileno linearifoliae - Alchemilletum sericeae typicum (All. / Ass. nov.) Type relevé: F4

Character species: Silene linearifolia, Alchemilla sericata, Poa badensis, Scabiosa bipinnata, Asperula albovii, Pulsatilla violacea, Draba nemorosa, Campanula hohenackeri, Sedum acre, Sempervivum pumilum. Campanula bellidifolia

Typical for disturbed areas and are confined to slopes of southern exposure. Pulsatilla violacea is frequently associated with hemixerophitic grasses such as Festuca ovina, Koeleria luerssenii and with Carex buschiorum.

Campanulo hohenackeris - Pulsatilletum violaceae (Ass. nov.) Type relevé: F16

Character species: Campanula hohenackeri, Pulsatilla violacea, Trifolium alpestre, Campanula bellidifolia Differential species: Saxifraga juniperifolia, Sempervivum caucasicum, Festuca valesiaca, Thalictrum foetidum, Artemisia campestris, Sedum oppositifolium, Vicia grossheimii

Silieno linearifoliae - Scutellarietum oreophilae (Ass. nov.) Type relevé: N7003

Character species: Trifolium alpestre

Differential species: Silene linearifolia, Scutellaria oreophila, Rumex acetosella, Crepis sonchifolia

Rhinantho vernalis - Agrostion tenuis / Rhinantho vernalis - Agrostetum tenuis typicum (All. / Ass. nov.) Type relevé: N4

Character species: Rhinanthus vernalis, Agrostis tenuis, Alchemilla sericata, Cerastium arvense, Lotus caucasicus, Ranunculus caucasicus, Trifolium fontanum, Centaurea cheiranthifolia, Euphrasia hirtella, Pedicularis comosa, Myosotis arvensis, Bromopsis variegata, Alchemilla rigida 
Alchemilo rigidae - Polygonetum alpinae (Ass. nov.) Type relevé: N37

Character species: Alchemilla rigida, Polygonum alpinum, Ranunculus acutilobus

Differential species: Pedicularis comosa, Silene wallichiana, Pastinaca armena, Campanula oblongifolia, Tragopogon reticulatus

Potentillo erectea - Campanuletum bellififoliae (Ass. nov.) Type relevé: N39

Character species: Pulsatilla violacea, Campanula bellidifolia

\section{Nardo strictae - Caricetea pallescentis (Ord. nov.) (=FNB TWINSPAN) Type relevé: N52} Main altitude: $2172-2484 \mathrm{~m}$

Character species: Nardus stricta, Carex pallescens, Prunella vulgaris, Trifolium trichocephalum, Anthoxanthum alpinum, Potentilla erecta, Leontodon danubialis, Cerastium purpurascens, Cruciata laevipes, Daphne glomerata, Chaerophyllum roseum

Nardus-Caricetea communities are widespread throughout the Caucasus, mainly on gentle slopes of almost all exposures. Due to overgrazing, the secondary forms of Nardeta communities are predominant. The primary Nardeta communities are confined to the slopes around glaciers, and in the upperpart of steep gullies. Mixed communities of Nardeto-Festucetum variae can be found in the central and eastern parts of the Great Caucasus. Under the influence of pasturing hygrophytic Nardus communities have developed on wetlands in depressions of alpine belt. Nardetum strictae companion species are: Avenella flexuosa, Alchemilla caucasica, Sibbaldia semiglabra, Taraxacum stevenii, Cerastium purpurascens, Geranium gymnocaulon, Pedicularis crassirostris, Phleum alpinum

Potentillo erectae - Leontodontion danubialis / Potentillo erectae - Leontodonetum danubialis typicum (All. / Ass. nov.) Type relevé: N52

Character species: Plantago lanceolata, Cynosurus cristatus, Vaccinium myrtillus, Polygala alpicola, Ajuga orientalis, Phleum alpinum.

Differential species: Campanula trautvetteri, Luzula pseudosudetica, Viola somchetica

Carico tristis - Festucetalia supinae (Ord. nov.) Type relevé:

Main altitude: $2300-2900 \mathrm{~m}$

Character species: Carex tristis, Festuca supina, Alchemilla caucasica, Polygonum viviparum, Dryas caucasica, Thalictrum alpinum, Potentilla gelida, Astragalus supinus

The areas occupied by these communities are not large. Habitat: Stony, calcareous soils on steep 20-50 ) slopes with North and North-West aspects. Relatively wide in the Truso gorge and on the Mt. Kuro slopes, between 2000 and $2600 \mathrm{~m}$. Among the sedge dominated grasslands the Carex tristis Festucetalia supinae occupies the upper levels of the closed alpine vegetation and is widespread in the Caucasus. Usually, they inhibit prominent slopes exposed to winter winds. Carex tristis participates in the formation of the sedge-fescue association (Festuca supina Carex tristis). The most typical communities are: Cariceto-Alchemilleta (Alchemilla caucasica) and CaricetoKobresieta (Kobresia macrolepis.). Companion species are: Carex tristis, Festuca supina, Thalictrum alpinum, Eritrichium caucasicum, Polygonum viviparum, Antennaria caucasica, Anemone speciosa.

Minuartion imbricatae / Minuartietum imbricatae typicum (All. / Ass. nov.) Type relevé: F12

Character species: Minuartia imbricata, Potentilla gelida, Erigeron alpinus, Anemone speciosa, Astragalus alpinus, Empetrum nigrum, Antennaria caucasica, Luzula spicata, Androsace villosa

Festucetum supinae communities chiefly occupy the highest elevations of the belt 2700-2900 m), steep or slightly inclined slopes. Companion species are: Kobresia macrolepis, K. persica, Astragalus supinus, Polygonum viviparum, Thalictrum alpinum, Alchemilla caucasica, Anemone speciosa, Carex tristis, Lomatogonium carinthiacum. Relatively dry variant of the meadows also occurs with participation of steppe vegetation: Pulsatilla violacea, Koeleria luerssenii.

Euphrasio petiolaris- Campanulaetum ciliatae (Ass. nov.) Type relevé: F12

Character species: Antennaria caucasica, Campanula biebersteiniana

Differential species: Euphrasia petiolaris, Campanula ciliata, Kobresia capillifolia, Lomatogonium carinthiacum 
Primulo amoenea - Desachampsietum caespitosae (Ass. nov.) Type relevé: N162

Character species: Anemone speciosa, Festuca supina, Veronica gentianoides

Differential species: Primula amoena, Deschampsia cespitosa, Matricaria caucasica, Leontodon caucasicus, Eritrichium caucasicum, Festuca varia subsp. woronowii communities in the alpine belt occupy rather steep slopes of southern exposure and occur within the entire mountainous part of the Caucasus. Most frequently they occur in the eastern part of the Great Caucasus. Companion species are: Festuca supina, Carex tristis, Kobresia schoenoides, Astragalus supinus, Luzula spicata, Polygonum viviparum, Alchemilla sericea, Fritillaria lutea.

\section{Sibbaldio semiglabratae - Gnaphalietea supinae (Ord. nov.) Type relevé: N178} Main altitide: 2050-3000 m

Character species: Sibbaldia semiglabra, Gnaphalium supinum, Geranium gymnocaulon, Saxifraga moschata, Taraxacum stevenii, Colpodium versicolor, Hordeum violaceum, Campanula biebersteiniana

Sibbaldia semiglabratae Gnaphalietea supinae communities occupy gentle slopes and alluvial cones in the lower part of the subalpine belt (1800-2200 m). Colpodietum versicolor communities occupy small areas between the skeletal substrates and moraines. They are widespread in the upper alpine and subnival belts. Companion species are: Alopecurus glacialis, Veronica telephïfolia, Potentilla crantzii, Taraxacum stevenii, Lamium tomentosum, Nepeta supina. These species are both typical alpine and subalpine belts.

Seselio transcaucasicae - Trifolion arureum / Seselio transcaucasicuae - Trifolietum arureum typicum (All. / Ass. nov.) Type relevé: $\mathbf{N} 268$

Character species: Seseli transcaucasicum, Trifolium aureum, Sibbaldia parviflora, Alchemilla retinervis, Saxifraga moschata, Avenella flexuosa, Ranunculus grandiflorus, Trifolium fontanum, Cerastium polymorphum, Carex medwedewii

Saxifrago sibiricae - Cerastietum cerastioidis (Ass. nov.) (=ACD TWINSPAN) Type relevé:

Character species: Saxifraga moschata Geranium sylvaticum, Lamium tomentosum Blysmus compressus, Cerastium polymorphum, Colpodium versicolor

Differential species: Saxifraga sibirica, Cerastium cerastoides, Delphinium caucasicum, Gladiolus tenuis

Snowbed communities are composed of plant species that require or tolerate longterm snow cover (7-9 months, and sometimes throughout the year.Snowbed vegetation of the central European Alps belongs to the Saliceta herbacea Arctic Alpine community on siliceous substrate (Englisch 1993.Some of these snowbed species are found in the Caucasus as well as in the Alps: Cerastium cerastoides, Plantago atrata subsp. saxatilis, Sagina saginoides, Gnaphalium supinum, and Sibbaldia procumbens, which is a relative of $S$. parviflora. The floristic richness of these communities is low. Soil is permanently moist and supports the development of bryophytes. Humus content is low and nutrient availability is believed to be low. However, the snow supplies these communities with organic dust, which is partly mineralized by bacteria and fungi. In contrast to the Alps and the Scandinavian mountains, species such as Salix herbacea do not occur in alpine areas of the Caucasus (Ellenberg 1996.Similarly, another typical snowbed species of the Alps, Soldanella pusilla, is not present. Two phanerogam species in the alpine zone of the Central Great Caucasus that are dependent on reliable snow cover in winter, are Daphne glomerata and Rhododendron caucasicum, but these species clearly do not belong to snowbed communities. In Europe, typical snowbed communities cover large areas (hundreds of square meters each). By floristic composition snowbed communities of the Central Great Caucasus are apparently distinct from snowbed communities of the Anatoliam mountains, actually none of the species are identical (Kürschner et al. 1998).

In the Caucasus, these snowbed communities are composed of low stature geophytes and caespitose forb species, such as Galanthus platyphylllus, G. caucasicus, Gagea sulphurea, G. glacialis, G. anisanthos, Merendera raddeana, Ornithogalum schmalhausenii, Puschkinia scilloides, Primula algida, P. ruprechtii, Campanula biebersteiniana, Minuartia aizoides, Taraxacum stevenii, Cerastim cerastoides, Gnaphalium supinum, and Sibbaldia parviflora, and they include small grasses, such as Poa alpina and Phleum alpinum, as well as Carex medwedewii, Alchemilla rigida.

(a) Isolated plants are present in all biotopes of the subnival belt with the exception of snowbeds. For example, on unstable screes: Cerastium kasbek, Delphinium caucasicum, on slightly more stable substrate: Veronica minuta, V. telephïfolia, Scrophularia minima, Lamium tomentosum, on stable substrate: Aetheopappus caucasicus, Symphyoloma graveolens, Jurinella subacaulis, Minuartia inamoena, on rock Primula bayernii, Draba bryoides, Saxifraga exarata subsp. moschata. All these plants are chionophobes or hemichionophobes and have different 
habit: Prostrate plants such as Veronica minuta and V. telephïfolia, Acaulescent rosette plants such as Scrophularia minima, Symphyoloma graveolens, Aetheopappus caucasicus, Cushion plants such as Minuartia inamoena, M. trautvetteriana, Saxifraga exaratas subsp. moschata and Draba bryoides. Almost all above-mentioned species are typical representatives of the subnival belt and are well adapted to these harsh environmental conditions.

Pastinaco armenae - Agrostietum planifoliae (Ass. nov.) Type relevé: N1023

Character species: Pastinaca armena, Agrostis planifolia

Differential species: Poa nemoralis, Anthriscus nemorosa, Phleum montanum, Bromopsis riparia

Sibbaldio parviflorae - Alchemilletum retinervis (Ass. nov.) Type relevé: N167

Character species: Sibbaldia parviflora, Alchemilla retinervis, Carex medwedewii, Phleum alpinum, Anthoxanthum alpinum, Leontodon caucasicus, Avenella flexuosa, Daphne glomerata, Taraxacum stevenii

Differential species: Carex caryophyllea, Hieracium laevigatum

Sibbaldio semigrabrae - Geranion gymnocaulae / Sibbaldio semigrabrae - Geranietum gymnocaulae typicum (All. / Ass. nov.) (=TSA TWINSPAN) Type relevé: N1035

Character species: Sibbaldia semiglabra, Geranium gymnocaulon, Gnaphalium supinum, Colpodium versicolor, Taraxacum stevenii, Phleum alpinum, Potentilla gelida, Festuca supina, Corydalis alpestris

Differential species: Pedicularis crassirostris, Minuartia aizoides, Alopecurus glacialis, Tripleurospermum subnivale, Luzula multiflora

Alchemilla glabricaulis

Sibbaldio semigrabrae - Geranion gymnocaulae communities are distributed in the Western and Central Great Caucasus. According to Dolukhanov (1946) as well as Onipchenko 2004), Geranium communities extend from the lower part of the alpine belt to the upper limits of dense vegetation, these meadows merge into the subnival belt. They occur on mountain-meadow skeletal soils. Sibbaldia parviflora and S. semiglabra are important taxa in both alpine and nival herbfields, yet their taxonomic status is unclear. The majority of Caucasian botanists recognize these two species as independent, while others only rank Sibbaldia parviflora as a recognised species, with Sibbaldia semiglabra considered to be a synonym (Kolakovsky 1985, Zernov 2006, Güner 2012.Some botanists (e.g., Onipchenko 2004) deny both Caucasian species and consider them resembling the European species Sibbaldia procumbens. Kamelin 2001) recognized only S. parviflora but considers it to be very close to S. procumbens. Carpetforming alpine herbfields, addressed as 'Dicotylen-Teppiche' by Ellenberg (1996), are a very diverse group of communities spread over the upper part of the alpine belt. Caucasian botanists call it 'the upper alpine sub-belt' (Dolukhanov et al. 1946.Along with snowbeds the carpet-like herbfields are distributed on volcanic scree, rocks and between moraines and large stones. These small herb-fields occupy sheltered gaps among large boulders and gullies, often characterized by long-term snow cover. A short growing season is typical for all these herbfields. The plant canopy commonly does not exceed a height of $3-4 \mathrm{~cm}$ and $85 \%$ of aboveground phytomass is accumulated in the 0-1 cm layer, thus, forming dense swards. Roots in the upper 5-6 cm layer form a thick felt. A second, lower layer consists of sparse roots down to $20-25 \mathrm{~cm}$. Some species have large and brightly coloured inflorescences while others are inconspicuous. The term 'Nanoherbeta' has been attributed to such herbfields. Besides these primary carpet-like alpine herbfields, vast alpine areas are occupied by secondary herbfield carpets with a dominance of Alchemilla spp. as a result of high manure input and cattle resting. Another species-poor variant of secondary and alpine herbfields follows from overgrazing and trampling on dry soils with a dominance of Sibbaldia semiglabra and S. parviflora. On moist sites, carpet-like herbfields with Carum caucasicum develop with companion species such as Taraxacum stevenii, Campanula biebersteiniana, Plantago atrata subsp. saxatilis and Minuartia aizoides. Carpet-like communities are also developed around late laying patches of snow and at the edges of the glaciers and they include Ranunculus oreophilus var. pumilus, $R$. baidarae, Primula algida, Gentiana pyrenaica, $G$. angulosa, G. nivalis, Minuartia aizoides, and Cerastium cerastoides. In wet conditions, on the banks of meltwater streams Pedicularis crassirostris, P. nordmanniana, Primula auriculata, and Poa alpina become prominent. Alpine carpet-like communities are characterized by the same species in the entire Euxine province (Anatolia, the Great and Lesser Caucasus mountains) and are forming plant communities made up of Sibbaldia parviflora-Campanula biebersteiniana or Alchemilla retinervisSibbaldia parviflora (Fedorov 1942, Mikeladze 1960, Narinyan 1959, Kolakovsky 1961, Onipchenko 2004, Zernov 2006, Nakhutsrishvili 2013). 
Colpodium versicolor Corydalis alpestris variety Type relevé: N3007

Character species: Colpodium versicolor, Corydalis alpestris

Kobresio schoenoideea - Minuartion oreinae / Kobresio schoenoideae - Minuartietum oreinae typicum (All. / Ass. nov.) Type relevé: $\mathbf{N} 3017$

Character species: Kobresia schoenoides, Minuartia oreina, Alchemilla sericata, Salix caprea, Anthemis marschalliana, Minuartia aizoides, Briza marcowiczii, Campanula tridentata, Campanula petrophila, Saxifraga kolenatiana, Trollius ranunculinus, Myosotis alpestris, Androsace villosa, Saxifraga flagellaris, Helictotrichon asiaticum

Humid broad-leaved meadows with dominant Trollius ranunculinus are distributed in periodically wet places, they are found in forest openings as well as on slightly sloping hillsides and small depressions of both subalpine and alpine belts. The areas occupied by these meadows are not large. They occur as patches scattered over the other vegetation belts. Ranunculus caucasicus frequently occurs in these communities as a subdominant. Almost pure Ranunculus herb-fields were formed under the influence of overgrazing. Characteristic companion species are: Veratrum album, Dactylorhiza euxina, D. urvilleana, Poa alpina, Swertia iberica, Avenella flexuosa, Pedicularis crassirostris.

Alchemillo sericeae - Minuartio aizoides variety Type relevé: N1049

Character species: Alchemilla sericea, Minuartia aizoides, Minuartia oreina, Anthemis marschalliana Kobresia schoenoides communities occur mostly on stony localities and within variegated fescue grassland. Companion species are: Kobresia macrolepis, K. persica, Festuca supina, Astragalus alpinus, Anthemis marschalliana, Taraxacum stevenii, Anemone speciosa, Thalictrum alpinum, Alchemilla elisabethae, Polygonum viviparum. The Kobresietum is mainly spread along the wind direction on mountain ridges in both subalpine and alpine belts. Companion species are: Kobresia macrolepis, Alchemilla elisabethae, Carex tristis, Thalictrum alpinum, Polygonum viviparum, Alchemilla caucasica.

Kobresio humilis - Kobresietalia capilliformis (Ord. nov.) Type relevé: N195

Main altitude: $2165-2400 \mathrm{~m}$

Character species: Kobresia humilis, Kobresia capilliformis, Chamaesciadium acaule, Alchemilla elisabethae, Veronica gentianoides, Polygonum viviparum, Carex buschiorum, Trifolium ambiguum, Gentiana aquatica, Cirsium rhizocephalum, Primula algida, Minuartia circassica

Marginal ridges of the valley harbor specific vegetation reflecting extreme effects of wind, water erosion, frost action (soil polygons), and intensive grazing. The species composition of particular ridges is influenced by the steepness and size of the ridge as well as by contact communities.

Cirsio rhizocephalae - Primulion algidae / Cirsio rhizocephalae - Primuletum algidae typicum (All. / Ass. nov.) Type relevé: $\mathbf{N 1 9 5}$

Character species: Cirsium rhizocephalum, Primula algida, Trifolium ambiguum, Draba siliquosa, Taraxacum confusum, Carum caucasicum, Festuca supina, Scabiosa caucasica, Campanula collina, Anthyllis variegata

Gentiana aquatica variety Type relevé: N315

Character species: Gentiana aquatica

Typical spring ephemerals in uncut meadows are Primula amoena, P. cordifolia, Fritillaria lutea, F. latifolia F. caucasica, Gentiana aquatica, Iris reticulata.

Nutrient-rich alpine pastures: Festuco variae - Caricetalia meinshausianae (Ord. nov.) (=FSF TWINSPAN) Type relevé: N28

Main altitude: (1750)2060-3050(3150m)

Exposition: Never South, very rarely East or Northest

Character species: Festuca varia, Carex meinshauseniana, Betonica macrantha, Campanula collina, Polygonum carneum, Minuartia circassica, Helictotrichon adzharicum, Primula amoena, Inula orientalis, Gentiana septemfida Festuco variae - Caricetalia meinshausianae occupies vast slopes of the subalpine belt, forming the first stage of succession. Under the influence of intensive grazing, Calamagrostis arundinacea communities become replaced by those of variegated fescue, but after grazing was discontinued, these tussock grasslands became transformed. On wet slopes, Festuca communities are richer than on dry ones. Jaroshenko (1942) and Geideman (1932) attributed 
variegated fescue meadows to steppe communities, while Busch (1935) and Magakian (1941) regarded them as typical meadows. Later Jaroshenko (1942) suggested that Festucetum variae is a relict steppe, formed during the xerothermal period, and that since then, due to the further increase of climate humidity, the above-mentioned communities have been subject to the process of steppization. This opinion is shared by Grossheim (1948.Kimeridze $(1965 \mathrm{a}, \mathrm{b})$ considers them as meadows. Variegated fescue meadows are well-spread throughout the Caucasus. Though, in South Georgian Uplands, unlike the Great Caucasus, these communities occur on grazed areas on northern slopes.

Festuco variae - Caricetion meinshausianae / Festuco variae - Caricetum meinshausianae typicum (All. / Ass. nov.) Type relevé: N28

Main altitude: $2060-3050 \mathrm{~m}$

Character species: Primula amoena, Inula orientalis, Pyrethrum coccineum (Tanacetum coccineum), Gentiana septemfida, Oxytropis albana, Polygonum carneum, Minuartia circassica, Helictotrichon adzharicum, Seseli alpinum, Vicia alpestris

Very common in the Great Caucasus. Communities dominated by Polygonum carneum are of particular importance as natural grasslands. Under the influence of overgrazing, these meadows become replaced by more grazing resistant communities with robust tussock grasses.

Astrantia trifida variety Type relevé: N15

Character species: Astrantia trifida

Anthemio sosnovsyanae - Jurinellion subacaulis / Anthemio sosnovsyanae - Jurinellietum subacaulis typicum (All. / Ass. nov.) Type relevé: N1059

Character species: Anthemis sosnovskyana, Jurinella subacaulis, Aetheopappus caucasicus, Alchemilla sericea, Potentilla crantzii, Anemone speciosa, Campanula collina, Silene commutata, Geranium ruprechtii, Veronica telephïfolia, Alchemilla chlorosericea, Salix kazbekensis, Senecio taraxacifolius, Saxifraga flagellaris, Arenaria lychnidea, Saxifraga cartilaginea

Veronica telephüifoliae Alchemilletum chlorosericeae (Ass. nov.) Type relevé: N1069

Differential species: Veronica telephïfolia, Alchemilla chlorosericea

Rhinantho minoris- Trifolietalia pratensis (Ord. nov.) Type relevé: N401

Altitude: (1800)1850-2050-2200) m

Exposition: Never in East or Southwest exposition

Character species: Rhinanthus minor, Trifolium pratense, Festuca pratensis, Sisymbrium sp., Thlaspi sp.

Polygono alpestris - Taraxacion campylodes / Polygono alpestris - Taraxacetum campylodes typicum (All. / Ass. nov.) Type relevé: $\mathbf{N} 4012$

Main altitude: $1850-2050 \mathrm{~m}$

Character species: Polygonum alpestre, Taraxacum campylodes, Hieracium sp., Scutellaria leptostegia, Fumaria schleicheri, Phleum phleoides, Scrophularia sp., Scutellaria raddeana, Scutellaria raddeanus, Scutellaria sp., Silene lacera, Silene sp., Silene wallichiana, Bunias orientalis

Polygonum alpestre - Taraxacum campylodes variety Type relevé: N4013

Character species: Phleum phleoides, Scrophularia sp., Scutellaria raddeana, Scutellaria sp., Silene lacera, Silene sp., Silene wallichiana, Bunias orientalis

Differential species: Polygonum alpestre, Taraxacum campylodes, Hieracium sp., Scutellaria leptostegia, Fumaria schleicheri

Polygonum alpestre Taraxacum campylodes communities are found on wet gentle slopes, mostly within the range of the birch woodlands. Floristically, they are also connected with Rhododendron thickets.

Pedicularis chroorrhyncha - Tanacetum vulgare variety Type relevé: N4004

Character species: Pedicularis chroorrhyncha, Cerastium arvense, Bupleurum polyphyllum, Euphrasia hirtella, Anthyllis variegata, Phleum phleoides

Differential species: Tanacetum vulgare 
Alpine pastures: Sympoholomo graveolensis - Saxifragetea exaratae (Class. nov.) (=PAA TWINSPAN) Type relevé: $\mathbf{N 2 0 7 1}$

Altitude: 2800)2874-2920(3150) m Exposition: Mostly South

Character species: Symphyoloma graveolens, Saxifraga exarata, Taraxacum porphyranthum, Minuartia aizoides, Silene marcowiczii, Minuartia inamoena, Poa alpina, Potentilla crantzii

Alchemillo sericeae - Anthemetea sosnovskayae / Alchemilla sericeea Anthemietumn sosnovskayae typicum (Ord. / All. / Ass. nov.) (=MCC TWINSPAN) Type relevé: N2071

Main altitude: $2880-2920 \mathrm{~m}$

Character species: Alchemilla sericea, Anthemis sosnovskyana, Pedicularis chroorrhyncha, Myosotis alpestris, Campanula saxifraga, Minuartia inamoena, Crocus scharojanii, Taraxacum stevenii, Arenaria lychnidea, Minuartia oreina, Draba siliquosa, Veronica gentianoides

Arenaria lychnidea Minuartia aizoides variety Type relevé: N2050

Character species: Arenaria lychnidea, Minuartia aizoides, Pedicularis chroorrhyncha, Taraxacum stevenii

Arenaria serpyllifolia Minuartietum inamoenae variety Type relevé: N2066

Character species: Arenaria serpyllifolia, Minuartia inamoena, Taraxacum stevenii, Campanula collina, Minuartia oreina

Campanula saxifraga - Minuartieta aizoides variety Type relevé: $\mathbf{N 2 0 7 3}$

Main altitude: $2870-2920 \mathrm{~m}$

Character species: Campanula saxifraga, Minuartia aizoides

\section{Conclusions}

\section{Ethnobotany}

This study reported on 529 identified plant taxa from the subalpine and alpine regions of the Georgian Caucasus. Of these, based on our own data interviews as well as literature surveys, 183 were used in Georgia, mostly as fodder, food, and medicine. As many mountain regions all over the world, the rural areas of the Georgian Caucasus have suffered a constant population decline for decades, due to harsh economic conditions and lack of modern infrastructure. While this has especially accelerated the loss of traditional agricultural practices, it also has affected the use of wild gathered plants. It remains to be seen to what extent the border closures, and with them largely the cessation of cross border pasture use, will affect the use of traditional knowledge in the Georgian Caucasus in the future.

The process of genetic erosion of ancient crop varieties was originally of little concern for the mountain areas of Georgia, which until the 1990s acted as a depository of ancient crops. Nowadays the main reason for genetic erosion of ancient crop varieties is the demographic decline in mountain regions due to harsh economic conditions and lack of modern infrastructure (Nakhutsrishvili et al. 2009. The shift from ancient cultivars to modern highyielding crops such as maize and potato, which took place in the lowland areas much earlier, began in mountain villages after the end of Soviet occupation, when local inhabitants who had been forced to the lowlands, returned to their original villages. However, many villages in high altitude Georgia were abandoned under pressure during Soviet occupation, and while some families have returned at least for the summer, many villages were completely abandoned in the 1980s and remain in ruins. In occupied villages old household utensils like butter barrels are often to be found in storage, but not used anymore. Small bridges are still made from wood, but many other wooden household items like beautiful bed-headboards are simply discarded. Some implements, e.g., snowshoes or brooms are still maintained. Agricultural tools such as hay rakes are a common sight in abandoned barns, but more sought afar items like ox-drawn threshing sledges could only be found in museums. While sheep were produced on a large scale during Soviet times, leading to widespread overgrazing, nowadays only a few scattered herds remain, and traditional wool items are getting more difficult to find, while tourist products abound along roadsides especially in the outskirts of Tbilisi and resort areas like Borjomi and Barisako. Sadly, we could not find any grain cultivation anywhere at higher altitudes, although old landraces of wheat and barley were formerly preferred to prepare bread and beer for religious rituals. Many abandoned terrasses indicate where grain was formerly grown but now these fallow fields were long overgrown. No grain has been grown in the region for decades and residents indicated that last time barley was cropped 30 years ago 


\section{Vegetation}

The alpine vegetation of the Caucasus hotspot has fascinated botanists for centuries. Given the very complicated biogeographic setting, a concise classification of vegetation communities has however eluded science so far. All attempts to classify the alpine vegetation have suffered from the lack of sufficient releveés, given that many communities have been often described on very few, often only one, vegetation sample.

The present work, based on 619 plots, is the first study to attempt a concise phytosociological classification. However, even given this large number of samples a complete vegetation classification still proved difficult, and more releveés are needed for a detailed assessment. In the present study we employed traditional phytosociological table work combined with an analysis based on species composition, coverage and abiotic factors using "R", compared to a classification using "Twinspan". The results indicated that while Twinspan has been used for decades in phytosociology, the program failed to elucidate a detailed vegetation classification in the extremely diverse Caucasus ecosystem, albeit being useful for a coarse classification of higher vegetation units. This indicates that especially in very species rich communities a traditional classification is still preferable.

\section{Declarations}

Ethics statement: Prior to the survey, we obtained oral informed consent from each participant.

Consent to publish: This manuscript does not contain any personal data and does not require publishing permission.

Funding: The study was funded by the Saving Knowledge Foundation. The sponsor himself has no direct role in planning the research, collecting, and analyzing the data, or using the results.

Competition of interests: The authors state that they have no competing interests

Author contribution: GN and KB collected data and revised the first draft of the manuscript. RWB, IUR and REH analyzed and interpreted the data and results. RWB and IUR wrote the manuscript. RWB and MH revised the manuscript. All authors read and approved the final manuscript.

\section{Literature cited}

Adler DS, Bar-Oz G. 2009. Seasonal patterns of prey acquisition during the Middle and Upper Paleolithic of the southern Caucasus. In Hublin JJ, Richards M (eds) The Evolution of Hominid Diets: Integrating approaches to the study of Palaeolithic subsistence, Pp. 127-140. Springer, Leipzig, Germany.

Agakhanjanz OE, Breckle SW. 2002. Plant diversity and endemism in high mountains of Central Asia, the Caucasus and Siberia. In: Körner Ch, Spehn E (eds) Mountain biodiversity - a global assessment. Parthenon Publishing Group, Boca Raton, FL, pp 117-127.

Akhalkatsi M, Ekhvaia J, Asanidze Z. 2012. Diversity and Genetic Erosion of Ancient Crops and Wild Relatives of Agricultural Cultivars for Food: Implications for Nature Conservation in Georgia (Caucasus). In Tiefenbacher J. Perspectives on Nature Conservation - Patterns, Pressures and Prospects. InTech, Croatia.

Akhalkatsi M, Ekhvaia J, Mosulishvili M, Nakhutsrishvili G, Abdaladze O, Batsatsashvili K. 2010. Reasons and Processes Leading to the Erosion of Crop Genetic Diversity in Mountainous Regions of Georgia. Mountain Research and Development 30(3):304-310.

Akhalkatsi M. 2009. Conservation and Sustainable Use of Crop Wild Relatives in Samtskhe- Javakheti. Final Report. Elkana, Tbilisi, Georgia.

Altaf A, Haq SM, Shabnum N, Jan HA. 2021. Comparative assessment of Phyto diversity in Tangmarg Forest division in Kashmir Himalaya, India. Acta Ecologica Sinica.

Badr A, Müller K, Schäfer-Pregl R, El Rabey H, Effgen S, Ibrahim HH, Pozzi C, Rohde W, Salamini F. 2000. On the origin and domestication history of barley (Hordeum vulgare. Molecular Biology and Evolution 17(4):499-510.

Baselga A, Orme CDL. 2012. betapart: an R package for the study of beta diversity. Methods in Ecology and Evolution 3:808-812.

Baselga A, Orme D, Villeger S, De Bortoli J, Leprieur F, Logez M, Henriques-Silva R. 2018. Partitioning Beta Diversity into Turnover and Nestedness Components. Cran 1-42. 
Baselga A. 2010. Partitioning the turnover and nestedness components of beta diversity. Global Ecological Biogeography 19:134-143. doi: 10.1111/j.1466-8238.2009.00490.x

Baselga MA. 2013.Package 'betapart.'

Batsatsashvili K, Kikvidze Z, Bussmann RW (eds.) 2020a. Ethnobotany of Mountain Regions - Central Asia and Altai. Springer International Publishing, Cham. pp. XXII + 881, ISBN: 978-3-030-28946-1

Batsatsashvili K, Kikvidze Z, Bussmann RW (eds.) 2020b. Ethnobotany of Mountain Regions - Far Eastern Europe. Springer International Publishing, Cham.pp. XXIX + 1063, ISBN: 978-3-030-28939-3

Bedoshvili D. 2008. National Report on the State of Plant Genetic Resources for Food and Agriculture in Georgia. http://www.pgrfa.org/gpa/geo/Georgian report on State of PGR Sep 29, 2008.pdf.

Busch NA. 1935. Botanical and geographical studies of the Caucasus. Izd AN SSSR, Moscow/ Leningrad (in Russian).

Bussmann RW (ed.) 2017. Ethnobotany of the Caucasus. Springer International Publishing International Publishing: Cham, XXVII, 746p. (ISBN 978-3-319-49411-1)

Bussmann RW, Paniagua Zambrana NY, Sikharulidze S, Kikvidze Z, Darchidze M, Manvelidze Z, Ekhvaia J, Kikodze D, Tchelidze D, Khutsishvili M, Batsatsashvili K, Hart RE. 2020a. Form the sea to the mountains - plant use in Ajara, Samegrelo and Kvemo Svaneti, Sakartvelo (Republic of Georgia), Caucasus. Ethnobotany Research and Applications 20(9) doi: 10.32859/era.20.9.1-34

Bussmann RW, Paniagua Zambrana NY, Sikharulidze S, Kikvidze Z, Kikodze D, Jinjikhadze T, Shanshiashvili T, Chelidze D, Batsatsashvili K, Bakanidze N. 2014. Wine, Beer, Snuff, Medicine and loss of diversity - Ethnobotanical travels in the Georgian Caucasus. Ethnobotany Research and Application, 12: 237-313.

Bussmann RW, Paniagua Zambrana NY, Sikharulidze S, Kikvidze Z, Kikodze D, Tchelidze D, Batsatsashvili K, Hart RE. 2017d. Plant and fungal use in Tusheti, Khevsureti and Pshavi, Sakartvelo (Republic of Georgia), Caucasus. Acta Societatis Botanicorum Poloniae 86(2):3517. doi: 10.5586/asbp.3517.

Bussmann RW, Paniagua Zambrana NY, Sikharulidze S, Kikvidze Z, Kikodze D, Tchelidze D, Batsatsashvili K, Hart RE. 2021. An ethnobotany of Upper Imereti, Ukana Pshavi, Meshketi and Pankisi gorge, Sakartvelo (Republic of Georgia), Caucasus. Bocconea 29:33-54. doi: 10.7320/10.7320/Bocc29.001

Bussmann RW, Paniagua Zambrana NY, Sikharulidze S, Kikvidze Z, Kikodze D, Tchelidze D, Khutsishvili M, Batsatsashvili K, Hart R E. 2020b. An ethbobotany of Kahkheti and Kvemo Kartli, Sakartvelo (Republic of Georgia), Caucasus. Ethnobotany Research and Applications 19(35) doi: 10.32859/era.19.47.1-28

Bussmann RW, Paniagua Zambrana NY, Sikharulidze S, Kikvidze Z, Kikodze D, Tchelidze D, Batsatsashvili K, Hart RE. 2018. Unequal brothers - Plant and fungal use in Guria and Racha, Sakartvelo (Republic of Georgia), Caucasus. Indian Journal of Traditional Knowledge 17(1):7-33.

Bussmann RW, Paniagua Zambrana NY, Sikharulidze S, Kikvidze Z, Kikodze D, Tchelidze D, Batsatsashvili K, Hart RE. 2017b. Plants in the spa - the medicinal plant market of Borjomi, Sakartvelo (Republic of Georgia), Caucasus. Indian Journal of Traditional Knowledge 16(1), 25-34.

Bussmann RW, Paniagua Zambrana NY, Sikharulidze S, Kikvidze Z, Kikodze D, Tchelidze D, Batsatsashvili K, Hart RE. 2017a. Ethnobotany of Samtskhe-Javakheti, Sakartvelo (Republic of Georgia), Caucasus. Indian Journal of Traditional Knowledge 16(1), 7-24.

Bussmann RW, Paniagua Zambrana NY, Sikharulidze S, Kikvidze Z, Kikodze D, Tchelidze D, Khutsishvili M, Batsatsashvili K, Hart RE, Pieroni A. 2016c. Your poison in my pie - the use of Potato (Solanum tuberosum L.) leaves in Sakartvelo, Georgia (Caucasus) and Gollobordo, Eastern Albania. Economic Botany 70(4), 431-437.

Bussmann RW, Paniagua Zambrana NY, Sikharulidze S, Kikvidze Z, Kikodze D, Tchelidze D, Khutsishvili M, Batsatsashvili K, Hart RE. 2016b. A comparative ethnobotany of Khevsureti, Samtskhe-Javakheti, Tusheti, Svaneti, and Racha-Lechkhumi, Republic of Georgia (Sakartvelo), Caucasus. Journal of Ehnobiology and Ethnomedicine 12:43, DOI: 10.1186/s13002-016-0110-2. 
Bussmann RW, Paniagua Zambrana NY, Sikharulidze S, Kikvidze Z, Kikodze D, Tchelidze D, Khutsishvili M, Batsatsashvili K, Hart RE. 2016a. Medicinal and food plants of Svaneti and Lechkhumi, Sakartvelo (Republic of Georgia), Caucasus. Medicinal and Aromatic Plants 5:266. doi: 10.4172/2167-0412.1000266.

Bussmann RW, Paniagua Zambrana NY, Sikharulidze S, Kikvidze Z, Kikodze D, Tchelidze D, Batsatsashvili K, Hart RE. 2017c. Ethnobotany of Samtskhe-Javakheti, Sakartvelo (Republic of Georgia), Caucasus. Indian Journal of Traditional Knowledge 16(1):7-24.

Bussmann RW, Paniagua Zambrana NY, Sikharulidze S, Kikvidze Z, Kikodze D, Tchelidze D, Batsatsashvili K, Hart RE. 2016c. Medicinal and food plants of Svaneti and Lechkhumi, Sakartvelo (Republic of Georgia), Caucasus. Medicinal and Aromatic Plants 5:266. doi: 10.4172/2167-0412.1000266.

Bussmann RW, Paniagua Zambrana NY, Sikharulidze S, Kikvidze Z, Kikodze D, Tchelidze D, Batsatsashvili K, Hart RE.

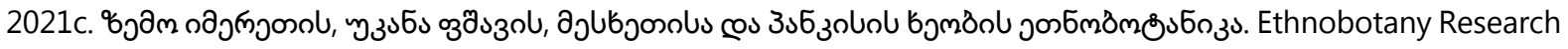
and Applications 22:15. doi: 10.32859/era.22.15.1-22

Bussmann RW, Paniagua Zambrana NY, Sikharulidze S, Kikvidze Z, Kikodze D, Tchelidze D, Batsatsashvili K, Hart RE.

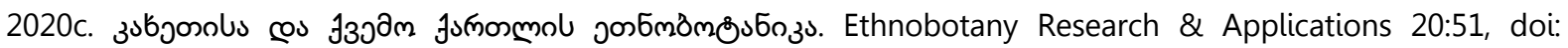
10.32859/era.20.51.1-23

Bussmann RW, Paniagua Zambrana NY, Sikharulidze S, Kikvidze Z, Kikodze D, Tchelidze D, Batsatsashvili K, Hart RE.

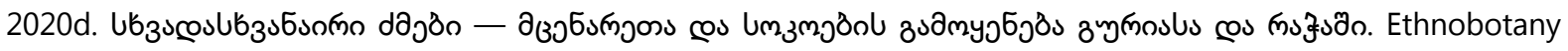
Research \& Applications 20:52, doi: 10.32859/era.20.55.1-34

Bussmann RW, Paniagua Zambrana NY, Sikharulidze S, Kikvidze Z, Kikodze D, Tchelidze D, Batsatsashvili K, Hart RE.

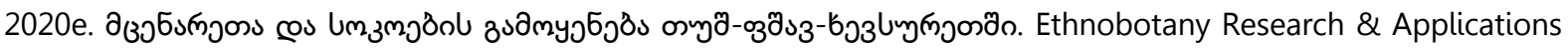
20:53, doi: 10.32859/era.20.53.1-30

Bussmann RW, Paniagua Zambrana NY, Sikharulidze S, Kikvidze Z, Kikodze D, Tchelidze D, Khutsishvili M,

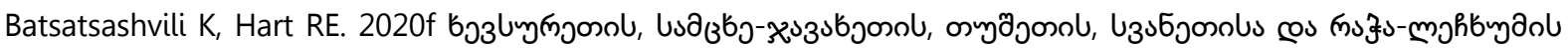

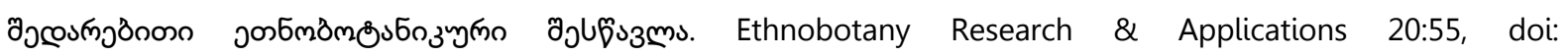
10.32859/era.20.55.1-30

Bussmann RW, Paniagua Zambrana NY, Sikharulidze S, Kikvidze Z, Kikodze D, Tchelidze D, Batsatsashvili K, Hart RE.

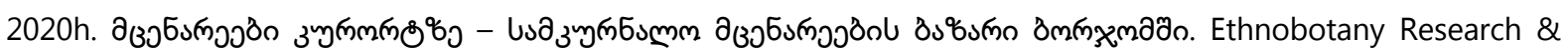
Applications 20:56, doi: 10.32859/era.20.56.1-23

Bussmann RW, Paniagua Zambrana NY, Sikharulidze S, Kikvidze Z, Kikodze D, Tchelidze D, Khutsishvili M,

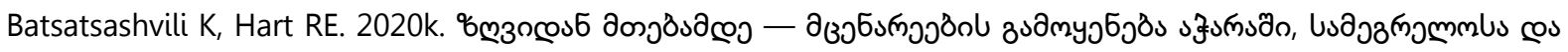

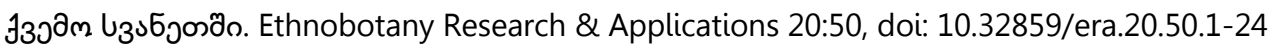

Bussmann RW, Paniagua Zambrana NY, Ur Rahman I, Kikvidze Z, Sikharulidze S, Kikodze D, Tchelidze D, Khutsishvili M, Batsatsashvili K. 2021b. Unity in diversity - Food plants and fungi of Sakartvelo (Republic of Georgia), Caucasus. Journal of Ethnobiology and Ethnomedicine.

Bussmann, R.W., Paniagua Zambrana, N.Y., Sikharulidze, S., Kikvidze, Z., Kikodze, D., Tchelidze, D., Batsatsashvili, K.,

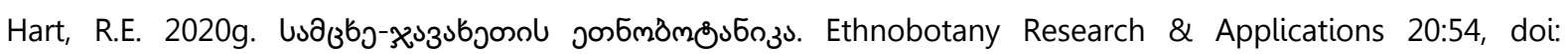
10.32859/era.20.54.1-22

Dekaprelevich L, Menabde V. 1929. Kizucheniu polevykh kultur zapadnoi Gruzii. I. Racha. (Study of cereal cultivars in Georgia. I. Racha. Scientific Papers of the Applied Sections of the Tbilisi Botanical Garden 62):219-252. (In Russian).

Dolukhanov AG. 1946. Upper limits of alpine vegetation in the headwaters of Avar Koy-Su (Dagestan.Trudy Tbilisis Botanikuri Institutshi 9:131-154 (in Russian).

Dolukhanov AG. 2010. Forest vegetation of Georgia. Universal, Tbilisi (in Russian). 
Elenevskiy AG. 1964. For the question of origin of the alpine flora of the Lesser Caucasus. Bulletin Moskwa ObshchVa Isp Prirody Biological Section V 69(6):67-77 (in Russian).

Elenevskyi AG. 1965. Flora of Zangezur and some questions of history of the flora of Transcaucasus. Kandidatskaya dissertatsia (in Russian).

Ellenberg H. 1996. Vegetation Mitteleuropas mit den Alpen. Ulmer, Stuttgart

Englisch T. 1993. Salicetea herbacea. In: Grabherr G, Mucina L (eds) Die Pflanzengesellschaften Osterreichs. Gustav Fischer, Jena, pp 382-401.

Fedorov AA. 1942. Alpine carpets of the Caucasus and their origin. Bulletin Akademia Nauk Armenia SSR 4:137-155 (in Russian).

Fedorov AA. 1952. The history of high mountain flora of the Caucasus in the Quatemary period as an example of autochthonous development of the Tertiary floristic basis. In: Kristophovich AN. (ed) Materialy po chetverlichnomu periodu. SSSR, 3, Moscow, pp 49-86 (in Russian)

Feodorov FF. 1952. The history of high mountain flora of the Caucasus in the quaternary period as an example of autochthonous development of the Tertiary floristic basis. In: Kristophovich AN (ed) Materialy po chetvertichnomu periodu. SSSR, 3, Moscow, pp 49-86.

Finlayson C. 2005. Biogeography and evolution of the genus Homo. Trends in Ecology and Evolution 20(8):457463.

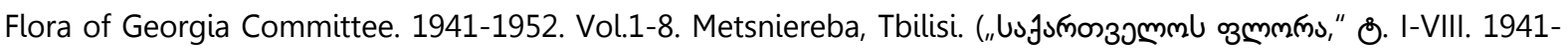
1952).

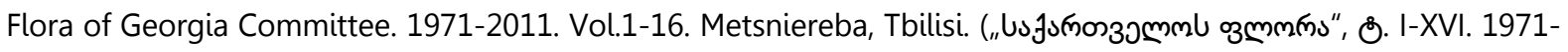
2011).

Fox J, Weisberg S. 2018. An R companion to applied regression. Sage Publications, California.

Gabunia L, Vekua A, Swisher CC, Ferring R, Justus A, Nioradze M, Ponce de Leon M, Tappen M, Tvalchrelidze M, Zollikofer C. 2000. Earliest Pleistocene hominid cranial remains from Dmanisi, Republic of Georgia: taxonomy, geological setting, and age. Science 288(5578):85-89.

Gabunia L, Vekua AA. 1995. Plio-Pleistocene hominid from Dmanisi, East Georgia, Caucasus. Nature 373(6514):509-512.

Gagnidze R. 2005. Vascular plants of Georgia: a nomenclatural checklist. Tbilisi, Ketskhveli Inst Bot, Javakhishvili

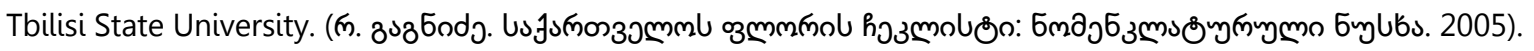

Gagnidze RI. 2000. Diversity of Georgia's Flora. In: Berutchashvili N, Kushlin A, Zazanashvili N (eds) Biological and landscape diversity of Georgia. WWF Georgia Country Office, Tbilisi, pp 21-33.

Geideman TS. 1932. The studies on vegetation of high mountain pastures of Kurdistan. Trudy po geobot obsledov pastbish SSR Azerbaidzhana, Publishing House of the Academy of Sciences of Azerbaijan SSR, Baku, ser. B, 5 (in Russian).

Greig-Smith P. 1983. Quantitative Plant Ecology, 3rd ed. University of California Press.

Grossheim AA. 1936. The analysis of Caucasian flora. Trudy Botanikuri Institutshi Azerbaijan AN SSSR lzd Az Fil Armenian SSSR. Baku (in Russian).

Grossheim AA. 1948. Vegetation cover of the Caucasus. MOIP, Moscow (in Russian).

Gulisashvili V. 1964. Natural zones and natural history areas of the Caucasus. Nauka, Moscow, p 326 (in Russian).

Güner A. 2012. Turkey plant list. ANG Foundation, Istanbul.

Haq F, Ahmad H, Iqbal Z, Alam M, Aksoy A. 2017.Multivariate approach to the classification and ordination of the 
forest ecosystem of Nandiar valley western Himalayas. Ecological Indicators 80:232-241. doi: 10.1016/j.ecolind.2017.05.047

Haq SM, Calixto ES, Kumar M. 2021a. Assessing biodiversity and productivity over a small-scale gradient in the protected forests of Indian Western Himalayas. Journal of Sustainable Forestry, 40(7), pp.675-694.

Haq SM, Calixto ES, Rashid I, Khuroo AA. 2021b. Human-driven disturbances change the vegetation characteristics of temperate forest stands: A case study from Pir Panchal mountain range in Kashmir Himalaya. Trees, Forests and People, 6, p.100134.

Haq SM, Yaqoob U, Calixto ES, Rahman IU, Hashem A, Abd_Allah EF, Alakeel MA, Alqarawi AA, Abdalla M, Hassan M, Bussmann RW. 2021. Plant Resources Utilization among Different Ethnic Groups of Ladakh in Trans-Himalayan Region. Biology, 10(9), p.827.

Hassan M, Yaqoob U, Haq M, Lone FA, Habib H, Hamid S, Jan HA, Bussmann RW. 2021. Food and culture: Cultural patterns related to food by indigenous communities in Kashmir-A Western Himalayan region. Ethnobotany Research and Applications 22:1-20.

Hervé M. 2020. RV Aide Memoire: Testing and Plotting Procedures for Biostatistics.

Hill MO. 1979. TWINSPAN: a FORTRAN program for arranging multivariate data in an ordered two-way table by classification of the individuals and attributes. Cornell Univ.

ljaz F, Rahman I, Iqbal Z, Alam J, Ali N, Khan S. 2018. Ethno-ecology of the healing forests of Sarban Hills, Abbottabad, Pakistan: an economic and medicinal appraisal, in: Ozturk M. (ed.) Plant and Human Health. Springer International Publishing AG, pp. 675-706. doi: 10.1007/978-3-319-93997-1_19

ljaz F. 2014. Biodiversity and traditional uses of plants of Sarban Hills, Abbottabad. M.Phil Dissertation, Hazara University Manehra-21300, Khyber Pakhtunkhwa, Pakistan.

Janelidze C, Margalitadze N. 1977. History of Forest Vegetation in Holocene. In: Nakhutsrishvili G. (ed) Alpine Ecosystems of Kazbegi. Publishing House of the Georgian Academy of Sciences, Tbilisi, pp 17-21.

Jaroshenko PD. 1942. On vegetation changes in the forested region of the Trans-Caucasus. Izd Armyansk Fil AN SSSR, Erevan 721):32-46 (in Russian).

Javakhishvili I. 1987. Sakartvelos ekonomiuri istoria (Economic History of Georgia), (Ed. 2), Vol.5., Metsniereba, Tbilisi, Georgia. (In Georgian).

Kamelin RV. 2001. The genus Sibbaldia L. In: Tsvelev, NN (ed) Flora of Eastern Europe. St. Petersburg: Mir i semja, Publishing House of St. Petersburg State Chemical Pharmaceutical Academy 10:456-457 (in Russian).

Ketskhoveli N, Kharadze A, Gagnidze R (eds.1971-2011. Flora of Georgia, 2nd ed, vols I-XVI, Metsniereba, Tbilisi (in Georgian).

Ketskhoveli N, Ramishvili M, Tabidze D. 1960. Sakartvelos ampelograpia. (Amphelography of Georgia.Georgian Academy of Sciences Press, Tbilisi, Georgia. (In Georgian).

Ketskhoveli N. 1928. Masalebi kulturul mtsenareta zonalobis shesastsavlad kavkasionze. (Materials on zonal distribution of cultivated plants in the Greater Caucasus.Agricultural National Committee Press, Tbilisi, Georgia. (In Georgian).

Ketskhoveli N. 1957. Kulturul mtsenareta zonebi sakartveloshi (Zones of cultivated plants in Georgia.Georgian Academy of Sciences Press, Tbilisi, Georgia. (In Georgian).

Kharadze A. 1944. Description of Upper Svaneti subnival belt flora. Notulae systematicae ac geographicae Instituti Botanici Thbilissiensis 12. Tbilisi (in Russian).

Kharadze A. 1948a. For study of xerophilous floras of the Rocky range. Works of the Tbilisi Institute of Botany 12.

Kharadze AL. 1960. An endemic hemixerophilous element of Greater Caucasus uplands. Problems in Botany 5:115126. 
Kharkevich SS. 1954. Role of epeirogenesis of the Quaternary in the formation of the high mountain flora of the Greater Caucasus. Botanijesky Zhurnal 39(4):498-514 (in Russian).

Kimeridze KR. 1965a. For geobotanical study of alpine sedge wetlands on the Great Caucasus. Metsniereba, Tbilisi (in Georgian).

Kimeridze KR. 1965b. Materials for the Flora of wetland vegetation of the Central Great Caucasus. Metsniereba, Tbilisi (in Georgian).

Kolakovsky AA. 1961. Plant world of Colchis. Publishing House of Moscow University, Moscow (in Russian).

Körner C, Paulsen J, Spehn EM. 2011. A definition of mountains and their bioclimatic belts for global comparisons of biodiversity data. Alpine Botany 121:73-78.

Körner C, Paulsen J. 2004. A world-wide study of high-altitude treeline temperatures. Journal of Biogeography 31:713-732.

Körner C. 2003. Alpine plant life. Functional plant ecology of high mountain ecosystems. Springer, Berlin.

Körner C. 2004. Mountain biodiversity, its causes and function. Ambio Special Report 13:11-17.

Kürschner H, Parolly G, Raab-Straube E. 1998. Phytosociological Studies on high mountain plant communities of the Taurus mountains (Turkey) 3. Snow-patch and meltwater communities. Feddes Repertorium 109(7-8):581-616.

Kuznetsov NI. 1915. Short survey of history of vegetation development. Bulletin of the Russian Flora 1(1):1-16.

Larcher W. 1980. Klimastress im Gebirge: Adaptationstraining und Selektionsfilter für Pflanzen. Rheinisch-Westfal Akad Wiss Leverkusen: Westdeutscher Verlag 291:49-79.

Lenth R. 2020. EMMEANS: Estimated Marginal Means, aka Least-Squares Means.

Lou Y, Pan Y, Gao C, Jiang M, Lu X, Xu YJ. 2016. Response of plant height, species richness and aboveground biomass to flooding gradient along vegetation zones in floodplain wetlands, Northeast China. PLoS One 11:e0153972.

Löve D. 1970 Subarctic and subalpine: where and what? Arctic and Alpine Researtch 2:63-73.

Magakian AK. 1941. Vegetation of Armenian SSR. Izd AN SSSR, Leningrad (in Russian)

Maleev WP. 1948. Main stages of vegetation development of Mediterranean and southern mountain areas of USSR (the Caucasus and Crimea) in Querternary. Works Nikitsky Botanical Garden 25(1-2):3-28.

Malhi Y, Franklin J, Seddon N, Solan M, Turner MG, Field CB, Knowlton N. 2020. Climate change and ecosystems: Threats, opportunities and solutions. Philosophical Transactions of the Royal Society B, 375(1794), p.20190104.

Mayor JR, Sanders NJ, Classen AT, Bardgett RD, Clément JC, Fajardo A, Lavorel S, Sundqvist MK, Bah M, Chisholm C, Cieraad E, Gedalof Z, Grigulis K, Kudo G, Oberski DL, Wardle, D.A. 2017. Elevation alters ecosystem properties across temperate treelines globally. Nature 542:91-95. doi: 10.1038/nature21027

McGovern, P.E. 2003. Ancient wine: the search for the origins of viniculture. Princeton University Press, Princeton, New Jersey, USA.

Medvedev JS. 1915. Vegetation of the Caucasus. Works Tiflis Botanical Garden 1(18):1-88.

Melikishvili G. (Ed.) 1970. Sakartvelos istoriis narkvevebi (Historical essays of Georgia.Tbilisi, Georgia. (In Georgian).

Menabde V. 1938. Sakartvelos kerebi (Barleys of Georgia). Georgian Academy of Sciences Press, Tbilisi, Georgia. (In Georgian).

Menabde V. 1948. Pshenitsi Gruzii (Wheats of Georgia). Georgian Academy of Sciences Press, Tbilisi, Georgia. (In Russian).

Mikeladze RM. 1960. About the origin of the snowbed communities of the Caucasus. In: Proceedings of scientific works of young scientists. Academy of Science of the Georgian SSR, Tbilisi, p 33-41 (in Russian). 
Mir AY, Yaqoob U, Hassan M, Bashir F, Zanit SB, Haq SM, Bussmann RW. 2021. Ethnopharmacology and phenology of highaltitude medicinal plants in Kashmir, Northern Himalaya. Ethnobotany Research and Applications, 22:1-15.

Nakhutsrishvili G, Abdaladze O, Batysatsashvili K, Spehn E. 2017. Plant Diversity in the Central Great Caucasus. Geobotany Studies, doi 10.1007/978-3-319-55777-9_2. Springer, Heidelberg and Berlin.

Nakhutsrishvili G, Akhalkatsi M, Abdaladze O. 2009. Main threats to the mountain biodiversity in Georgia (the Caucasus.Mountain Forum Bulletin 92):18-19.

Nakhutsrishvili G, Zazanashvili N, Batsatsashvili K. 2011. Why the Colchis and Hyrcan relict forests of the western Eurasian Caucasus should be considered "temperate rainforest". - In: Della Sala D. (ed.) Temperate and Boreal Rainforests of the World: Ecology and Conservation. Island Press, Wadhington DC, Pp. 214-221.

Nakhutsrishvili G. 1999. The vegetation of Georgia (Caucasus). Braun-Blanquetia 15:1-74.

Nakhutsrishvili G. 2013. The Vegetation of Georgia (South Caucasus), Geobotany Studies. doi:10.1007/978-3-64229915-5_8, Springer-Verlag, Berlin and Heidelberg.

Narinyan SG. 1959. Preliminary results of ecological study of alpine snowbeds in Aragats. Bulletin of the Academy of Science of the Armenian SSR (Biological Sciences) 12:3-15 (in Russian).

Oksanen J, Blanchet, Friendly M, Kindt R, Legendre P, Minchin D, Minchin P, O'Hara R, Simpson G, Solymos P, Stevens M, Szoecs E, Wagner H. 2019. vegan: Community Ecology Package (R package Version 2.5-5).

Onipchenko VG. 2004. Alpine ecosysteme in the Nordwest Caucasus. Kluwer Academic, Berlin (in Russian)

Pauli H, Gottfried M, Dullinger S, Abdaladze O, Akhalkatsi M, Benito Alonso JL, Coldea G, Dick J, Erschbamer B, Calzado RF, Ghosn D, Holten JI, Kanka R, Kazakis G, Kolla'r J, Larsson P, Moiseev P, Moiseev D, Molau U, Mesa JM, Nagy L, Pelino G, Puscas, M, Rossi G, Stanisci A, Syverhuset AO, Theurillat J-P, Tomaselli M, Unterluggauer P, Villar L, Vittoz P, Grabherr G. 2012. Recent plant diversity changes on Europe's mountain summits. Science 336(6070):353355

Pauli H, Gottried M, Grabherr G. 2011. Nemorele und meditterane Hochgebirge: Klima, Vegetationstufen, Artenfielfalt und Klimawandel am Beispiel der Alpen und Spanischen Sierra. In Nevada, Anhuf D, Fickert T and Gruninger F (eds) 2011. Ökozonen im Wandel. Selbstverlag Fach Geographie der Universitat Passau, Passau, pp 145-158.

Payal K, Rao KS, Maikhuri RK, Kandari LS. 2020. Ethnobotanical assessment of endangered Allium stracheyi (Baker): a lesser known species of the Nanda Devi Biosphere Reserve, Central Himalaya, India. In Ethnomedicinal plant use and practice in traditional medicine (pp. 1-16). IGI Global.

Pistrick K, Akhalkatsi M, Girgvliani T, Shanshiashvili T. 2009. Collecting plant genetic resources in Upper Svaneti (Georgia, Caucasus Mountains). Journal of Agriculture and Rural Development in the Tropics and Subtropics, Supplement 92: 127-135.

Popov MG. 1949. Description of the vegetation and flora of the Carpathians. Materials for study of flora and fauna of the USSR, new ser. of botany section. Moscow: Mosk. obshch-vo isp. prirpdy, issue 5 (13) (in Russian).

Pysek P, Srütek M. 1989. Numerical phytosociology of the subalpine belt of the Kazbegi region, Caucasus, USSR. Vegetatio 81:199-208.

R Core Team. 2020. R: A language and environment for statistical computing. R Foundation for Statistical Computing, Vienna, Austria.

Rahman IU, Afzal A, Iqbal Z, Alzain MN, Al-Arjani ABF, Alqarawi AA, Abd_Allah EF, Ali N, Sakhi S, Khan MA, Khan U. 2022. Classification and Characterization of the Manoor Valley's (Lesser Himalaya) Vegetation from the SubtropicalTemperate Ecotonal Forests to the Alpine Pastures along Ecological Variables. Plants, 11(1), p.87.

Rahman IU, Afzal A, Iqbal Z, Bussmann RW, Alsamadany H, Calixto ES, Shah GM, Kausar R, Shah M, Ali N, ljaz F. 2020. Ecological gradients hosting plant communities in Himalayan subalpine pastures: Application of multivariate approaches to identify indicator species. Ecological Informatics 60:101162. doi:10.1016/j.ecoinf.2020.101162 
Ramishvili R. 1988. Dikorastushii vinograd Zakavkazia (Wild Grape of the South Caucasus) (in Russian.Ganatleba, Tbilisi, Georgia.

Sakhokia M, Khutsishvili E. 1975. Synopsis of the flora of higher plants of Khevi. Metsniereba, Tbilisi (in Russian).

Schatz G, Shulkina T, Nakhutsrishvili G, Batsatsashvili K, Tamanyan K, Alizade V, Kikodze D, Geltman D, Ekim T. 2009. Development of Plant Red List Assessments for the Caucasus Biodiversity Hotspot. In Zazanashvili N, Mallon D (eds) Status and Protection of Globally Threatened Species in the Caucasus Pp. 188-192. Contour Ltd., USA.

Schatz G, Shulkina T, Solomon J. (eds) 2014. Red list of the endemic plants of the Caucasus region: Armenia, Azerbaijan, Georgia, Iran, Russia, Turkey. Missouri Botanical Garden Press, Saint Louis, MO.

Shatilova I, Mchedlishvili N, Rukhadze L, Kvavadze E. 2011. The history of the flora and vegetation of Georgia (South Caucasus.Publishing House of the Academy of Sciences of Georgia, Tbilisi.

Smilauer P, Jan L. 2014. Multivariate analysis of ecological data using CANOCO, 2nd ed. Cambridge University Press, Cambridge CB2 8BS, United Kingdom. doi: 10.1017/CBO9781139627061

Son HJ, Park SH, Lee DH, Song JH, Park HW, Park WG. 2019. Growing environment characteristics and vegetation structure of Vaccinium oldhamii Miq. native habitats in Korea. Forest Science and Technology 15:33-43.

Song JH, Cheong EJ, Kim H, Kim MS, Kim SH. 2015. Variation of morphological characteristics and anthocyanin contents from fruit of Vaccinium oldhamii in Korea. Journal of the Korean Society for Forest Science 104:193-197.

Takahashi K. 2018. Virtual issue: Alpine and subalpine plant communities: importance of plant growth, reproduction and community assemblage processes for changing environments. Journal of plant research, 131(6), pp.891-894.

Takhtajan AL. 1946. On the history of vegetation development in Armenia. Trudy Botanikuri Institutshi Akademia Nauk Armenii, Erevan 4:51-107 (in Russian).

Takhtajan AL. 1957. For the question of the origin of temperate flora of Eurasia. Botanijeski Zhurnal 42 (11):16351653.

Terzi M, Bogdanović S, D'Amico FS, Jasprica N. 2019. Rare plant communities of the Vis Archipelago (Croatia.Botanical Letters 1-14.

This P, Lacombe T, Thomas MR. 2006. Historical origins and genetic diversity of wine grapes. Trends in Genetics 22(9):511-519.

Tian Z, Li H, Li W, Gan X, Zhang X, Fan Z. 2018. Structural characteristics and niches of dominant tree populations in Tetracentron sinense communities: implications for conservation. Botanical Science 96:157-167.

Tumadjanov II. 1947. Forest vegetation of Teberda valley in the light of post-glacial history of phytolandscapes development. Trudy Tbilisis Botanikuris instituta 11:98-104 (in Russian).

Yang X, Li Y, Niu B, Chen Q, Hu Y, Yang Y, Song L, Wang J. Zhang G. 2021. Temperature and Precipitation Drive Elevational Patterns of Microbial Beta Diversity in Alpine Grasslands. Microbial ecology, pp.1-13.

Zernov AS. 2006. Flora of North-Western Caucasus. Tovarishchestvo nauchnikh izdanii, Moscow.

Zhizhizlashvili K, Berishvili T. 1980. Zemo Svanetis kulturul mtsenareta shestsavlisatvis (Study of cultivated plants in Upper Svaneti). Bulletin of Georgian Academy of Sciences 1002):417-419. (In Georgian). 